\title{
CHARACTERS OF CONNECTED LIE GROUPS ${ }^{1}$
}

\author{
BY \\ L. PUKANSZKY \\ University of Pennsylvania, Philadelphia, Pa. 19104, USA
}

\section{Introduction}

Let $G$ be a finite group and $(S)$ the group algebra of $G$ over the complex field. If $U$ is a unitary representation of $G$ on a finite dimensional unitary space $H, U$ extends to a unique * homomorphism $U^{\prime}$ of $(S)$ into the full operator algebra of $H$, and conversely. $U$ is multiple of an irreducible representation (or $U$ is a factor representation) if and only if the kernel of $U^{\prime}$ is a (two-sided) prime ideal of (B). If $U$ and $V$ give rise to the same prime ideal, they are multiples of the same irreducible representation, in which case we call them quasi-equivalent. We denote by $\operatorname{Prim}(G)$ the set of all prime ideals of $\mathscr{G}$ and by $\hat{G}$ the family of all quasi-equivalence classes of factor representations of $G$. Summing up, there is a canonical bijection between any two of the following three sets: Prim $(G), \hat{G}$ and the set of all characters of $G$.

Let now $G$ be a separable locally compact group. The theory of characters of such groups was initiated by R. Godement (cf. [11], [12], [13]). One major outgrowth of his investigations was the recognition of the fact that, in order that one should be able to associate with a (in general now infinite dimensional) continuous unitary representation of $G$ a character, beside generating a factor in the sense of F. J. Murray and J. v. Neumann, it must carry a special property, to be called normalcy in the sequel. In particular for this, in general, irreducibility is neither necessary nor sufficient. The notion of character inspired by Godement's work was formalized in the language of $C^{*}$ algebras by A. Guichar$\operatorname{det}$ (cf. [14], and [4], $\S 17$, p. 305). We recall (cf. [4], 13.9, p. 270), that we can associate with $G$ a $C^{*}$ algebra $C^{*}(G)$ (to be denoted in the following by (G) such that there is a canonical bijection between continuous unitary representations of $G$ and nondegenerate * representations of $\mathbb{S}$. This being so, we call a unitary representation $U$ normal, if (1) The ring of operators ( $v$. Neumann algebra) $M$ generated by $U$ is a semifinite factor, (2) If $\Phi$

(1) This work was supported by a grant from the National Science Foundation. 6-742901 Acta mathematica 133. Imprimé le 3 Octobre 1974 
is a faithful, normal, semifinite trace on $\mathbf{M}^{+}$, there is an element $v$ in $\mathcal{S S}^{+}$such that $\Phi(U(v))$ is positive and finite. The character belonging to $U$ is then the function $v \mapsto \Phi(U(v))$ $(\leqslant+\infty)$ on $\mathfrak{B S}^{+}$. We could first define analogously characters of an arbitrary $C^{*}$ algebra (cf. [4], 6.7, p. 120) and call characters of $G$ those of $(S)$ (cf. [14], 17.1.1, p. 305). Quasiequivalent normal representations give rise to proportional characters and conversely (cf. [4], 6.7.4, Corollaire, p. 127). Let $\hat{G}$ be the set of all quasi-equivalence classes of factor representations of $G$ (cf. e.g. [4], 18.6.2, p. 323) and $\hat{G}_{\text {norm }}$ the subset of $\hat{G}$ corresponding to normal representations. By what we have just said, there is a canonical bijection between $\hat{G}_{\text {norm }}$ and the set of all characters of $G$.

It follows from results by J. Glimm (cf [4], $\S 9$, p. 168), that we have $\hat{G}_{\text {norm }}=\hat{G}$, if and only if $G$ is of type I, that is, if any of its factor representations is multiple of an irreducible representation. In this case there is a canonical identification between the dual $\hat{G}$ (= set of unitary equivalence classes of irreducible unitary representations) of $G$ and $\hat{G}$, and conversely. Let us add, that if $G$ is not of type I, again by virtue of a theorem of Glimm (cf. e.g. [4], 9.5.6, p. 185) $\hat{G}$ does not lend itself to an effective parametrization, and seems to claim no interest.

Let now $G$ be a connected Lie group. It is known, that in important special cases, e.g. if $G$ is semi-simple or nilpotent, $G$ is also of type $I$. Lacking special assumptions, however, it fails to be such already in five dimensions. If $G$ satisfies additional conditions, for instance, if it is unimodular or solvable, by extension of the classical argument of Peter and Weyl one can show the existence of a separating family of normal representations. But not even here have been so far general statements, characterizing the size of $\hat{G}_{\text {norm }}$, available.

The principal result of the present paper (cf. Theorem 1, section IV) is an existence theorem for characters which, by aid of $C^{*}$ algebras, we can state in close analogy with the situation offerred by finite groups. We recall, that a closed, two-sided ideal of $\left(\mathfrak{S}=C^{*}(G)\right.$ is called primitive, if it is kernel of an irreducible representation. By results due to J. Dixmier (cf. [3], p. 100) (1) Any closed two-sided prime ideal of (SS is primitive and conversely, (2) The kernel of any factor representation is primitive. Let Prim $(G)$ be the set of all such ideals of $(S)$. There is an evident surjection $\delta$ from $\hat{G}$ onto Prim $(G)$, assigning to $l$ in $\hat{G}$ the kernel of any factor representation of class $l$. This being said we show, that the restriction $\eta$ of $\delta$ to $\hat{G}_{\text {norm }}$ is a bijection with Prim $(G)$. In other words, by what we said above, we are led to a canonical bijection between the set of all closed two-sided prime ideals of $B S$ and that of all characters of $G$ resp.-Let us note, that by virtue of an example due to Guichardet (cf. [14], Proposition 2, p. 62) the analogous statement for an arbitrary locally compact separable group would be false--An easy byproduct of our proof is the exi- 
stence of a separating family of normal representations for any connected Lie group (cf. Corollary to Theorem 1).

Let us assume next, that $G$ is simply connected and solvable. Not long ago L. Auslander and B. Kostant gave a necessary and sufficient condition in order that $G$ be of type I. For this case they provided an explicite description, involving orbits of the coadjoint representation, of $\hat{G}$ (cf. for all this [1]). The starting point of our present investigations was an attempt to extend to an arbitrary solvable group some of their constructions. This led us to associate with any such group a family $\subseteq$ of geometrical objects ("generalized orbits", cf. [22], Chapter II, p. 512), and with any element $\mathcal{O}$ of $\mathfrak{S}$ a unitary equivalence class $F(O)$ of factor representations (cf. [22], Theorem 2, p. 551). If $G$ is of type I, all this essentially reduces to the description of $\hat{G}$ given by Auslander and Kostant. For the general case we conjectured previously (cf. [22], p. 463 and [24], p. 78) that, up to quasiequivalence, the set $\{F(O) ; O \in \mathfrak{S}\}$ would yield precisely the collection of all normal representations of $G$. This hypothesis is verified in Theorem 3 of this paper (cf. section 6). In more detail, given $O \in \Im$, let us write $l(O)$ for the quasi-equivalence class of $F(O)$. The the map $l: \subseteq \rightarrow \hat{G}$ is a bijection between $\subseteq$ and $\hat{G}_{\text {norm }}$. In other words, here we have also a canonical bijection between the set of generalized orbits and of characters resp. By virtue of our Theorem 1 this implies a bijection between generalized orbits and Prim $(G)$. This result, suggested first by C. C. Moore, we already established in an earlier paper (cf. [24], Theorem 1, p. 114). Summing up, in the solvable case one has a geometrical construction, involving the coadjoint representation, for all characters.

Assume again, that $G$ is an arbitrary connected and simply connected Lie group. In the course of the proof of Theorem 1 , we associate with any primitive ideal $J$ a locally compact space $\mathcal{A}(J)$, on which $G$ acts as an abelian group (cf. Proposition 1, section 1). Various geometrical properties of $\mathcal{A}(J)$ reflect group theoretic properties of the normal representations belonging to $J$ (cf. Proposition 3, section 3 and Proposition 5, section 4). If $G$ is solvable, there is a $G$ equivariant projection from the generalized orbit belonging to $J$ onto $\mathcal{A}(J)$, and the former is homeomorphic to a product of $A(J)$ with a Euclidean space. By Theorem 1 and by what we said earlier, $J$ determines an essentially unique character $\chi_{J}$. To form this we have to consider traces on factors which, in general, are not of type I. The purpose of Theorem 2 (section 5) is to provide for $\chi_{J}$ a formula, which involves integration over $\mathcal{A}(J)$ of expressions containing only type I traces. This result can be used to extend the formula for the characters of type I solvable groups, established by M. Duflo (cf. [7]) to more general cases.

It appears, that Theorem 1 carries over to an arbitrary separable, locally compact and connected group. Since, however, this extension would require anyhow the results of 
this paper, without adding much to their substance, we decided to postpone it to a later publication.

In our proofs we shall make essential use of parts of our earlier papers (cf. [22], [23] and [24]); the corresponding results will be explicitely stated at the required place.-The reader is assumed to be familiar with the basic results of G. W. Mackey's theory of induced representations (cf. [16]), which we shall often use without special reference.

The reader is urged to consult carefully the Appendix at the end of this paper, which specifies some general assumptions and notational conventions observed throughout. We shall refer to it by $A+$ number of the relevant section.

The results of this paper were announced in [25].

Let $G$ be a connected Lie group with the Lie algebra $\mathfrak{g}$. We shall assume also, that $G$ is simply connected, with the exception of the proof of Theorem $1 . \mathfrak{g}$ can be arbitrary, except in section 6 , where it will be supposed to be solvable.

\section{\$ 1}

The purpose of this section is the proof of Proposition 1, which in itself would already suffice to establish the canonical bijection, quoted in the Introduction, between the set of primitive ideals and of generalized orbits in the solvable case (cf. [24], Theorem 1, p. 113 and section 6 below). We shall follow roughly the line of reasoning employed in [24] for solvable Lie groups. At most of the essential points, however, the proofs presented here substantially differ from those of loc. cit. (compare, in particular, the proof of Lemma 1.1.5, 1.1.9, 1.1.11 and 1.2.5 below with those of Lemma 4, 7, 8 and 28 resp. in [24]).

1.1. By the theorem of Ado, we can identify $\mathfrak{g}$ to a subalgebra of the Lie algebra of all endomorphisms of a finite dimensional real vector space. We denote by $\tilde{\mathfrak{g}}$ the smallest algebraic Lie algebra containing $\mathfrak{g}$. We recall (cf. [2], Théorème $13, \mathrm{p}$. 173), that $[\tilde{\mathfrak{g}}, \tilde{\mathfrak{g}}]=$ $[\mathfrak{g}, \mathfrak{g}]$; we shall write $\mathfrak{D}$ for both sides. Let $\tilde{G}=\exp (\tilde{\mathfrak{g}})$ be a connected and simply connected Lie group belonging to $\tilde{\mathfrak{g}}$ (cf. A.1). Then $G=\exp (\mathfrak{g})$ is a closed, invariant and simply connected subgroup of $\tilde{G}$. We have $[\tilde{G}, \tilde{G}]=[G, G]=\exp (\delta)$, and $L=\exp (\mathrm{D})$ is also a closed, invariant subgroup in $\tilde{G}$.

Lемм 1.1.1. Let $G$ be a connected and simply connected Lie group. Its first derived group is a closed, invariant and type 1 subgroup.

Proof. We recall, that along with $\tilde{\mathfrak{g}}, \mathfrak{b}=[\tilde{\mathfrak{g}}, \tilde{\mathfrak{g}}]$, too, is an algebraic Lie algebra (cf. [2], Théorème 15 , p. 177). Hence the desired conclusion follows from the following result due 
to J. Dixmier (cf. [6], 2.1. Proposition, p. 425): Any connected Lie group, which is locally isomorphic to a real algebraic group, is of type $\mathrm{I}$.

Q.e.d.

The following lemma is routine, but because of the many applications we shall make of it later on, we include here a proof.-Let $M$ be a separable locally compact group and $H$ a closed subgroup of $M$ such that $[M, M] \subset H$. We denote by $¥$ the annihilator of $[M, M]$ in the group $X(H)$ of characters of $H$, and form the direct product $N=M \times \mathcal{F}$. We fix a right-invariant Haar measure $d x$ on $H$ and set for any $b$ in $M: d\left(b x b^{-1}\right)=\Delta(b) \cdot d x$. -We denote by $\mathfrak{S}$ the group $C^{*}$ algebra of $H$ and consider the group of its * automorphisms Aut ( $\mathfrak{g})$ as topologized with pointwise convergence.

Lемма 1.1.2. With the previous notations, there is a continuous representation of $N$ in Aut $(\mathfrak{H})$, such that the action of $a=(b, \chi) \in N$ on the continuous function $f$ with compact support on $H$ is equal to $x \mapsto \chi(x) \cdot f\left(b^{-1} x b\right) / \Delta(b)$.

Proof. Writing af for the last expression, let us show, that $\|a f\|=\|f\|$ in $\mathfrak{S}$. To this end it suffices to settle separately the cases when resp. (1) $a \in M$, (2) $a \in \mathcal{I}$. Ad (1) If $\pi \in \operatorname{Rep}(H)$ (A.4), we have

$$
\pi(a f)=\int_{H} f\left(a^{-1} x a\right) \pi(x) \cdot(d x / \Delta(a))=\int_{H} f(x) \pi\left(a x a^{-1}\right) \cdot d x=\left(a^{-1} \pi\right)(f)
$$

or $\pi(a f)=\left(a^{-1} \pi\right)(f)$. From here we conclude, that the norm of af in $\mathfrak{g}$ is equal to

$$
\sup _{\pi \in \hat{H}}\|\pi(a f)\|=\sup _{\pi \in \hat{H}}\left\|\left(a^{-1} \pi\right)(f)\right\|=\sup _{\pi \in \hat{H}}\|\pi(f)\|=\|f\|,
$$

implying the desired result. Ad (2) If $\chi \in X(H)$ and $f \in \mathcal{L}(H)$ (A.7 and A.8), we have

$$
\|\chi f\|=\sup _{\pi \in \hat{H}}\|\pi(\chi f)\|=\sup _{\pi \in \hat{H}}\|(\chi \pi)(f)\|=\sup _{\pi \in \hat{H}}\|\pi(f)\|=\|f\| .
$$

In this fashion, for each $a \in N$ there is a unique isometry $v \mapsto a v(v \in \mathfrak{g})$, such that for $f \in \mathcal{L}(H) \subset \mathfrak{S}$ we have, if $a=(b, \chi) \in N:(a f)(x) \equiv f\left(b^{-1} x b\right) \chi(x) / \Delta(b)$. That in this manner we obtain a representation of $N$ in Aut $(\mathfrak{S})$ follows from the fact, that this is evidently true if $H$ is replaced by the dense subalgebra $\mathcal{L}(H)$ (A.7). Finally, to prove the continuity of this representation it is enough to observe, that if $a_{n} \rightarrow a$ in $N$ and $f \in \mathcal{L}(H)$, the sequence $\left\{a_{n} f\right\}$ is carried by a fixed compact subset of $H$ and converges uniformly to $f$ Q.e.d.

Corollary. For $a=(b, \chi) \in N$ and $\pi \in \hat{H}$ (A.5) let us define $(a \pi)(x) \equiv \chi(x) \pi\left(b^{-1} x b\right)$. Then $N$ acts on $\hat{H}$ as a topological transformation group.

Proof. We recall (cf. [4], 3.9.9, p. 81), that if $A$ is a $C^{*}$ algebra, $M$ a topological group and $\omega: N \rightarrow$ Aut $(A)$ a continuous homomorphism then, setting for $\pi \in \hat{A}$ and $a \in M:(a \pi)(v) \equiv$ 
$\pi\left(\omega\left(a^{-1}\right)(v)\right) \quad(v \in A), M$ acts on $\hat{A}$ as a topological transformation group.-We write, if $a=(b, \chi) \in N, a^{\prime}=(b, \bar{\chi})$; the map $a \rightarrow a^{\prime}$ is a continuous automorphism of $N$. In this fashion to arrive at the desired conclusion, it suffices to note that, with the notations of our lemma: $(a \pi)(v) \equiv \pi\left(\left(a^{\prime}\right)^{-1}(v)\right)(a \in N, \pi \in \hat{H}, v \in \mathfrak{W})$.

Q.e.d.

Hence, in particular, if $\widetilde{G}$ and $L$ are as at the start, $\widetilde{G}$ acts as a topological transformation group on $\hat{L}$ via the action, which is contragredient to conjugation.

The following lemma is crucial for the rest of this paper; the groundwork for its complex proof was laid by J. Dixmier in [6].

LEMMA 1.1.3. With the previous notations, the orbit space $\hat{L} / \tilde{G}$ is countably separated.

Proof. This will easily follow from the following result (cf. [23], Theorem, p. 379). Assume, that $\mathfrak{h}$ and $\mathrm{g}$ are algebraic Lie algebras of endomorphisms of a finite dimensional real vector space, and that $\mathfrak{h}$ is an ideal in $\mathfrak{g}$. Let us put $H=\exp (\mathfrak{h}) \subset \exp (\mathfrak{g})=G($ A.1). Then $\hat{H} / G$ is countably separated. - To obtain our lemma, it suffices to replace above $\mathfrak{g}$ and $\mathfrak{h}$ by $\tilde{\mathfrak{g}}$ and $\delta=[\tilde{\mathfrak{g}}, \tilde{\mathfrak{g}}]$ resp. In fact, if $\tilde{\mathfrak{g}}$ is an algebraic Lie algebra of endomorphisms, so is ઈ (cf. [2], Théorème 15, p. 177).

Q.e.d.

LEMMA 1.1.4. For each $\pi \in \hat{L}$, there is a canonical choice of a closed subgroup $K_{\pi}$ of $G$, such that $K_{\pi} \supset L$ and that

(i) There is an irreducible representation of $K_{\pi}$, which on $L$ restricts to $\pi$ and, if $\varrho$ is any such representation, $T(\varrho)=\operatorname{ind}_{K \uparrow G} \varrho$ is a factor representation,

(ii) If $\pi_{j} \in \hat{L}$ and $\varrho_{j} \in \hat{K}_{\pi_{j}}, \varrho_{j} \mid L=\pi_{j}(j=1,2), T\left(\varrho_{1}\right)$ and $T\left(\varrho_{2}\right)$ are quasi-equivalent if and only if we have $K_{\pi_{1}}=K_{\pi_{\varepsilon}}$, and $G \varrho_{1}=G \varrho_{2}$.-If this case $T\left(\varrho_{1}\right)$ and $T\left(\varrho_{2}\right)$ are also unitarily equivalent.

(iii) We have for all $\pi \in \hat{L}, a \in \tilde{G}: K_{a \pi} \equiv K_{\pi}$.

Proof. Let $\pi$ be a fixed element of $\hat{L}$; we denote by $G_{\pi}$ its stabilizer in $G($ A.9).-We write $\mathcal{A}$ for the collection of all those closed, connected subgroups of $G$, which contain $L$, and to which $\pi$ admits a $\left(G_{\pi}\right)_{0}(\mathrm{~A} .10)$ invariant extension. We recall (cf. [22], Lemma 3.4, p. 483), that in $\mathcal{A}$ there is a well-defined maximal element $\Pi$. Before proceeding let us note, that if $a$ is some fixed element of $G$, upon substituting $\pi$ by $a \pi$ (A.6) $\Pi$ does not change. In fact, in this case we have $G_{a \pi}=a G_{\pi} a^{-1}=G_{\pi}$ (since $G_{\pi} \supseteq L$ ), and clearly $\mathcal{A}$ does not change. -If $\varrho_{k} \in \Pi$ is such that $\varrho_{k} \mid L=\pi(k=1,2)$, there is a $\chi \in X(\Pi)$ with $\chi \mid L \equiv 1$, and $\varrho_{2}=\chi \varrho_{1}$ (A.8). This implies at once $G_{Q_{1}}=G_{Q_{2}}$; in other words, there is a unique subgroup $\mathfrak{U}_{\pi}$ of $G$, such that if $\varrho \in \hat{\Pi}$ and $\varrho \mid L=\pi, \mathfrak{U}_{\pi}=G_{\varrho}$. We have clearly $\mathfrak{U}_{a \pi} \equiv \mathfrak{U}_{\pi}(a \in \tilde{G})$. -We denote next by $B$ the collection of all those closed subgroups, containing $L$, of $G$, to which $\pi$ admits 
a $\mathfrak{U}_{\pi}$ invariant extension. We recall (cf. [22], Lemma 3.5, p. 484) that $\mathcal{B}$, too, has a unique maximal element $K_{\pi}$. Since under the substitution $\pi \rightarrow a \pi(a \in \widetilde{G}) \mathcal{B}$ does not change, we have also $K_{a \pi} \equiv K_{\pi}$, and thus $K_{\pi}$ satisfies condition (iii) of our lemma. By [22], Lemma 3.7 , p. 484 , it satisfies also (i), and by loc. cit., Lemma 3.8, p. 485 , it verifies (ii). Q.e.d.

Notation 1.1.1. Since $K_{\pi}$ is determined by $E=\tilde{G}_{\pi}$, we shall write sometimes for it $K(E)$, or just $K$, if $E$ is specified by the context.-Similarly, we shall write occasionally $G(E)$ for $G_{\pi}$ - - Observe, that $K(E) \subseteq G(E)$.

Remark. For the benefit of the reader, familiar with our previous paper [22] we mention, that if $G$ is solvable with the Lie algebra $\mathfrak{g}$, we have $K(E)=L \bar{G}_{g}$, where $g$ is an element of $g^{\prime}$ subject to the condition, that the point $\pi \in \hat{L}$, corresponding to the Kirillov orbit $L(g \mid \delta)$, lie in $E$; here $\bar{G}_{g}$ is the reduced stabilizer of $g$. Cf. for all this loc. cit. Proposition 6.1, p. 503 .

LeMma 1.1.5. Let $E$ be a fixed orbit of $\tilde{G}$ in $\hat{L}$. Putting $K=K(E)$, let us set $F=$ $\{\varrho ; \varrho \in \hat{K}, \varrho \mid L \in E\}$. Then $F$, as a subspace of $\hat{K}$, is locally compact and Hausdorff.

Proof. (a) We show first, that $E$ is locally closed in $\hat{L}$. This follows from the following series of observations. (1) By Lemma 1.1.1 $L$ is of type $I$ and thus it is also postliminaire (cf. [4], 9.1. Théorème, (i) $\Rightarrow$ (iii), p. 168), (2) By virtue of (1) $\hat{L}$ is locally quasi-compact and almost Hausdorff (cf. [4], 4.5.7, p. 94 and [10], p. 125), (3) By Corollary to Lemma 1.1.2, $\widetilde{G}$ acts as a topological transformation group on $\hat{L}$, such that $\hat{L} / \widetilde{G}$ is countably separated (ef. Lemma 1.1.3).- - From all this, by virtue of a known theorem of Glimm (cf. [10], Theorem $1,(3) \Rightarrow(2)$, p. 124) we infer, that the $\widetilde{G}$ orbit $E$ in $\hat{L}$ is open in its closure, which is the desired conclusion.

(b) We observe next, that $F$ is locally closed in $R$. To show this, we shall use the following result of Dixmier (cf. [6], 4.2. Lemme, (iii), p. 429). Let us suppose, that $H$ is a closed, invariant and type $I$ subgroup of the separable locally compact group $G$. We assume, that the subset $A \subset \hat{H}$ is locally closed and $G$ invariant. Then, the set $\hat{G}_{A}$ of all those elements in $\hat{G}$ which, when restricted to $H$, are carried by $A$, is locally closed in $\hat{G}$. Substituting, as we can by (a), in place of $G, H$ and $A$ resp. $K, L$ and $E$ we conclude, that $\hat{K}_{E}$ is locally closed in $\hat{K}$. But one shows readily, that $F=\hat{R}_{E}$. In fact, evidently $F \subset \hat{K}_{E}$. If on the other hand, $\varrho$ is some element of $\hat{K}_{E}, \varrho \mid L$ is carried by $E$. Since $K \subset G(E)$ (cf. Notation 1.1.1) $K$ leaves $E$ pointwise fixed, and thus there is $a \pi \in E$ such that $\varrho \mid L \approx \pi$ (A.5). We know, that $\pi$ extends trivially to $K$ (cf. Lemma 1.1.4, (i), which implies $\varrho \mid L=\pi$, or that $\varrho$ belongs to $F$.

(c) Next we recall the following facts; for details of. [4], pp. 61-62. Given a separable $C^{*}$ algebra $A$, we denote by $E(A)$ the family of all nonempty closed subsets of $A$. If $F \in E(A)$, 
we put

$$
\operatorname{ker}(F)=\bigcap_{\pi \in F} \operatorname{ker}(\pi) \subset A
$$

Then (1) The map $F \mapsto \operatorname{ker}(F)$ is a bijection between $E(A)$ and the set of all closed two-sided ideals, different from $A$, (2) If $I=\operatorname{ker}(F)(F \in E(A))$, there is a canonical identification between $\hat{I}$ and $\hat{A}-F$, (3) If $I_{1}, I_{2}$ are closed two-sided ideals of $A$, such that $I_{1} \supset I_{2}$, the dual of the quotient $I_{1} / I_{2}$ is canonically identifiable to $\hat{I}_{1}-\hat{I}_{2}$ - - Let us put $\hat{\Re}=C^{*}(K)$. Since, by (b), $F$ is locally closed in $\hat{K}$, there are open subsets $\hat{K} \supset O_{1} \supset O_{2}$, such that $F=O_{1}-O_{2}$. Thus, by what we have just said, we can find closed two-sided ideals $\Re \supset I_{1} \supset I_{2}$ such that, putting $B=I_{1} / I_{2}$, we have $\hat{B}=F$.

(d) Let us show now, that $B$ is postliminaire (cf. [4], 4.3.1 Definition, p. 87). To this end it is enough to establish, that if $\sigma \in \hat{B}$ (A.5), $\sigma(B)$ contains a nonzero compact operator (cf. [4], 4.3.7, Théorème, p. 89 and 4.1.10 Corollaire, p. 85). Denoting also by $\sigma$ the representation obtained from $\sigma \in \hat{B}$ by lifting it from $B=I_{1} / I_{2}$ to $I_{1}$, there is a $\varrho \in F$, such that $\varrho \mid I_{1}=\sigma$. In this fashion it suffices to prove, that we can find a $v \in \Re$, such that $\varrho(v)$ is nonzero and compact. We denote by $d y$ an element of the Haar measure on $L$, and set $\mathfrak{L}=$ $C^{*}(L)$. One verifies easily, that $\mathfrak{K}$ carries the structure of a left $\mathfrak{Q}$ module, uniquely determined by the condition, that for any $f \in \mathcal{L}(L)(\mathrm{A} .7)$ and $g \in \mathfrak{\Omega}$ we have $f g=\int_{L} f(y) L(y) g \cdot d y$, where $y \mapsto L(y)$ is the left regular representation of $K$ on the underlying space of $\Re$. Furthermore, if $\pi$ is some element of $\operatorname{Rep}(K)$ (A.4) we have $\pi(f g)=\pi(f) \cdot \pi(g)(f \in \mathfrak{L}, g \in \mathfrak{R})$. Let us put $\varrho \mid L=\pi \in \hat{L}$. Since, by Lemma 1.1.1, $L$ is of type $I$ we conclude (cf. [4], 9.1. Théorème, (i) $\Rightarrow$ (iv), p. 168), that there is an $f \in \mathfrak{Q}$ such that $\pi(f)$ is nonzero and compact. If $g \in \mathfrak{\Omega}$ is such, that $\pi(f) \cdot \varrho(g) \neq 0$, then for $v=f g \in \mathscr{R}, \varrho(v)$, too, is nonzero and compact.-We conclude from all this, that $F=\hat{B}$, as a subspace of $\hat{K}$ is locally quasi-compact and almost Hausdorff (cf. [4], 4.5.7, p. 94 bottom, and [10], p. 125).

(e) Let $\mathcal{I}$ be the annihilator of $L$ in $X(K)$. Since $[\tilde{G}, \tilde{G}]=L$, as in Corollary to Lemma 1.1.2, the direct product $N=\tilde{G} \times \mathcal{J}$ acts on $R$ as a topological transformation group. Moreover, if $\varrho$ is any element in $F$, we have $F=N_{\varrho}$. Therefore, since $F$ is locally quasi-compact and almost Hausdorff we conclude (cf. [10], Theorem 1, $(3) \Rightarrow(6)$, p. 124; replace $G$ and $M$ loc. cit. by $N$ and $F$ resp.) that the map $i: N / N_{\varrho} \rightarrow F$, defined by $i\left(a N_{\varrho}\right)=a \varrho(a \in N)$ is a homeomorphism. Hence $N_{\varrho}$ is closed and $F$ is Hausdorff.

Q.e.d.

Notation 1.1.2. Sometimes it will be necessary to indicate the dependence of $F$ on $E \in \hat{L} / \tilde{G}$; if so, we shall write for it $F(E)$.

We set $\mathfrak{A}=\bigcup_{E \in \hat{L} / \tilde{G}} F(E)$ (cf. [22], p. 489; $\mathfrak{A}$ is denoted loc. cit. by (S)).

LEMM A 1.1.6. (i) There is an equivalence relation $\Sigma$ on $\mathfrak{A}$, such that $\varrho_{1}$ is equivalent to 
$\varrho_{2}$ if and only if there is an $E \in \hat{L} / \widetilde{G}$ with $\varrho_{1}, \varrho_{2} \in F(E)$, and then $\varrho_{2} \in \overline{G \varrho_{1}}$, the closure being taken in $F(E)$, (ii) $F(E) / \Sigma$ is countably separated for any $E \in \hat{L} / \tilde{G}$.

Proof. To establish (i), it is enough to show that if, with the notations of the previous lemma, $\varrho_{1}, \varrho_{2} \in F$ and $\varrho_{2} \in \overline{G \varrho_{1}}$, then $\overline{G \varrho_{1}}=\overline{G \varrho_{2}}$. Let again $\varrho$ be fix in $F$. We have $[N, N]=$ $[\tilde{G}, \tilde{G}]=L \subset K \subset N_{\varrho}$, and thus $N_{\varrho}$ is invariant in $N$, and $N / N_{\varrho}$ is abelian. We denote by $A$ the closure of the image of $G$ in $N / N_{e}$ and observe, that the desired conclusion follows from $i(A m)=\overline{G i(m)}\left(m \in N / N_{\varrho}\right)$. As far as (ii) is concerned, evidently $F(E) / \Sigma$ is Hausdorff, hence $F(E) / \Sigma$ is countably separated.

Q.e.d.

Lemma 1.1.5 and 1.1.6 will reappear later in a modified form (cf. Lemma 4.1.4 and 4.1.5 resp.).-We shall see in section 6 , that if $G$ is solvable, there is a natural bijection between $A / \Sigma$ and the collection of all generalized orbits (cf. [22], Chapter II).

Let $L$ be a separable, locally compact and type $I$ group, $T \in \operatorname{Rep}(L)$ and $E$ a subset of $\hat{L}$. As above we shall say, that $T$ is carried by $E$, if the complement of $E$ is of measure zero with respect to the canonical measure corresponding to $T$ on $\hat{L}$.

Lемма 1.1.7. With notations as above, suppose that

$$
T=\int_{\Lambda} T(\lambda) \cdot d \mu(\lambda)
$$

is a direct integral representation of $T$ over the standard measure space $(\Lambda, \mu) . T$ is carried by $E \subset \hat{L}$ if and only if the same is true for $T(\lambda)$ almost everywhere with respect to $\mu$.

Proof. Cf. [24], Lemma 1, p. 87.

Let $A$ be a $C^{*}$ algebra. If $T \in \operatorname{Rep}(A)$ we shall say, that $T$ is homogeneous, if any subrepresentation of $T$ has a kernel equal to $\operatorname{ker}(T)$ (cf. [8], p. 85). Every factor representation is homogeneous. The kernel of any homogeneous representation is primitive (that is, equal to the kernal of an irreducible representation).-Given a group $H$, we shall denote by $\operatorname{hom}(H)$ the set of all homogeneous representations of $C^{*}(H)$.-We recall (cf. Introduction), that Prim $(H)$ stands for the collection of all primitive ideals of $C^{*}(H)$ ([4], 2.9.7, p. 49).

Because of later applications (cf. e.g. Lemma 1.2.5) the following lemma is more general, than immediately needed. $-G$ will denote again a connected and simply connected Lie group.

LemMA 1.1.8. Let $G_{1}$ be a closed subgroup, containing $L=[G, G]$, of $G$. For any $J \in \operatorname{Prim}\left(G_{1}\right)$ there is an orbit $E(J)$ of $\tilde{G}$ on $\hat{L}$, uniquely determined by the property, that if $T \in$ hom $\left(G_{1}\right)$ and ker $(T)=J, T \mid L$ is carried by $E$. 
Proof. The uniqueness is clear.

(a) We show first the existence of an $E(J)$ as above for $T \in \mathrm{Fac}\left(G_{1}\right)\left(\subset \operatorname{hom}\left(G_{1}\right)\right)$ (A.4) and $\operatorname{ker}(T)=J$.-Let us observe first, that if $T_{j} \in \operatorname{Rep}\left(G_{1}\right)$ are such, that $\operatorname{ker}\left(T_{1}\right)=$ $\operatorname{ker}\left(T_{2}\right)$, then we have also $\operatorname{ker}\left(T_{1} \mid L\right)=\operatorname{ker}\left(T_{2} \mid L\right)$. This is well known, but can also be easily established by a remark of (d) of the proof of Lemma 1.1.5. In fact, as loc. cit. we can consider $\mathfrak{G}_{1}=C^{*}\left(G_{1}\right)$ as a left $\mathfrak{L}=C^{*}(L)$ module, such that for any $\pi \in \operatorname{Rep}\left(G_{1}\right)$ we have $\pi(f g)=\pi(f) \pi(g)\left(f \in \mathbb{R}, g \in \mathscr{S}_{1}\right)$. Therefore, if $f \in \operatorname{ker}\left(T_{1} \mid L\right)$, we have also $f \mathscr{S}_{1} \subset \operatorname{ker}\left(T_{1}\right)=$ ker $\left(T_{2}\right)$, and thus $T_{2}(f) T_{2}(g) \equiv T_{2}(f g) \equiv 0\left(g \in \mathscr{S}_{1}\right)$ implying $T_{2}(f)=0$ or $f \in \operatorname{ker}\left(T_{2} \mid L\right)$, and conversely.-Let us observe next, that if $T \in$ Fac $\left(G_{1}\right), T \mid L$ is carried by an element of $\hat{L} / \tilde{G}$. In fact, the canonical measure of $T \mid L$ is $G_{1}$ ergodic on $\hat{L}$ and, by Lemma 1.1.3, $\hat{L} / \tilde{G}$ is countably separated.-We recall ([4], 8.6.8. Proposition, p. 157), that if $A$ is a separable postliminaire $C^{*}$ algebra, $T \in \operatorname{Rep}(A), \mu$ the canonical measure of $T$ on $\hat{A}$ and $h(\mu)$ the closed hull of $\mu$, we have $\operatorname{ker}(T)=\operatorname{ker}(h(\mu))$ (cf. (c), proof of Lemma 1.1.5). Let now $T_{f} \in$ Fac $\left(G_{1}\right)$ be such, that $\operatorname{ker}\left(T_{1}\right)=\operatorname{ker}\left(T_{2}\right)=J$. Since $L$ is of type I (cf. Lemma 1.1.1) we can form the canonical measure $\mu_{j}$ of $T_{1} \mid L$. By what we said above we have $\operatorname{ker}\left(h\left(\mu_{1}\right)\right)=\operatorname{ker}\left(T_{1} \mid L\right)=$ $\operatorname{ker}\left(T_{2} \mid L\right)=\operatorname{ker}\left(h\left(\mu_{2}\right)\right)$ and hence $h\left(\mu_{1}\right)=h\left(\mu_{2}\right)$. Assume, that $T_{j} / L$ is carried by $E_{j} \in \hat{L} / \tilde{G}$. Since $\hat{L} / \tilde{G}$ is countably separated, it is also $T_{0}$ topological space (cf. [10], Theorem 1 , $(3) \Rightarrow(2), p$. 124). Therefore, if $E_{1} \neq E_{2}$, there is an open subset $0 \subset \hat{L}$ such that (say) $E_{1} \subset 0$, and $E_{2} \subset \hat{L}-0$. But then clearly $h\left(\mu_{2}\right) \cap 0$ is empty while $h\left(\mu_{1}\right) \cap 0$ is nonempty, and this contradiction proves, that $E_{1}=E_{2}$.-Summing up, given $J \in \operatorname{Prim}\left(G_{1}\right)$, there is a uniquely determined $E(J) \in \hat{L} / \tilde{G}$, such that if $T \in \operatorname{Fac}\left(G_{1}\right)$ and $\operatorname{ker}(T)=J, T \mid L$ is carried by $E(J)$.

(b) Let now $T \in$ hom $\left(G_{1}\right)$ be such, that $\operatorname{ker}(T)=J$; to prove our lemma, it is enough to show, that $T \mid L$ is carried by $E(J)$ (as above). Let $T=\int_{\Lambda}^{\oplus} T(\lambda) \cdot d \mu(\lambda)$ be a direct integral decomposition of $T$ into factor representations. Since $\operatorname{ker}(T(\lambda)) \equiv J$ almost everywhere with respect to $\mu$ (cf. [8], Lemma 1.9 , p. 91), by (a) $T(\lambda) \mid L$ is carried by $E(J)$ almost everywhere. Since we have also $T \mid L=\int_{\Lambda}^{\oplus}(T(\lambda) \mid L) \cdot d \mu(\lambda)$, by Lemma 1.1 .7 we conclude, that $T \mid L$ is carried by $E(J)$.

Q.e.d.

Notation 1.1.3. If $E=E(J) \in \hat{L} / \widetilde{G}$, we shall write sometimes $K(J), G(J)$ and $F(J)$ for $K(E), G(E)$ and $F(E)$ resp. (cf. Notation 1-2).

Given $\varrho \in \mathfrak{U}$ such that $\varrho \mid L=\pi \in \hat{L}$, as in Lemma 1.1.4 we shall write $T(\varrho)=\operatorname{ind}_{K_{n} \uparrow G} \varrho$ $\in \operatorname{Fac}(G)$. Putting $J(\varrho)=\operatorname{ker}(T(\varrho))$, we have $J(\varrho) \in \operatorname{Prim}(G)$ (cf. [3], Corollaire 3, p. 100).The main objective of section 1 is to establish, that the map $\varrho \mapsto J(\varrho)(\varrho \in \mathscr{U})$ gives rise to a bijection between $\mathfrak{A} / \mathbf{\Sigma}$ and $\operatorname{Prim}(G)$. 
LEMMA 1.1.9. With the previous notations, assume that $\varrho_{j} \in \mathfrak{Y}$ are such, that $J\left(\varrho_{1}\right)=$ $J\left(\varrho_{2}\right)\left(=J\right.$, say). Then $\varrho_{1}$ and $\varrho_{2}$ lie on the same $\Sigma$ orbit $\mathfrak{A}$ (cf. Lemma 1.1.6).

Proof. Let us show first, that if $\varrho \in \mathfrak{A}$ satisfies $J(\varrho)=J$, we have $\varrho \in F(J)$. In fact, by Lemma 1.1.8 $T(\varrho) \mid L$ is carried by $E(J)$. On the other hand, if $\varrho \mid L=\pi \in \hat{L}$, we have (A.11) $T(\varrho) \mid L=\int_{G / K_{\pi}}^{\oplus} \sigma(\varrho \mid L) \cdot d \sigma$. Since the right-hand-side is carried by $G \pi$, we have $\varrho \mid L=\pi \in E(J)$ and thus $\varrho \in F(J)$. We write $K=K(J)$ and $F=F(J)$. In this fashion, by $J\left(\varrho_{1}\right)=J\left(\varrho_{2}\right)=J$ we obtain $\varrho_{j} \in F(j=1,2)$. To complete the proof of our lemma, we must show, that the closures of $G \varrho_{j}$ in $F$ coincide. By (c) of the proof of Lemma 1.1.5 there are closed two-sided ideals $I_{j}$ in $C^{*}(K)$ such that $I_{1} \supset I_{2}$ and, putting $B=I_{1} / I_{2}$, we have $F=\hat{B}$. This means, that $F$ is the set $\left\{\varrho ; \varrho \in \hat{K}, \operatorname{ker}(\varrho) \supset I_{2}, \operatorname{ker}(\varrho) \ngtr I_{1}\right\}$. Noting that $\operatorname{ker}\left(T\left(\varrho_{j}\right) \mid K\right) \supset I_{2}$, let us write $T_{j}^{\prime}$ for the representations, corresponding to $T\left(\varrho_{j}\right)$, of $B$; these are clearly nondegenerate. Furthermore, by ker $\left(T\left(\varrho_{1}\right)\right)=J\left(\varrho_{1}\right)=J\left(\varrho_{2}\right)=\operatorname{ker}\left(T\left(\varrho_{2}\right)\right)$, we have ker $\left(T_{1}^{\prime}\right)=$ $\operatorname{ker}\left(T_{2}^{\prime}\right)$. We recall (cf. (d), proof of Lemma 1.1.5), that $B$ is postliminaire. The closed hull of the canonical measure corresponding to $T_{j}^{\prime}$ on $B$ is equal to the closure $\overline{G \varrho_{j}}$ of $G \varrho_{j}$ in $F$. Reasoning as in the previous lemma we conclude therefore, that $\operatorname{ker}\left(T_{1}^{\prime}\right)=\operatorname{ker}\left(T_{2}^{\prime}\right)$ implies $\overline{G \varrho_{1}}=\overline{G \varrho_{2}}$, completing the proof of our lemma.

Q.e.d.

LEMMA 1.1.10. Suppose, that $G$ is a separable locally compact group. (i) If $K$ is a closed subgroup, and $T_{j} \in \operatorname{Rep}(K)$ are such, that $\operatorname{ker}\left(T_{1}\right) \subseteq \operatorname{ker}\left(T_{2}\right)$ then, writing $V_{j}=\operatorname{ind}_{K \uparrow G} T_{j}$ we have $\operatorname{ker}\left(V_{1}\right) \subseteq \operatorname{ker}\left(V_{2}\right)$. (ii) If $K=G, S \in \operatorname{Rep}(G)$ and $W_{j}=S \otimes T$, we get $\operatorname{ker}\left(W_{1}\right) \subseteq$ $\operatorname{ker}\left(W_{2}\right)$.

Proof. For this of. [24], Corollaries 1-2 to Lemma 15, p. 99.

Lғмм 1.1.11. Suppose, that the elements $\varrho_{1}, \varrho_{2}$ of $\mathfrak{U}$ lie on the same $\Sigma$ orbit. Then we have $J\left(\varrho_{1}\right)=J\left(\varrho_{2}\right)$.

Proof. By the proof of Lemma 1.1 .9 our assumption implies, that $\operatorname{ker}\left(T\left(\varrho_{1}\right) \mid K\right)=$ $\operatorname{ker}\left(T\left(\varrho_{2}\right) \mid K\right) \quad\left(K=K(E)\right.$ if $\left.\varrho_{1}, \varrho_{2} \in F(E)\right)$. On the other hand, the representation ind $_{K \uparrow G}\left(T\left(\varrho_{k}\right) \mid K\right)$ is quasi-equivalent to $T\left(\varrho_{k}\right)$ and hence, in particular, their kernels coincide $(k=1,2)$. Therefore by (i), Lemma 1.1 .10 we conclude, that $J\left(\varrho_{1}\right)=\operatorname{ker}\left(T\left(\varrho_{1}\right)\right)=$ $\operatorname{ker}\left(T\left(\varrho_{2}\right)\right)=J\left(\varrho_{2}\right)$. Q.e.d.

1.2. By what we have just seen, the map $\varrho \mapsto J(\varrho)(\varrho \in \mathfrak{A})$ gives rise to an injection of $\mathfrak{A} / \Sigma$ into Prim $(G)$. Our next objective will be to show, that the said map is actually a bijection. To this end we have to show, that any primitive ideal of $C^{*}(G)$ is of the form $J(\varrho)(\varrho \in \mathfrak{Y})$. 
As we shall see below (cf. Lemma 1.2.3) this is equivalent to the following: For each $J \in \operatorname{Prim}(G)$ there is a factor representation $V$ with $\operatorname{ker}(V)=J$, and such that $V \mid L$ gives rise to a transitive quasi-orbit on $\hat{L}$.

Lемм а 1.2.1. Given $J \in \operatorname{Prim}(G)$, there is a $\varrho F(J)$ such that $J(\varrho) \subseteq J$.

Proof. We write $K=K(J)$ (cf. Notation 1.1.3) and recall, that $F(J) \subset \hat{K}$ (cf. Lemma 1.1.5).

(a) Suppose, that $T \in \mathrm{Fac}(G)$, is such, that $\operatorname{ker}(T)=J$. We set $T^{\prime}=\operatorname{ind}_{K \uparrow G}(T \mid K)$ and show, that $T^{\prime} \in \mathrm{Fac}(G)$ and that $\operatorname{ker}\left(T^{\prime}\right)=J(\varrho)$, where $\varrho$ is some element in $F(J)$. -We start by recalling (cf. [4], 8.4.2, Théorème, p. 149) that there is a standard measure $\mu$ on the quasi-dual $\hat{K}$ of $K$, and a $\mu$ measurable cross-section $\zeta \mapsto T(\zeta)$ from $\hat{K}$ into $\operatorname{Fac}(K)$ (A.4), such that $T \mid K=\int_{\hat{K}} T(\zeta) \cdot d \mu(\zeta)$. Putting $A=\{\zeta ; \zeta \in \hat{K}, T(\zeta) \mid L$ is carried by $E(J)\}$ we show next, that the complement of $A$ in $\hat{K}$ is of $\mu$ measure zero. In fact, since $\operatorname{ker}(T)=J$, by Lemma 1.1.8, $T \mid L$ is carried by $E(J)$. On the other hand we have $T \mid L=\int_{\hat{K}}^{\oplus}(T(\zeta) \mid L) \cdot d \mu(\zeta)$ and hence, by Lemma 1.1.7, $T(\zeta) \mid L$ is carried by $E(J)$ almost everywhere with respect to $\mu$, which is the desired conclusion.-Let $F^{\prime}$ be the image of $F$ in $\hat{K}$ under the canonical injection of $\hat{K}$ into $\hat{K}$. We observe, that $A \subset F^{\prime}$. In fact, if $\zeta \in A$, by definition, $T(\zeta) \mid L$ is carried by $E(J)$. Since $K \subset G(J), T(\varrho) \mid L \approx \pi($ A.5) $(\pi \in E(J))$ and thus since $T(\zeta) \in \mathrm{Fac}(K), T(\zeta) \approx \varrho(\varrho \in F(J))$.-We recall (cf. [4], 7.3.6, Théorème, p. 139) that the image $\hat{K}_{I} \subset \hat{K}$ of $\hat{K}$ under the canonical injection is a Borel set, and that the said map is a Borel isomorphism. $F(J)$ is locally closed in $\widehat{K}$ (cf. (b) proof of Lemma 1.1.5) and hence a Borel subset in $\hat{K}$. Using (d) loc. cit and [4], 4.6.2. Proposition, p. 95 (or by direct verification using (e) loc. cit.) we conclude, that there is a Borel cross section $\zeta \mapsto \varrho(\zeta)$ from $F(J)$ into $\operatorname{Irr}(K)$. From all this we infer, that there exist a Radon measure $v$ on $F(J)$, such that $T \mid K=\int_{F^{(}(J)}^{\oplus} \varrho^{\prime}(\zeta) \cdot d v(\zeta)$ where $\varrho^{\prime}(\zeta)$ is of the form $N \cdot \varrho(\zeta)$, and the positive integer $N(\leqslant+\infty)$ does not depend on $\zeta$. The measure $v$ is quasi-invariant with respect to $G$ and, since $T \in \mathrm{Fac}(G)$, it is also ergodic. The equivalence relation $\Sigma$ on $F(J)$ (cf. Lemma 1.1.6) is $G$ invariant, such that $F(J) / \Sigma$ is countably separated (cf. (ii) loc. cit.). Therefore $v$ is carried by a $\Sigma$ orbit $\mathcal{A} \subset F(J)$, and thus we can write $T \mid K=\int_{A}^{\otimes} \varrho^{\prime}(\zeta) \cdot d \nu(\zeta)$. Putting $V(\zeta)=\operatorname{ind}_{K \uparrow G} T(\zeta)$ we get (cf. [16], Theorem 10.1, p. 123) $T^{\prime}=\operatorname{ind}_{K \uparrow G}(T \mid K)=\int_{A}^{\oplus} V(\zeta) \cdot d v(\zeta)$. Since $V(\zeta)$ is unitarily equivalent to $V\left(\zeta^{\prime}\right)$ if $G \zeta=G \zeta^{\prime}$, a routine reasoning (cf. e.g. [22], Lemma 2.3.1, p. 549) shows, that by virtue of the $G$ ergodicity of $v, T^{\prime} \in$ Fac $(G)$. This implies, that $\operatorname{ker}\left(T^{\prime}\right)=\operatorname{ker}(V(\zeta))$ almost everywhere with respect to $v$ (cf. [8], Lemma 1.9, p. 91). But ker $(V(\zeta))$, being of the form $J(\varrho)(\varrho \in F(J))$, the same is valid for ker $\left(T^{\prime}\right)$.

(b) Summing up, we have shown above, that $T^{\prime}=\operatorname{ind}_{K \uparrow G}(T \mid K)$ is a factor representa- 
tion of $G$, such that $\operatorname{ker}\left(T^{\prime}\right)=J(\varrho)$ for some $\varrho$ in $F(J)$. To complete the proof of our lemma, it will therefore suffice to establish, that $\operatorname{ker}(T) \supseteq \operatorname{ker}\left(T^{\prime}\right)$. This, however, can be verified by a trivial modification of the proof of Lemma 10 in [24] (p. 95) to which the reader is referred for further details.

Q.e.d.

Lемм 1.2.2. Suppose, that $\Gamma$ is a central extension by the circle group of a direct product of a vector group with a free abelian group. Let $\mathbf{T}$ be the circle group in the center of $\Gamma$ and assume, that the factor representation $V$ of $\Gamma$, when restricted to $\mathbf{T}$, is a multiple of the identity map of $\mathbf{T}$ onto itself. Then there is a character $\chi$ of the center $U^{\#}$ of the centralizer of the connected center of $\Gamma$, such that $\operatorname{ker}(V)=\operatorname{ker}\left(\operatorname{ind}_{U^{*} \uparrow \Gamma} \chi\right)$

Proof. Let us write $J=\operatorname{ker}(V)$ and observe, that if $U \in \operatorname{Rep}(\Gamma)$ is such, that $\operatorname{ker}(U)=J$, we have also $\operatorname{ker}(V \mid \mathbf{T})=\operatorname{ker}(U \mid \mathbf{T})$, and thus $U \mid \mathbf{T}$ is a multiple of the identity map of the circle group onto itself. This being said, the desired conclusion follows from [24], Proposition 3, p. 104.

Q.e.d.

Lemma 1.2.3. For any $J$ in Prim $(G)$ the following two conditions are equivalent (1) There is a $\varrho \in \mathfrak{A}$, such that $J=J(\varrho)$, (2) There is a $V \in F a c(G)$, such that $\operatorname{ker}(V)=J$, and $V \mid L$ gives rise to a transitive quasi-orbit on $\hat{L}$.

Proof. (1) $\Rightarrow(2)$ The assumption means, that $J$ is the kernel of a representation $T(\varrho)=$ ind $_{K \uparrow G} \varrho$, where $\varrho \mid L=\pi \in \hat{L}$, and $K=K_{\pi}$ (cf. Lemma 1.1.4). By (i) loc. cit. we have $T(\varrho) \in$ Fac $(G)$, and $T(\varrho) \mid L$ is carried by $G \pi$. Hence we can take $V=T(\varrho)$.

$(2) \Rightarrow(1)$ Assume now, that $V \in \operatorname{Fac}(G)$ is such, that $\operatorname{ker}(V)=J$ and $V \mid L$ is carried by $G \pi \subset \hat{L}$. Let $\pi^{e}$ be a projective extension of $\pi$ to $G_{\pi}, G_{\pi}^{e}$ the corresponding central extension of $G_{\pi}$ by the circle group and $\phi$ the canonical homomorphism from $G_{\pi}^{e}$ onto $K_{\pi}^{e} / L=M_{\pi}$ (cf. for all this [22], pp. 480-481). Then $M_{\pi}$ satisfies the condition imposed on $\Gamma$ in Lemma 1.2.2. Let $\mathcal{T}(\mathbf{T})$ be the circle group in $\left(M_{\pi}\right)^{*}(\mathrm{~A} .12)$ (in $\left(G_{\pi}^{e}\right)^{*}$ resp.). Given a representation, constant on $\mathbf{T}$, of $G_{\pi}^{e}$, we write $[U]^{\sim}$ for the corresponding representation of $G_{\pi}$. This being so, since $V \mid L$ gives rise to a transitive quasi-orbit on $\hat{L}$, there is a $\tau \in$ Fac $\left(M_{\pi}\right)$, such that $\tau \mid \mathcal{T}$ is the conjugate of the identity map of $\mathcal{T}$ onto itself, and that $V=\operatorname{ind}_{G_{\pi} \uparrow G}\left[\pi^{e} \otimes(\tau \circ \phi)\right]^{\sim}$. If $U^{\#}$ is the center of the centralizer of $\left(M_{\pi}^{\#}\right)_{0}$, by Lemma 1.2.2 there is a $\chi \in X\left(U^{*}\right)$ such that $\operatorname{ker}(\tau)=\operatorname{ker}(V(\chi))$ where $V(\chi)=\operatorname{ind}_{U^{*} \uparrow M_{\pi}} \chi$. Clearly $\chi \mid \mathcal{T}$ is the identity map of $\mathcal{J}$ onto itself. Let us put $V^{\prime}=\operatorname{ind}_{G_{\pi} \uparrow G}\left[\pi^{e} \otimes(V(\chi) \circ \phi)\right]^{\sim}$. By the proof of Lemma 3.7 in [22] (p. 484), there is a $\varrho \in \mathfrak{A} \cap K_{\pi}$ such that $V^{\prime}=$ ind $_{K_{\pi} \uparrow G}$, and hence ker $\left(V^{\prime}\right)=J(\varrho)$. Therefore, in order to complete the proof of our lemma, it is enough to show, that $\operatorname{ker}(V)=$ $\operatorname{ker}\left(V^{\prime}\right)$. To this end we recall the following well-known statement. Suppose, that $G$ is a 
separable, locally compact group, $H$ a closed, invariant subgroup and $\Phi$ the canonical homomorphism from $G$ onto $G / H=A$. Then, if $V_{1} \in \operatorname{Rep}(A), \operatorname{ker}\left(V_{1}\right)=\operatorname{ker}\left(V_{2}\right)$ is equivalent to $\operatorname{ker}\left(V_{1} \circ \Phi\right)=\operatorname{ker}\left(V_{2} \circ \Phi\right)$. By a repeated application of this and of Lemma 1.1.10 one concludes at once, that

$$
\left.\operatorname{ker}(V)=\underset{G_{\pi} \uparrow G}{\operatorname{ker}(\operatorname{ind}}\left[\pi^{e} \otimes(\tau \circ \phi)\right]^{\sim}\right)=\operatorname{ker}\left(\operatorname{ind}_{G_{\pi} \uparrow G}\left[\pi^{e} \otimes(V(\chi) \circ \phi)\right]^{\sim}\right)=\operatorname{ker}\left(V^{\prime}\right)
$$

Q.e.d.

LEMMA 1.2.4. Suppose, that for $J \in \operatorname{Prim}(G)$ there is a factor representation $V$, such that $\operatorname{ker}(V) \supseteq J$ and that $V \mid L$ gives rise to a transitive quasi-orbit carried by $E(J)$ (cf. Lemma 1.1.8). Then $J$ is of the form $J(\varrho)(\varrho \in \mathfrak{Q})$.

Proof. Let us set $\operatorname{ker}(V)=J_{1} \in \operatorname{Prim}(G)$. We claim, that $E(J)=E\left(J_{1}\right)$. In fact, by assumption, the quasi-orbit of $V \mid L$ is carried by $E(J)$. On the other hand, by Lemma 1.1.8, it is also carried by $E\left(J_{1}\right)$, and thus $E(J)=E\left(J_{1}\right)$. Hence also $F(J)=F\left(J_{1}\right)$. Next we observe that $J_{1}$, by definition, satisfies condition (2) of the previous lemma; therefore there is a $\varrho_{1} \in \mathfrak{U}$ such that $J\left(\varrho_{1}\right)=J_{1} \supseteq J$. As in the proof of Lemma 1.1 .9 we conclude, that $\varrho_{1} \in F\left(J_{1}\right)=F(J)$. On the other hand, by Lemma 1.2.1 there is a $\varrho_{2} \in F(J)$ such that $J \supseteq J\left(\varrho_{2}\right)$. Summing up, by what we have just seen, to complete the proof of our lemma it is enough to show, that if $\varrho_{1}, \varrho_{2} \in F(J)$ are such, that $J\left(\varrho_{1}\right) \supseteq J\left(\varrho_{2}\right)$, then $J\left(\varrho_{1}\right)=J\left(\varrho_{2}\right)$. Putting $K=$ $K(J)$, let us write again $T\left(\varrho_{j}\right)=\operatorname{ind}_{K \uparrow G} \varrho_{j}$. Our hypothesis implies, that $\operatorname{ker}\left(T\left(\varrho_{1}\right)\right) \supseteq$ $\operatorname{ker}\left(T\left(\varrho_{2}\right)\right)$ whence also $\operatorname{ker}\left(T\left(\varrho_{1}\right) \mid K\right) \supseteq \operatorname{ker}\left(T\left(\varrho_{2}\right) \mid K\right)$. Reasoning similarly, as in the proof of Lemma 1.1.9, we deduce from this, that $\overline{G \varrho_{1}} \supseteq \overline{G \varrho_{2}}$, closures being formed in $F(J)$. By the proof of Lemma 1.1.6, however, we have then $\overline{G \varrho_{1}}=\overline{G \varrho_{2}}$ which, by Lemma 1.1.11 implies, that $J\left(\varrho_{1}\right)=J\left(\varrho_{2}\right)$, completing the proof of our lemma.

Q.e.d.

Lемма 1.2.5. For any element $J$ of Prim $(G)$, there is a factor representation $V$ of $G$, such that $\operatorname{ker}(V) \supseteq J$, and that $V \mid L$ gives rise to a transitive quasi-orbit, carried by $E(J)$.

Proof. We recall (cf. Notation 1.1.3), that $G(J) \equiv G_{\pi}(\pi \in E(J)$.

(a) Let $T \in \operatorname{Irr}(G)$ be such, that $\operatorname{ker}(T)=J$; then $T \mid L$ is carried by $E(J) \subset \hat{L}$. We denote by $\varrho$ a fixed element of $E(J)$. Using a reasoning already employed (cf. (e), proof of Lemma 1.1.5) we show, that the map $j: \tilde{G} / \tilde{G}_{e} \rightarrow E(J)$ defined by $j\left(a \tilde{G}_{e}\right)=a \varrho(a \in \tilde{G})$ is a homeomorphism. In fact, $\hat{L}$ is locally quasi-compact and almost Hausdorff (cf. (a), proof of Lemma 1.1.5) and $\hat{L} / \tilde{G}$ is countably separated (Lemma 1.1.3) and thus the desired conclusion is implied by a theorem of Glimm (cf. [10], Theorem $1,(3) \Rightarrow(6)$, p. 124). Since $\tilde{G}_{\varrho}$ is closed, and evidently $\tilde{G}_{Q} \supset L, \widetilde{G} / \tilde{G}_{Q}$ is a connected abelian Lie group. Since $T \in \operatorname{Irr}(G)$ and 
$j$ is $G$ equivariant, $T \mid L$ is carried by the relative closure of a $G$ orbit in $E(J)$. Therefore from now on we can assume to be given the following situation. There is a connected abelian Lie group $\mathcal{A}$, a continuous homomorphism $\varphi$ of $G$ into $\mathcal{A}$ such that $\operatorname{ker}(\varphi)=G(J)$, and that $\mathcal{A}^{\prime}=\varphi(G)$ implies $\overline{\mathcal{A}^{\prime}}=\mathcal{A}$; there is furthermore a Borel injection $\pi: \mathcal{A} \rightarrow \operatorname{Irr}(L)$ with (writing $a \varrho$ in place of $\varphi(a) \zeta$ for $a \in G$ and $\zeta \in \hat{L}$ ) $a \pi(\zeta) \equiv \pi(a \zeta)$ (A.5) and a Borel measure $\mu$ on $\mathcal{A}$ such that

$$
T \mid L=\int_{A}^{\oplus} \pi^{\prime}(\xi) \cdot d \mu(\xi)
$$

where $\pi^{\prime}(\zeta) \approx \pi(\zeta)(\zeta \in \mathcal{A})$ (A.5).

(b) Here and in Lemma 4.2.1 we shall use the following elementary result. Suppose, that $\mathcal{A}$ is a connected abelian Lie group and $\mathcal{A}^{\prime}$ a connected dense subgroup. There is a closed, connected subgroup $\mathcal{A}_{1}$ such that $\mathcal{A}_{1} \cdot \mathcal{A}^{\prime}=\mathcal{A}$, and putting $\mathcal{A}_{1}^{\prime}=\mathcal{A}^{\prime} \cap \mathcal{A}_{1}, \mathcal{A}_{1}^{\prime}$ is a countable dense subgroup of $\mathcal{A}_{1}$.

(c) We apply now (b) to $\mathcal{A}$ and $\mathcal{A}^{\prime}$ as at the end of (a). We write $\mathfrak{B}=\mathcal{A} / \mathcal{A}_{1}$ and denote by $\omega$ the canonical homomorphism from $\mathcal{A}$ onto $\mathfrak{B}$. If $\omega^{\prime}=\omega \circ \varphi, \omega^{\prime}$ is a homomorphism of $G$ onto $\mathfrak{B}$. Putting $S=\operatorname{ker}\left(\omega^{\prime}\right), S$ is a closed subgroup of $G$, such that $S / G(J)$ is discrete and $\mathfrak{B}=A / A_{1}$ is isomorphic to $G / S$.-This being so, we are going to show, that there is a $T_{1} \in \operatorname{Irr}(S)$ with $T=\operatorname{ind}_{S \uparrow G} T_{1}$. In fact, let us denote by $\mathcal{D}$ the spectral measure, defined on the Borel sets of $\mathcal{A}$ and taking its values in $[R(T \mid L)]^{*}$ (A.2, A.12), corresponding to the decomposition (+). If $Q$ is its direct image, via $\omega$, on $\mathfrak{B}=G / S,(T, Q)$ constitutes a transitive system of imprimitivity based on $G / S$, and hence there is indeed a $T_{1} \in \operatorname{Rep}(S)$ such that $T=\operatorname{ind}_{S \uparrow G} T_{1}$ (cf. [19], Theorem 6.6, p. 291). Moreover $T_{1} \in \operatorname{Irr}(S)$, since, by assumption, $T \in \operatorname{Irr}(G)$.

(d) Writing $\mathcal{J}=\operatorname{ker}\left(T_{1}\right) \in \operatorname{Prim}(S)$, we form $E(\mathcal{J}) \in \hat{L} / \tilde{G}$ (replace in Lemma $1.1 .8 G_{1}$ through $S)$. We claim, that $E(\mathcal{F})=E(J)$. In fact, by $T=$ ind $_{S \uparrow G} T_{1}$ we have $T \mid L=$ $\int_{G / S}^{\oplus} \tau\left(T_{1} \mid L\right) \cdot d \tau(\mathrm{A} .1 \mathrm{I})$. Since $T \mid L$ is carried by $E(J)$ so is, by Lemma 1.1.7, $T_{1} \mid L$. The latter is carried, however, also by $E(\mathcal{Y})$, since $\mathcal{f}=\operatorname{ker}\left(T_{1}\right)$; hence $E(\mathcal{F})=E(J)$. - Let us put $H=S_{0}(\mathrm{~A} .10)$. Since, if $V_{j} \in \operatorname{Rep}(S)$ and $\operatorname{ker}\left(V_{1}\right)=\operatorname{ker}\left(V_{2}\right)$ we have also $\operatorname{ker}\left(V_{1} \mid H\right)=$ $\operatorname{ker}\left(V_{2} \mid H\right)$, we can speak of the closed two-sided ideal $\mathcal{J}$ of $C^{*}(H)$, which is the restriction of $\mathcal{Y}$ to $H$; we shall denote it also by $\mathcal{Y} \mid H$. Next we show, that there is a $\sigma \in \operatorname{Prim}(H)$, such that $\mathcal{I} \subset \sigma$ and $E(\sigma)=E(J)$ (cf. Lemma 1.1.8). To this end we recall (cf. [8], p. 97, top), that we can find a Borel measure $\nu$ on $\operatorname{Prim}(H)$ and a $\nu$ measurable cross-section $\varrho$ : $\operatorname{Prim}(H) \rightarrow$ hom $(H)$, such that

$$
T_{1} \mid H=\int_{\operatorname{Prim}(H)}^{\oplus} \varrho(\lambda) \cdot d \nu(\lambda) \quad(\operatorname{ker}(\varrho(\lambda)) \equiv \lambda)
$$


Also (cf. loc. cit. Lemma 3.4, p. 99), denoting by $h(v)$ the closed hull of $\nu$ and by ker $(h(v)$ ) the intersection of the corresponding primitive ideals, we have $J=\operatorname{ker}\left(T_{1} \mid H\right)=\operatorname{ker}(h(v))$. Since at the same time

$$
T_{1} \mid L=\int_{\operatorname{Prim}(H)}^{\oplus}(\varrho(\lambda) \mid L) \cdot d \nu(\lambda)
$$

and, by what we saw above, $T_{1} \mid L$ is carried by $E(J)$, the desired conclusion follows from Lemma 1.1.7.

(e) Let $U \in \operatorname{Irr}(H)$ be such, that $\operatorname{ker}(U)=\sigma$. We claim, that $U \mid L$ is a multiple of some element $\pi$ of $E(J)$. In fact, $U \mid L$ is carried by $E(\sigma)=E(J)$. Since $H=(G(J))_{0}$, the action of $H$ on $E(J)$ is trivial; whence the result.

(f) For more details about the following ef. [24], p. 103.-Let $P(H)$ be the set of all continuous positive definite functions on $H$. We denote by $P_{y}$ the subset of $P(H)$ composed of all those elements, which give rise to a unitary representation of $H$ the kernel of which contains $\mathcal{J}$. Let $E P_{\mathfrak{y}}$ be the set of all extremal elements of $P_{y}$. We define analogously $E P_{y} \subset P(S)$. This being said let $\varphi$ be a positive definite function belonging to $U$ and such that $\varphi(e)=1$. Then $\varphi \in E P_{\mathcal{J}}$ and there is an element $\omega \in E P_{\ni}$ such that $\omega \mid H=\varphi$ (cf. [24], Lemma 19, p. 103). Denoting by $Z$ the unitary representation of $S$ determined by $\omega$, there is a countable subset $B \subset S$ such that $Z \mid L=\Sigma_{b \in B}^{\oplus} b U$ (cf. [24], Lemma 20, p. 104).

(g) Setting $V^{\prime}=$ ind $_{S \uparrow G} Z$ we are going to show next, that (1) $\operatorname{ker}\left(V^{\prime}\right) \supseteq J,(2) V^{\prime} \mid L$ is carried by $G \pi \subset \hat{L}$ where $\pi$ is as in (e) above. Ad (1) $\omega \in P_{\ni}$ yields $\operatorname{ker}(Z) \supseteq \mathcal{Y}=\operatorname{ker}\left(T_{1}\right)$. Since, by (c) above, $T=\operatorname{ind}_{S \uparrow G} T_{1}$, and since $\operatorname{ker}(T)=J$, the desired conclusion follows from Lemma 1.1.10, (i). Ad (2) This follows at once from $V^{\prime} \mid L=\int_{G / S} \tau(Z \mid L) \cdot d \tau(\mathrm{A} .11)$ and from $Z \mid L \approx \sum_{b \in B}^{\oplus} b_{\pi}$ (A.5 and (f) above).

(h) Reasoning as in (d) above, by considering a direct integral decomposition of $V^{\prime}$ into homogeneous representations over Prim $(G)$ we conclude, that there is a $U \in$ hom $(G)$, such that $\operatorname{ker}(U) \supseteq J$ and $U \mid L$ is carried by $G \pi$. Let $U=\int_{\Lambda}^{\oplus} T(\lambda) \cdot d \alpha(\lambda)$ be the central decomposition of $U$. We have $\operatorname{ker}(U) \equiv \operatorname{ker}(T(\lambda)$ ) almost everywhere with respect to $\alpha$ (cf. [8], Lemma 1.9 , p. 91). Hence, again by the reasoning of (d), there is a $\lambda_{0} \in \Lambda$ such that if $V=T\left(\lambda_{0}\right)$, we have $V \in \operatorname{Fac}(G)$, $\operatorname{ker}(V) \supseteq J$ and $V \mid L$ is carried by $G \pi \subset E(J)$. In other words, $V$ satisfies the conditions of our lemma.

Q.e.d.

We sum up the results of the previous discussion in the following

Proposition 1. Given $\varrho \in \mathfrak{A}$ such that $\varrho \mid L=\pi \in \hat{L}$ let $u s$ write

$$
J(\varrho)=\operatorname{ker}\left(\operatorname{ind}_{K_{\pi} \uparrow G} \varrho\right) \in \operatorname{Prim}(G)
$$

Then (i) The map $J: A \rightarrow \operatorname{Prim}(G)$ is surjective, (ii) We have $J\left(\varrho_{1}\right)=J\left(\varrho_{2}\right)$ if and only if $\varrho_{1}$ 
and $\varrho_{2}$ lie on the same $\Sigma$ orbit in $\mathfrak{A}$ (cf. Lemma 1.1.6) (iii) For a fixed $J \in$ Prim $(G)$ let us set $\mathcal{A}(J)=\{\varrho ; \varrho \in \mathcal{A}, J(\varrho)=J\}$. Then $\mathcal{A}(J)$, as a subspace of the dual of $K(J)$ (cf. Notation 1.1.3) is locally compact and Hausdorff. Given a factor representation $V$ of $G$ with a kernel equal to $J$, there is an (up to equivalence) unique Radon measure $\mu$ on $F(J)$, quasi-invariant and ergodic with respect to the action of $G$, such that $V \mid K(J) \approx \int_{A(J)}^{\oplus} \varrho \cdot d \mu(\varrho)$.

Proof. Ad (i) Follows from Lemma 1.2.4 and 1.2.5, Ad (ii) Follows from Lemma 1.1.9 and 1.1.11, Ad (iii) Follows from Lemma 1.1.5 and (a) of the proof of Lemma 1.2.1. Q.e.d.

\section{\$2}

The purpose of this Section II is to construct a normal representation (cf. Introduction or II.3 below) the kernel of which coincides with a given primitive ideal $J$ (cf. Proposition 2). We shall accomplish this in the following manner. It results from our previous considerations (cf. (a) of the proof of Lemma 1.2.5 or II.2 below) that $E(J)$ carries a $G$ invariant Radon measure $\omega$. Forming $U=\operatorname{ind}_{L \uparrow G}\left(\int_{E(J)}^{\oplus} \pi \cdot d \omega(\pi)\right)$, we shall show first, that $U$ is a representation with trace, or that there is a faithful, normal, semifinite trace $\phi$ on $[R(U)]^{+}$such that, writing $\mathscr{S H}^{*}=C^{*}(G)$, the family of operators $\left\{A ; A=U(a), a \in \mathcal{S}^{+}\right.$and $\phi(A)<+\infty$ ) generates $R(U)$ (cf. Lemma 2.3.4). Using this the desired normal representa. tion will be obtained through central decomposition of $U$; it will have the form of the direct integral in (iii), Proposition 1 , provided $\mu$ loc. cit. is a $G$ invariant Radon measure on $\mathcal{A}(J)$ (cf. Lemma 2.3.5 and Lemma 2.3.6).- - In order to establish the statement concerning $U$, we shall have to include $\mathcal{L}(G)($ A.7) in a larger * invariant subalgebra $\mathcal{B}$ of $(\mathfrak{S}$, the elements of which admit restrictions to closed subgroups, containing $L$, of $G$. $\mathcal{B}$ will play an important role in Section $V$ too; cf. for this Theorem 2.--In the first part of this section we give the construction of $\mathcal{B}$, in the second we shall obtain a realization, appropriate for our purpose, of a representation quasi-equivalent to $U$ (as above), and most of the third part will be taken up with the construction of a trace on $[R(U)]^{+}$and its direct integral decomposition resp.

2.1. We continue to assume, that $G$ is a connected and simply connected lie group; we recall, that $L=[G, G]$ is a closed, invariant subgroup of type I (cf. Lemma 1.1.1) of $G$. We put $H=G / L$, and denote by $\psi$ the canonical homomorphism from $G$ onto $H$.

If $R$ is the right regular representation of $L$ on the underlying space of $\mathbb{R}=C^{*}(L)$, we have for any $l \in L$ and $v \in \mathbb{R}:\|R(l) v\|=\|v\|$.- Let us consider now the family $\mathcal{B}$ of all those continuous maps $f$ from $G$ into $\mathfrak{Q}$ for which $(1) f(l x)=R(l) f(x)$ for any $l \in L$ and $x \in G$, (2) The map $(H=G / L) x L \mapsto f(x)$ is of a compact support on $H$; we denote by supp $(f)$ 7-742901 Acta mathematica 133. Imprimé le 4 Octobre 1974 
the support of the latter on $H$.-We shall say, that the sequence $\left\{f_{n}\right\} \subset \vec{B}$ converges to $f \in \vec{B}$, if there is a fixed compact subset $C$ of $H$, such that supp $\left(f_{n}\right) \subset C$ for all $n$, and if $f_{n}$ tends to $f$ uniformly on $G$.- Given $f \in \mathcal{L}(G)$, and a fixed element $x$ of $G$, we denote by $\mathcal{F}(f)(x)$ the map $l \mapsto f(l x)=f_{x}(l) \quad(l \in L)$; we have $\mathcal{I}(f)(x) \in \mathcal{L}(L) \subset \mathcal{L}$. The map $x \mapsto \mathfrak{I}(f)(x) \quad(x \in G)$ belongs to $\mathcal{B}$ and thus we can identify $\mathcal{L}(G)$ to a submanifold $B_{0}$ of $B$.-Let us show, that $\mathcal{B}_{0}$ is dense in $\mathcal{B}$. To this end, let $\mathfrak{g}$ be the Lie algebra of $G$ and $\mathfrak{h}$ a complementary subspace to $[\mathfrak{g}, \mathfrak{g}]$ in $\mathfrak{g}$. If $H^{\prime}$ is the image of $\mathfrak{h}$ through the exponential map, $H^{\prime}$ is a closed submanifold of $G$ such that the map $(l, h) \mapsto l h\left(l \in L, h \in H^{\prime}\right)$ from $L \times H^{\prime}$ onto $G$ is a homeomorphism. We denote by $\mathfrak{Q} \otimes \mathcal{C}\left(H^{\prime}\right)$ the linear space of all continuous maps, of a compact support, of $H^{\prime}$ into $\mathfrak{Q}$. Then the map $f \mapsto f \mid H^{\prime}(f \in \mathcal{B})$ is a linear space isomorphism from $\mathcal{B}$ onto $\mathfrak{Q} \otimes \mathcal{L}\left(H^{\prime}\right)$; we shall denote it by $\lambda$. Hence the desired conclusion is implied by the observation that, since $\mathcal{L}(L)$ is dense in $\mathbb{R}$, given $g \in \mathfrak{L} \otimes \mathcal{L}\left(H^{\prime}\right)$, there is a sequence $\left\{g_{n}\right\} \subset \mathcal{L}(G)$, such that the members of $\left\{\lambda\left(\mathcal{I}\left(g_{n}\right)\right)\right\}$ are carried by a fixed compact set $C \subset H^{\prime}$, and such that $\lambda\left(\mathcal{F}\left(g_{n}\right)\right) \rightarrow g$ uniformly on $C$.

Notation 2.1.1. Keeping $H^{\prime}$ (as above) fixed we shall write sometimes $g$ for $\lambda^{-1}(g)$.

Let $d g$ and $d l$ be elements of the right-invariant Haar measure on $G$ and $L$ resp., and $d h$ an invariant measure on $H=G / L$, such that $d g=d h \cdot d l$.-Observe that, if $f \in \mathcal{B}$ and $U \in \operatorname{Rep}(G)$, putting $F(x) \equiv U(f(x)) U(x)$, we have $F(l x) \equiv F(x)(l \in L, x \in G)$. In fact,

$$
U(f(l x))=U(R(l) f(x))=U(f(x))(U(l))^{*}
$$

implying the desired conclusion.-In the following we continue to write mostly $C^{*}(G)=(\mathfrak{S}$.

Lемма 2.1.1. Given $f \in \mathcal{B}$, there is an element $f^{\prime}$ of $C^{*}(G)$, uniquely determined by the property, that for any continuous unitary representation $U$ of $G$ we have

$$
U\left(f^{\prime}\right)=\int_{H} U(f(x)) U(x) \cdot d h
$$

and the map $f \mapsto f^{\prime}(f \in \mathcal{B})$ is a continuous injection of $\boldsymbol{B}$ into $C^{*}(G)$.

Proof. Uniqueness is clear.-In the following we shall denote for a while by $\|f\|_{1}$ the norm of the element $f \in \mathfrak{G}$, while \|\| stands for the norm in $\mathbb{R}=C^{*}(L)$.

For $f \in \mathcal{B}$ let us put $[f]=\int_{H}\|f(x)\| \cdot d h$. We claim, that if $f \in \mathcal{L}(G)$, we have $\|f\|_{1} \leqslant[f]$. To show this we observe, that if $U \in \operatorname{Rep}(G)$, then

$$
U(f)=\int_{H} U(\mathcal{F}(f)(x)) U(x) \cdot d h
$$


In fact

$$
U(f)=\int_{G} f(g) U(g) \cdot d g=\int_{H}\left(\int_{L} f(l x) U(l) \cdot d l\right) U(x) \cdot d h=\int_{H} U(\mathcal{F}(f)(x)) U(x) \cdot d h
$$

Thus $\|U(f)\| \leqslant \int_{H}\|\mathcal{F}(f)(x)\| \cdot d h=[f]$ for any $U \in \operatorname{Rep}(G)$ and hence also $\|f\|_{1} \leqslant[f]$.- - Given $f \in \mathcal{L}(G)=\mathcal{B}_{0} \subset \mathcal{B}$, let us denote by $T^{\prime} f$ the corresponding element of $\left(\mathfrak{S} ; T^{\prime}\right.$ is an injection of $\mathcal{B}_{0}$ into 3 . We claim, that $T^{\prime}$ can be extended to a continuous linear map $T$ from $\vec{B}$ into $\left(\mathcal{S}\right.$. In fact, given $f \in \mathcal{B}$, let us put $\|f\|_{\infty}=\sup _{x \in G}\|f(x)\|$. To arrive at the desired conclusion, it clearly suffices to observe that, for any $f \in \mathcal{B}_{0},\left\|T^{\prime} f\right\|_{1} \leqslant[f] \leqslant h(\operatorname{supp}(f))\|f\|_{\infty}$. For the same reason, by $(+)$ above we can also conclude, that for any $f \in B, U(T f)=$ $\int_{H} U(f(x)) U(x) \cdot d h$. To complete our proof it is therefore enough to show that, if for $f \in \mathcal{B}$ we have $T f=0$, then also $f=0$. In fact, we can take then in the statement of our lemma $f^{\prime}=T f$. - If $T f=0$, replacing above, with $\chi$ arbitrary in $X(G)(\mathrm{A} .8), U$ by $\chi U$ we obtain easily, that $U(f(x)) \equiv 0$ on $G$ for all $U \in \operatorname{Rep}(G)$. Therefore to arrive at our goal it will suffice to prove that if, for a fixed $v \in \Omega$, we have $U(v) \equiv 0$ for any $U \in \operatorname{Rep}(G)$, then $v=0$. Let us choose an arbitrary $\pi \in \hat{L}$ and put $U=\operatorname{ind}_{L \uparrow G} \pi$. We have then $U\left\{L=\int_{H}^{\oplus} h \pi \cdot d h(\mathrm{~A} .11)\right.$, whence $(h \pi)(v) \equiv 0$ almost everywhere in $h \in H$, and thus $v=0$.

Q.e.d.

Remark 2.1.1. By virtue of the previous lemma we can assume from now on, that $(\mathcal{L}(G) \sim) B_{0} \subset \mathcal{B} \subset(\mathfrak{S})\left(=C^{*}(G)\right)$.

Remark 2.1.2. Given a closed, but not necessarily connected subgroup $M$, containing $L$, of $G$, we can form analogously, by replacing above $G$ through $M$, a linear variety $\mathcal{B}_{M} \subset C^{*}(M)$. We shall use Lemma 2.1.1 for this case in section 5 .

Let us put $d\left(a l a^{-1}\right)=\Delta(a) \cdot d l(a \in G)$. We recall (cf. Lemma 1.1.2), that there is a continuous homomorphism $a \mapsto A(a)$ of $G$ into Aut $(\Omega)$ such that if $f \in \mathcal{C}(L) \subset \Omega$, we have $(A(a) f)(l) \equiv f\left(a^{-1} l a\right) / \Delta(a)$. Let us denote by $l \mapsto L(l)$ the left regular representation of $L$ on the underiying space of $\mathbb{2}$. One verifies easily the following relations: $(1) A(l)=L(l) R(l)$, (2) $A(z) L(l)=L\left(z l z^{-1}\right) A(z) \quad(z \in G, l \in L)$, (3) If $\alpha, \beta \in \mathbb{Q}, R(l) \alpha \cdot L(l) \beta=\alpha \cdot \beta \quad(l \in L)$. Let $f$, $g \in B$ and $x \in G$ be fixed, and let us put $G(y) \equiv f\left(x y^{-1}\right) A\left(x y^{-1}\right) g(y)(y \in G)$. By the previous remarks one shows readily, that $G(l y) \equiv G(y)(l \in L, y \in G)$, and thus we can form

$$
\int_{H} f\left(x y^{-1}\right) A\left(x y^{-1}\right) g(y) \cdot d h
$$

LEMM 2.1.2. $\mathfrak{B}$ is a subalgebra of (5) and, if $f, g \in \mathcal{B}, x \mapsto(f g)(x)$ is given by $(+)$ above.

Proof. Denoting by $k(x)$ the expression in $(+)$, a simple computation, using the relations 1-3 above, shows that $k: G \rightarrow \mathfrak{Q}$ belongs to $\boldsymbol{B}$. We shall complete our proof by veri- 
fying, that $U(k)=U(f) U(g)$. We have, since $U(A(a) v)=U(a) U(v) U\left(a^{-1}\right)(a \in G, v \in \mathbb{R})$

$$
\begin{aligned}
U(k(x)) & =\int_{H} U\left(f\left(x y^{-1}\right)\right) U\left(A\left(x y^{-1}\right) g(y)\right) \cdot d h \\
& =\left(\int_{H} U\left(f\left(x y^{-1}\right)\right) U\left(x y^{-1}\right) U(g(y)) U(y) \cdot d h\right) U\left(x^{-1}\right)
\end{aligned}
$$

whence

$$
U(k)=\int_{H} U(k(x)) U(x) \cdot d h=\int_{H}\left(\int_{H} U\left(f\left(x y^{-1}\right)\right) U\left(x y^{-1}\right) U(g(y)) U(y) \cdot d h\right) d h^{\prime}=U(f) U(g)
$$

In the following, whenever necessary to distinguish the * operations on $\mathcal{Q}$ and $\mathbb{S}$ resp., we shall write $v^{s}$ for $v^{*}(v \in \mathbb{R})$.

Lemma 2.1.3. We have $\mathcal{B}^{*}=\mathcal{B}$ and, if $f \in \mathcal{B}, f^{*}(x) \equiv A(x)\left(f\left(x^{-1}\right)\right)^{s} \quad(x \in G)$.

Proof. Let us write $F(x) \equiv A(x)\left(f\left(x^{-1}\right)\right)^{s}(x \in G)$. It is clear, that $F: G \rightarrow \mathfrak{Q}$ is continuous, and that $\left\|F^{\prime}\right\|$ is of a compact support on $H$. Observing, that $A(l) R\left(l^{-1}\right)=L(l)$, $A(x) R\left(x^{-1} l x\right)=R(l) A(x)$ and $(L(l) \alpha)^{s}=R(l)\left(\alpha^{s}\right)(l \in L, x \in G, \alpha \in \mathbb{R})$ it is easily shown, that $F(l x) \equiv R(l) F(x) \quad(l \in L, x \in G)$. Hence $F \in \mathcal{B}$, "and to finish the proof of our lemma, it is enough to show, that $U(F)=(U(f))^{*}$. But

$$
\begin{aligned}
U(F) & =\int_{H} U(F(x)) U(x) \cdot d h=\int_{H} U\left(A(x)\left(f\left(x^{-1}\right)\right)^{s}\right) U(x) \cdot d h=\int_{H} U(x)\left(U\left(f\left(x^{-1}\right)\right)\right)^{*} \cdot d h \\
& =\left(\int_{H} U\left(f\left(x^{-1}\right)\right) U\left(x^{-1}\right) \cdot d h\right)^{*}=\left(\int_{H} U(f(x) U(x) \cdot d h)^{*}=(U(f))^{*}\right.
\end{aligned}
$$

or $U(F)=(U(f))^{*}$, which is the desired conclusion.

Q.e.d.

2.2. The purpose of this second part of section 2 is Lemma 2.2.1. For the proof of Proposition 2 a weaker result would suffice (cf. Remark 2.2.1 below), but in order to speed up things and to keep measure-theoretic considerations, irrelevant in the given context, to a minimum, we prefer to use stronger tools from the theory of $C^{*}$ algebras.

Let $E$ be some orbit of $\tilde{G}$ on $\hat{L}$, which we shall keep fixed. If $\pi$ is an element of $E$, as we saw earlier (cf. (a) of the proof of Lemma 1.2.5) $\tilde{G} / \tilde{G}_{\pi}$ is a connected abelian Lie group, and the natural bijection between $\tilde{G} / \widetilde{G}_{\pi}$ and $E=\tilde{G} \pi$ is a $\tilde{G}$ equivariant homeomor- 
phism. Hence we conclude, that $E$ carries a $\tilde{G}$ invariant Radon measure $\omega$. This being so, we wish to preface Lemma 2.2.1 by a series of observations, which are mostly reminders of results from the theory of $C^{*}$ algebras to be used in the sequel. Below we shall assume, that $\operatorname{dim}(\pi) \equiv+\infty(\pi \in E)$ and shall leave to the reader the modifications necessary to settle the remaining cases.

(1) Since $E$ is locally closed in $\hat{L}$ (cf. (a), proof of Lemma 1.1.5), it is of the form $F^{\prime} \cap O$, where $F$ is closed and $O$ is open in $\hat{L}$. Let us add, that since $E$ is a $\widetilde{G}$ orbit, these can be also assumed to be $\tilde{G}$ invariant. In fact, we can suppose from the start, that $\bar{E}=F$ and replace then $O$ by $\mathrm{U}_{a \in \tilde{G}} a O$.-Hence there are $\tilde{G}$ invariant open sets $\hat{L} \supset O_{1} \supset O_{2}$ such that $E=O_{1}-O_{2}$.

(2) We observe next, that if $O$ is $\tilde{G}$ invariant and open, and $I$ is a closed, two-sided ideal such that $O=\hat{I}$ (cf. loc. cit.), then $I$, too, is $\tilde{G}$ invariant. In fact to this end it is enough to note, that if $F$ is closed in $\hat{L}$, and $a \in \tilde{G}$, then $\operatorname{ker}(a F)=a \cdot \operatorname{ker}(F)$. Let $I_{k}$ be ideals in $\mathcal{Q}$ such that $O_{k}=\hat{I}_{k}(k=1,2)$. Putting $D=I_{1} / I_{2}$, we have $\hat{D}=E$. From what we have just said we conclude, that $I_{k}$ is $\tilde{G}$ invariant, giving rise to an action of $\tilde{G}$ on $D$ and $\hat{D}$; the latter is identical with the induced action of $\tilde{G}$ on $E \subset \hat{L}$.

(3) We recall, that a separable $C^{*}$ algebra $A$ is of a continuous trace (cf. [4], 4.5.4, p. 94] if $\hat{A}$ is Hausdorff and given $\pi_{0} \in \hat{A}$, there is a $p \in A$, such that $\pi(p)$ is a projection of rank one for all $\pi$ in a neighborhood of $\pi_{0}$. We claim, that $D$ (as above) is of a continuous trace. In fact, since $\hat{D}=E$, and $E$ is homeomorphic to the group space of a connected abelian Lie group, $\hat{D}$ is certainly Hausdorff. This implies, that $D$ is postliminaire (cf. e.g. [4], 9.5.2, p. 185). We recall next (cf. [4], 4.4.4. Lemme, p. 91) that any postliminaire $C^{*}$ algebra contains a nonzero closed two-sided ideal of a continuous trace; let $K$ be such with respect to $D$. If $\pi_{0}$ is arbitrary in $\hat{D}$ let $\varrho_{0} \in \hat{K}, a \in \tilde{G}$ be such, that $\pi_{0}=a \varrho_{0}$. By assumption, there is a $p \in K$, such that $\varrho(p)$ is a projection of rank one if $\varrho$ is close to $\varrho_{0}$ inside the open subset $\hat{K}$ of $\hat{D}$. But then, setting $q=a p$, the same will be true of $\pi(q)$ for all $\pi$ in a neighborhood of $\pi_{0}$, completing the proof of our statement.

(4) Let $\mathfrak{H}$ be a Hilbert space such that $\operatorname{dim}(\mathfrak{S})=+\infty, T$ a locally compact space and $A(T)$ the family of all continuous maps, vanishing at infinity, of $T$ in $C(\mathfrak{S})$ (A.13). We can turn $A(T)$ in a $C^{*}$ algebra through pointwise multiplication and by defining, for $f \in A(T)$, its norm through $\sup _{t \in T}\|f(t)\|$. This being so, we recall the following result (cf. [4], 10.9.6. Corollaire, p. 219). Suppose, that $T$ is of a finite dimension, and that $H^{3}(T, Z)=0$. If $A$ is a $C^{*}$ algebra of a continuous trace, such that $\hat{A}=T$, and that any irreducible representation of $A$ is infinite dimensional, then $A$ is ${ }^{*}$ isomorphic to $A(T)$. - We conclude from this, that there is an open subset $O \subset E=D$, such that $\omega(E-O)=0$, and if $D^{\prime}$ is the closed, twosided ideal of $D$ with $\hat{D}^{\prime}=O, D^{\prime}$ is ${ }^{*}$ isomorphic to $A(O)$. In fact, we can certainly choose 
an $O$, as just indicated, such that it be homeomorphic to $\mathbf{R}^{n}$, Then the corresponding $D^{\prime}$ will satisfy all the conditions with respect to $A$ above since we assume, that $\operatorname{dim}(\pi)=+\infty(\pi \in E)$.

(5) Recalling, that $D=I_{1} / I_{2}$ (cf. (2) above), we denote by $I \subset I_{1}$ the complete inverse image of $D^{\prime}$ in $\mathfrak{\Omega}$. We show next, that there is a cross section $\varrho: O \rightarrow \operatorname{Irr}(L)$, such that, with notations as in (4), $A(O)=\left\{\pi \mapsto \varrho_{\pi}(v) ; v \in I\right\}$. In fact, given $w \in D^{\prime}$, let us denote by $\pi \mapsto a(w, \pi)$ the map $O \rightarrow C(\mathfrak{S})$, representing $w$ by virtue of the * isomorphism of (4) between $D^{\prime}$ and $A(O)$. We set $\sigma_{\pi}(w)=a(w, \pi)$, and observe that for each $\pi \in \mathcal{O}$, the map $w \mapsto a(w, \pi)$ is an irreducible * representation of $D^{\prime}$ on $\mathfrak{g}$. We write $\sigma_{\pi}^{\prime}$ for $\sigma_{\pi}$ lifted to $I$. Next we recall (cf. [4], 2.10.4. Proposition, p. 52), that if $A$ is a $C^{*}$ algebra, I a closed two-sided ideal of $A$ and $\sigma$ an irreducible * representation of $I$, there is a unique ${ }^{*}$ representation $\varrho$ of $A$, such that $\varrho \mid I=\sigma$. This being so, we extend $\sigma_{\pi}^{\prime}$ first to $I$ and then to $\Omega$. Denoting by $\varrho_{\pi}$ the representation so obtained, the $\operatorname{map} \pi \mapsto \varrho_{\pi}$ from $O$ into $\operatorname{Irr}(L)$ obviously has the required properties.

(6) Denoting by $U(\mathfrak{S})$ the unitary group of $\mathfrak{g}$ with the strong topology, we are going to show, that the map $(l, \pi) \mapsto \varrho_{\pi}(l)$ from $L \times \mathcal{O}$ into $U(\mathscr{S})$ is continuous (A.14). In fact, the $\operatorname{map}(v, \pi) \mapsto \varrho_{\pi}(v)$ from $I \times \mathcal{O}$ into $C(\mathfrak{S})$ (A.13) (the latter being taken in the uniform topology) is clearly continuous. Next we note, that the right regular representation $R$ of $L$ on $\mathcal{Q}$ leaves any closed two-sided ideal invariant. Let $u, v$ be a fixed pair of elements in $I$. Then $R(l) u \in I_{1} \quad(l \in L)$ and thus $R(l) u \cdot v \in I$. We conclude from this, that the map $f$ of $L \times O$ into $C(\mathfrak{S})$, defined by $\left.f(l, \pi) \equiv \varrho_{\pi}(u) \cdot \varrho_{\pi}(l) \cdot \varrho_{\pi}(v) \equiv \varrho_{\pi}\left(R\left(l^{-1}\right)\right) u \cdot v\right)$ is continuous. Given an element $\pi_{0} \in O$, a compact neighborhood $\mathbf{L}$ of $\pi_{0}$, and a pair, $A, B \in C(\mathfrak{S})$, by (5) we can find $u, v \in I$ such that, on $\mathrm{U}, \varrho_{\pi}(u) \equiv A, \varrho_{\pi}(v) \equiv B$. Hence the desired conclusion follows by observing, that if $\left\{U_{n}\right\}$ and $U$ in $U(\mathfrak{S})$ are such that, for any $A, B \in C(\mathfrak{Y}), A U_{n} B$ tends uniformly to $A U B$, then $U_{n} \rightarrow U$ strongly.

(7) From what we have just seen we conclude, that the map from $\mathfrak{Q} \times O$ into $B(\mathfrak{S})$, sending $(v, \pi)$ into $\varrho_{\pi}(v)$, is also strongly continuous.

(8) We can assume, that there is a $\omega$ measurable field $\left\{\varrho_{\pi} ; \pi \in E\right\}$ such that $\varrho_{\pi}$ is an irreducible representation of class $\pi$ on $\mathfrak{Y}$ and that, on $O, \varrho_{\pi}$ is as above.

We denote by $C_{2}(\mathfrak{S})$ the set of all Hilbert-Schmidt operators on $\mathfrak{Y}$ and, given $A \in B(\mathfrak{S})$, we write $[A]$ for its Hilbert-Schmidt norm $(\leqslant+\infty)$.-As in II.1 we set $H=G / L$ and denote by $d h$ a Haar measure on $H$.-Let us consider now the Hilbert space $\mathfrak{H}_{0}$ corresponding to all those maps $A$ from $G \times E$ into $C_{2}(\mathfrak{L})$, for which $A(l x, \pi) \equiv A(x, \pi)\left(\varrho_{\pi}(l)\right)^{*}$ for any $l \in L, x \in G, \pi \in E$, and such that $[A(x, \pi)]$, as function on $(G / L) \times E$, is $d h \cdot d \omega$ measurable, and

$$
\int_{H \times E}[A(x, \pi)]^{2} d h \cdot d \omega<+\infty .
$$


Given $a \in G$, we denote by $V(a)$ the unitary operator on $\mathfrak{S}_{0}$, which corresponds to the substitution $x \mapsto x a$; the map $a \mapsto V(a)$ is a continuous unitary representation $V$ of $G$ on $\mathfrak{S}_{0}$.Putting $\Pi=\int_{E}^{\oplus} \pi d \omega$ and $m \equiv \operatorname{dim}(\pi)(\pi \in E)$ one sees at once, that $V=m\left(\operatorname{ind}_{L \uparrow G} I I\right)($ A.15).

If $f \in \mathcal{B}$ (cf. 2.1) the function $(x, \pi) \mapsto[\pi(f(x))](\leqslant+\infty)$ is measurable with respect to $d h \cdot d \omega$. In fact, by (7) above, $(x, \pi) \mapsto \varrho_{\pi}(f(x))$ is strongly continuous from $G \times O$ into $B(\mathfrak{S})$, and thus $(x, \pi) \mapsto[\pi(f(x))]$ is lower semicontinuous on $H \times \mathcal{O}$. - We set $(f \in \mathcal{B})$

$$
\|f\|_{\omega}^{2}=\int_{H \times E}[\pi(f(x))]^{2} d h \cdot d \omega \quad(\leqslant+\infty)
$$

write $\mathcal{B}^{\prime}=\left\{f ; f \in \mathcal{B},\|f\|_{\omega}<+\infty\right\}$, and define $\Psi^{\prime}: \mathcal{B}^{\prime} \rightarrow H(V)=\mathscr{H}_{0}$ (A.2) by

$$
\Psi(f)=\left\{\varrho_{\pi}(f(x)) ; x \in G, \pi \in E\right\} .
$$

Lемм а 2.2.1. With notations as above, $\Psi\left(\mathcal{B}^{\prime}\right)$ is dense in $H(V)$.

Proof. Let $H^{\prime}$ be the transversal, modulo $L$, in $G$ of II.1, and let $\eta$ be the image of $d h$ on $H^{\prime}$. We set $\mathfrak{h}=L_{\omega}^{2}(E) \otimes C_{2}(\mathfrak{H})$ and observe, that there is a canonical isomorphism between $H(V)$ and $L_{\eta}^{2}\left(H^{\prime}\right) \otimes \mathfrak{h}$. We also note, that if $\mathcal{B} \in f=v \otimes \varphi$ (cf. Notation 2.1.1), then $\|f\|_{\omega}^{2}=\|\varphi\|^{2}\left(\int_{E}[\pi(v)]^{2} d \omega\right)$. This being so, the desired conclusion follows from the fact (cf. (5) above), that $\mathfrak{h}$ is spanned by its elements of the form $\left\{\varrho_{\pi}(v) ; \pi \in E\right\}(v \in I)$. Q.e.d.

Remark 2.2.1. One could prove Proposition 2 below, goal of section 2, by knowing only, that $\Psi\left(\mathcal{B}^{\prime}\right)$ contains a nonzero element. This is true if we have a $v \in \mathbb{Q}$ such that $\int_{E}[\pi(v)]^{2} \cdot d \omega<+\infty$. In fact, then, for $g \in \mathcal{L}\left(H^{\prime}\right)$, we obtain $f=v \otimes g \in \mathcal{B}^{\prime}$ and $\Psi(f) \neq 0$. One can construct a $v \in \mathbb{Q}$ of the indicated sort as follows. With notations as before, since $D$ is of a continuous trace (cf. (3) above) there is a $w \in D^{+}$such that $\pi \mapsto \operatorname{Tr}(\pi(w)$ ) is a not identically vanishing continuous function on $E$ (cf. [4], 4.5.2, p. 93). We can also assume, that it is zero outside a compact neighborhood of some element $\pi_{0}$ of $E$ (cf. [4], 10.5.6. Corollaire, p. 201). This being, so, it is enough to choose a $v \in \mathbb{Q}$ over $w^{\frac{1}{2}} \in D=I_{1} / I_{2}$ (cf. (2)).

\subsection{Lемм 2.3.1. For $f \in \mathcal{B} \subset C^{*}(G)$ we have $\|f\|_{\omega}=\left\|f^{*}\right\|_{\omega}(\leqslant+\infty)$.}

Proof. We recall (cf. Lemma 2.1.3), that $f^{*} \in \mathcal{B}$ and $f^{*}(x) \equiv A(x)\left(\left(f\left(x^{-1}\right)\right)^{s}\right)$. Therefore, for any $\pi \in E, x \in G$ :

$$
\left[\pi\left(f^{*}(x)\right)\right]=\left[\pi\left(A(x)\left(f\left(x^{-1}\right)\right)\right)\right]=\left[\left(x^{-1} \pi\right)\left(f\left(x^{-1}\right)\right)\right]
$$

and thus, by the $G$ invariance of $d \omega$, 


$$
\begin{aligned}
\left\|f^{*}\right\|_{\omega}^{2} & =\int_{H}\left(\int_{E}\left[\left(x^{-1} \pi\right)\left(f\left(x^{-1}\right)\right)\right]^{2} d \omega\right) d h=\int_{E}\left(\int_{H}\left[\pi\left(f\left(x^{-1}\right)\right)\right]^{2} d h\right) d \omega \\
& =\int_{H \times E}[\pi(f(x))]^{2} d h \cdot d \omega=\|f\|_{\omega}^{2} \quad(\leqslant+\infty)
\end{aligned}
$$

Q.e.d.

We observe, that on (S) there is a canonical conjugation $g \mapsto \bar{g}$, continuing that of $\mathcal{L}(G)$. We put $g^{t}=(\bar{g})^{*}=\left(\overline{g^{*}}\right)(g \in(\mathfrak{S})$.

LEMMA 2.3.2. With notations as in 2.2 and above, if $f \in \mathcal{B}^{\prime}$ and $g \in \vec{B}$ we have $f g \in \mathcal{B}^{\prime}$, and $\Psi(f g)=V\left(g^{t}\right) \Psi(f)$

Proof. We start by showing, that given a compact subset $K \subset H$, there is a constant $C>0$, depending only on $K$, such that if $f \in \mathcal{B}^{\prime}, g \in \mathcal{B}$ satisfy $\operatorname{supp}(f)$, supp $(g) \subset K$, then we have $\|f g\|_{\omega} \leqslant C\|g\|_{\infty}\|f\|_{\omega}$ - - To this end we note first, that if $\mathfrak{h}$ is a Hilbert space then (1) For any $A, B \in B(\mathfrak{h})(\mathrm{A} .13)[A B] \leqslant[A]\|B\|$, (2) If $A: H \rightarrow B(\mathfrak{h})$ is norm continuous and of a compact support, putting $A=\int_{H} A(y) \cdot d y$ we have $[A] \leqslant \int_{H}[A(h)] \cdot d h(\leqslant+\infty)$. Hence, by Lemma 2.1 .2 , if $k=h(\operatorname{supp}(f))$, for any $\pi \in E$ :

$$
\begin{aligned}
{[\pi((f g)(x))] \leqslant } & \int_{H}\left[\pi\left(f\left(x y^{-1}\right)\right)\right]\|g(y)\| d h \\
& \leqslant\|g\|_{\infty}\left(\int_{H}[\pi(f(y))] d h\right) \leqslant k \cdot\|g\|_{\infty}\left(\int_{H}[\pi(f(y))]^{2} d h\right)^{\frac{1}{2}}
\end{aligned}
$$

By virtue of our assumption clearly $\operatorname{supp}(f g) \subset K \cdot K=K^{2}$, and hence

$$
\|f g\|_{\omega}^{2}=\int_{E}\left(\int_{R^{2}}[\pi((f g)(x))]^{2} d h\right) d \omega \leqslant C^{2}\left(\|g\|_{\infty}\right)^{2}\|f\|_{\omega}^{2}
$$

or $\|f g\|_{\omega} \leqslant C\left(\|g\|_{\infty}\right)\|f\|_{\omega}$ where $C=k \cdot\left(h\left(K^{2}\right)\right)^{\frac{1}{2}}$ proving our statement.- If, in our lemma, $g \in \mathcal{L}(G)$, we have $(f g)(x) \equiv \int_{G} g^{t}(a) f(x a) \cdot d a$, whence clearly $f g \in \mathcal{B}^{\prime}$ and $\Psi(f g)=B\left(g^{t}\right) \Psi(f)$.If $g$ is arbitrary in $B$, let $\left\{g_{n}\right\} \subset \mathcal{B}_{0}$ be such, that there is a compact subset $K \subset H$ with $\operatorname{supp}\left(g_{n}\right), \operatorname{supp}(g), \operatorname{supp}(f) \subset K$ and that $\left\|g-g_{n}\right\|_{\infty} \rightarrow 0$. By Lemma 2.1.1 we have then $g_{n} \rightarrow g$ in (S) and hence also $g_{n}^{t} \rightarrow g^{t}$. Therefore $\lim _{n \rightarrow+\infty} \Psi\left(f g_{n}\right)=V\left(g^{t}\right) \Psi(f)$ in $H(V)$.'On the other hand, by what we saw above

$$
\left\|\Psi\left(f \cdot g_{n}\right)-\Psi(f g)\right\|=\left\|f\left(g_{n}-g\right)\right\|_{\omega} \leqslant C\left(\left\|g_{n}-g\right\|_{\infty}\right)\|f\|_{\omega}
$$

and thus finally $\Psi(f g)=\lim _{n \rightarrow+\infty} \Psi\left(f g_{n}\right)=V\left(g^{t}\right) \Psi(f) \quad\left(f \in \mathcal{B}^{\prime}, g \in \mathcal{B}\right)$. 
LEMMA 2.3.3. There is a sequence $\left\{g_{n}\right\} \subset \mathcal{B}^{\prime}$, such that, for any $f \in \mathcal{B}^{\prime}, \Psi\left(f g_{n}\right) \rightarrow \Psi(f)$ in $H(V)$.

Proof. We show first, that if $v \in \mathbb{R}$ is such, that $\int_{E}[\pi(\bar{v})]^{2} d \omega<+\infty$, then for any $\varphi \in \mathcal{L}\left(H^{\prime}\right)$ we have $(v \otimes \varphi)^{t} \in \mathcal{B}^{\prime}$ (cf. Notation 2.1.1). In fact setting, for $w \in \mathfrak{Q}, w^{r}=(\vec{w})^{*}=\overline{w^{*}}$, we get for any $f \in \mathcal{B}: f^{t}(x) \equiv A(x)\left(\left(f\left(x^{-1}\right)\right)^{r}\right)$. Denoting again by $d \eta$ the image of $d h$ on $H^{\prime}$, we obtain thus for $f=v \otimes \varphi$ :

$$
\left\|f^{t}\right\|_{\omega}^{2}=\int_{H}\left|\varphi\left(h^{-1}\right)\right|^{2}\left(\int_{E}\left[\left(h^{-1} \pi\right)(\bar{v})\right]^{2} d \omega\right) d \eta=\|\varphi\|_{2}^{2}\left(\int_{E}[\pi(\bar{v})]^{2} d \omega\right) \quad(\leqslant+\infty)
$$

proving our assertion.-Writing, for $\varphi \in \mathcal{L}\left(H^{\prime}\right), \quad V(\varphi)=\int_{H^{\prime}} \varphi(h) V(h) d \eta$, we have clearly $V(v \otimes \varphi)=V(v) V(\varphi)$. This being said we observe, that to establish our lemma, it is enough to construct a sequence $\left\{v_{n}\right\} \subset \mathbb{Q}$ such that $V\left(v_{n}\right)$ tends strongly to the unit operator and $\int_{E}[\pi(v)]^{2} d \omega<+\infty$. In fact, if $\left\{\varphi_{n}\right\} \subset \mathcal{L}\left(H^{\prime}\right)$ is a sequence, such that $V\left(\varphi_{n}\right) \rightarrow$ unity strongly, we can then set $g_{n} \equiv\left(v_{n} \otimes \varphi_{n}\right)^{t}$ and note, that by Lemma 2.3.2 and by what we said above $\Psi\left(f g_{n}\right)=V\left(v_{n}\right) V\left(\varphi_{n}\right) \Psi(f)$ tends to $\Psi(f)$ in $H(V)$ for any $f \in \mathcal{B}^{\prime}$.-With the notations of 2.2 we have $V=m\left(\operatorname{ind}_{L \uparrow G} \Pi\right)$, where $\Pi=\int_{E}^{\oplus} \pi \cdot d \omega$. Since clearly $a \Pi=\Pi$ (A.6), $V \mid L$ is a multiple of $\Pi$ and hence it is enough to find a sequence $\left\{v_{n}\right\} \subset \mathfrak{R}$, such that $\int_{E}\left[\pi\left(\bar{v}_{n}\right)\right]^{2} \cdot d \omega<+\infty$ and $\underline{\varrho}_{n}\left(v_{n}\right)$ tends to the unity strongly, almost everywhere on $O$ with respect to $d \omega$. But for this it suffices to recall (cf. (4) loc. cit.) that if $A: O \rightarrow C(\mathfrak{S})$ is continuous and of compact support, there is a $w \in \mathbb{R}$, such that $A(\pi) \equiv \varrho_{\pi}(w)(\pi \in O)$. Q.e.d.

Given a unitary (Hilbert) algebra $A$ (cf. [5], p. 70), for any $x \in A$ we denote by $V_{x}$ the bounded operator on the completion of the underlying pre-Hilbert space of $A$, uniquely determined by $V_{x} y=y x(y \in A)$. We recall, that the right ring $\mathfrak{V}(A)$ of $A$ is the $v$. Neumann algebra generated by the operators $\left\{V_{x} ; x \in A\right\}$.

LEMMA 2.3.4. With the notations of Lemma 2.2.1, we can define the structure of a unitary algebra $\mathcal{E}$ on $\Psi\left(\mathcal{B}^{\prime}\right)$, such that $R(V)=\mathfrak{V}(\mathcal{E})$ and $V(\mathcal{B}) \supset\left\{V_{x} ; x \in \mathcal{E}\right\}$.

Proof. Given $f, g \in \mathcal{B}^{\prime}$ let us set $(f, g)=(\Psi(f), \Psi(g))$. By Lemma 2.3.1, $\left(\boldsymbol{B}^{\prime}\right)^{*}=\boldsymbol{B}^{\prime}$ and $(f, g)=\left(g^{*}, f^{*}\right)$. If $h \in \mathcal{B}^{\prime}$, Lemma 2.3 .2 implies, that

$$
\begin{aligned}
(f h, g)=(\Psi(f h), \Psi(g))=\left(V\left(h^{t}\right) \Psi^{2}(f), \Psi(g)\right) & =\left(\Psi(f), V\left(\left(h^{*}\right)^{t}\right) \Psi(g)\right) \\
& =\left(\Psi(f), \Psi\left(f h^{*}\right)\right)=\left(f, g h^{*}\right)
\end{aligned}
$$

Let us consider now the factor algebra $\mathcal{E}=B^{\prime} / \operatorname{ker}(\Psi) \sim \Psi^{\prime}\left(\mathcal{B}^{\prime}\right)$. The map $f \mapsto f^{*}$ of $\mathcal{B}^{\prime}$ onto itself gives rise to an involutive antiautomorphism of $\mathcal{E}$ (to be denoted in the same manner), such that with the inner product (,) induced by that of $H(V)$ we have for any 
$x, y, z \in \mathcal{E}:(x y, z)=\left(x, x y^{*}\right)$ and $(x, y)=\left(y^{*}, x^{*}\right)$.-By Lemma 2.3.3, the set $\{x y ; x, y \in \mathcal{E}\}$ is dense in $\mathcal{E}$. If $x, y \in \mathcal{E}$, and $x=\Psi(f)\left(f \in \mathcal{B}^{\prime}\right)$, we have $V_{x} y=V\left(f^{t}\right) y$; hence, for any fixed $x \in \mathcal{E}$ the map $y \mapsto y x$ of $\mathcal{E}$ into itself is continuous, completing the proof, that $\mathcal{E}$ constitutes a unitary algebra.-We have just seen, that $V(\mathcal{B}) \supset\left\{V_{x} ; x \in \mathcal{E}\right\}$ whence, using $\overline{\mathcal{B}}=\mathbb{B}$, $\mathcal{B}^{\prime} \cdot \mathcal{B} \subset \mathcal{B}^{\prime}$ and the proof of Lemma 2.3.3 (or quite simply the commutation theorem, ef. loc. cit. p. 71 , bottom) we get $R(V)=\mathfrak{V}(\mathcal{E})$.

Q.e.d.

Lemma 2.3.5. Let $O$ be an orbit of $\Sigma$ on $\mathfrak{A}$ (cf. Lemma 1.1.6). There is a positive, $G$ invariant Radon measure on $\mathrm{O}$, uniquely determined up to a positive constant factor.

Proof. With notations as in Lemma 1.1.6 we assume, that $O=\bar{G}$, closure being taken in $F$. Let us set $A=N / N_{\ell}$ and recall (cf. (e), proof of Lemma 1.1.5), that the map $i: A \rightarrow F$ defined by $i\left(a N_{\varrho}\right)=a \underline{a}(a \in N)$ is an $N$ equivariant homeomorphism. If $A^{\prime}$ is the image of $G$ in $A$, we have $A=\bar{A}^{\prime}$ and $i(A)=O$. Hence if $\eta$ is a Haar measure on $A, i_{*}(\eta)=\boldsymbol{\nu}$ is a positive, $G$ invariant Radon measure on $O$. Conversely, let $\mu$ be such a measure and suppose, that $\mu=i_{*}\left(\eta^{\prime}\right)$. Then $\eta^{\prime}$ is a positive, $A^{\prime}$ invariant Radon measure on $A$ and hence there is a constant $c>0$ such that $\eta^{\prime}=c \eta$, and therefore also $\mu=c v$.

Q.e.d.

Let $J$ be a fixed element of Prim $(G)$; below we shall write $E=E(J), F=F(J), K=$ $K(J)$ (cf. Notation 1.1.3) and set $O=A(J)(\in F / \Sigma$; cf. Proposition 1).

As already mentioned earlier (cf. the introduction to section 2), we shall say, that $T \in \operatorname{Rep}(G)$ (A.4) is a representation with trace, if $R(T)$ (A.2) carries a faithful, normal, semifinite trace $\Phi$, such that the set $\left\{T(a) ; a \in \mathfrak{B S}^{+}, \Phi(T(a))<+\infty\right\}$ generates $R(T)$. If in addition, $T \in \mathrm{Fac}(G)$, we shall call $T$ a normal representation.

Notation 2.3.1. We shall write Facn $(G)$ for the subset, composed of all normal representations, of Fac $(G)$.

We recall (cf. (a), proof of Lemma 1.2.1), that there is a Borel cross section $\varrho: F \rightarrow \operatorname{Irr}(K)$.

Lемма 2.3.6. With notations as above, let $\mu$ be a positive $G$ invariant Radon measure on $O$ (ct. Lemma 2.3.5), and let us put

$$
T=\operatorname{ind}_{x \uparrow G}\left(\int_{0}^{\ominus} \varrho(\xi) \cdot d \mu(\xi)\right)
$$

Then $T$ is a normal representation with $\operatorname{ker}(T)=J$, and there is a $\varphi \in \mathcal{B}^{+}\left(=\mathcal{B} \cap\left(S^{+}\right)\right.$, such that $0<T(\varphi)$ and $T(\varphi)$ is of a finite trace. 
Proof. We infer at once from (a), proof of Lemma 1.2.1, that $T \in$ Fac $(G)$, and $\operatorname{ker}(T)=$ $J$. Hence, in order to establish our lemma, what we have to show here is, that $T$ is normal.

(a) Let again $\omega$ be a $\widetilde{G}$ invariant Radon measure on $E$ (cf. II.2). Fixing a Borel cross section $\pi: E \rightarrow \operatorname{Irr}(L)$, let us set $\Pi=\int_{E}^{\oplus} \pi(\eta) \cdot d \omega(\eta)$. Let $\tau^{\prime}$ be a Haar measure on $A=$ $N / N_{\varrho}$ (cf. Lemma 2.3.5) and $\tau=i_{*}\left(\tau^{\prime}\right)$, Putting $Y=\int_{F}^{\oplus} \varrho(\zeta) \cdot d \tau(\zeta)$ we claim, that $Y=$ ind $_{L \uparrow K} \Pi$. In fact, let us define $P: F \rightarrow E$ by $P(\varrho)=\varrho \mid L(\varrho \in F)$; clearly $P$ is continuous. Furthermore, if $\sigma$ is some element of $F, \pi=P(\sigma)$ and $₹$ the annihilator of $L$ in $X(K)$ (cf. (c), proof of Lemma 1.1.5), the map $\varphi \mapsto \varphi \sigma$ is a homeomorphism between $¥$ and $P^{-1}(\pi)$. Thus we can write $\tau=\int_{E}^{\oplus} \tau_{\pi} d \omega(\pi)$, where $\tau_{\pi}$ is $\mathcal{F}$ invariant on $F$ and carried by $P^{-1}(\pi)(\pi \in E)$. We recall, that if $Y(\eta)=\int_{F}^{\ominus} \varrho(\zeta) d \tau_{\eta}(\zeta)$, then $Y(\eta)=$ ind $_{L \uparrow K} \pi(\eta)$ (cf. [20], Lemma 1, p. 325). Moreover (cf. [17], Theorem 2.11, p. 204 and [16], Theorem 10.1, p. 123) the field $\eta \mapsto Y(\eta)$ is $\omega$ measurable and

$$
Y=\int_{F}^{\oplus} \varrho(\xi) \cdot d \tau(\xi)=\int_{E}^{\oplus} Y(\eta) \cdot d \omega(\eta)=\int_{E}^{\oplus}(\underset{L \uparrow K}{\operatorname{ind}} \pi(\eta)) \cdot d \omega(\eta)=\operatorname{ind}_{L \uparrow K} \Pi
$$

proving our assertion.

(b) We write $\Lambda=F / \Sigma$ and, if $\lambda \in \Lambda$, we denote by $O_{\lambda}$ the corresponding $\Sigma$ orbit. By the proof of Lemma 2.3.5, there is a Radon measure $\nu$ on $\Lambda$, and for each $\lambda \in \Lambda$, a $G$ invariant Radon measure $\tau_{\lambda}$, carried by $O_{\lambda}$, on $F$, such that $\tau=\int_{\Lambda} \tau_{\lambda} d \nu(\lambda)$. Therefore, putting $Z(\lambda)=\int_{H^{\prime}}^{\oplus} \varrho(\zeta) \cdot d \tau_{\lambda}(\zeta)$ we obtain, similarly as in (a), that

$$
Y=\int_{F}^{\oplus} \varrho(\xi) \cdot d \tau(\xi)=\int_{\Lambda}^{\oplus} Z(\lambda) \cdot d \nu(\lambda)
$$

Let us write now $U=\operatorname{ind}_{L \uparrow G} \Pi$ and $U(\lambda)=\operatorname{ind}_{K \uparrow G} Z(\lambda)$; then by $Y=\operatorname{ind}_{L \uparrow K} \Pi$ (cf. (a)) we conclude, that

$$
U=\operatorname{ind}_{K \uparrow G} Y=\int_{\Lambda}^{\oplus} U(\lambda) \cdot d \nu(\lambda)
$$

Let us note, that by virtue of our construction, there is a $\lambda_{1} \in \Lambda$ such that $U\left(\lambda_{1}\right)=T$ (the latter as in the statement of our lemma).

(c) We claim, that the decomposition $(+)$ is central. This we infer from the following result of E. Effros (implied by [8], Theorem 1.10, p. 91). Let $(Z, \alpha)$ be a standard measure space, $\zeta \mapsto T(\zeta)$ an $\alpha$-measurable field of factor representations on $Z$, such that $\operatorname{ker}\left(T\left(\zeta_{1}\right)\right)=$ $\operatorname{ker}\left(T\left(\zeta_{2}\right)\right)$ only for $\zeta_{1}=\zeta_{2}$. Then the direct integral $\int_{Z}^{\oplus} T(\zeta) \cdot d \alpha(\zeta)$ is central.-To obtain the desired conclusion, it is enough to recall, that $\operatorname{ker}(U(\lambda)) \equiv J(\varrho)\left(\varrho \in O_{\lambda}\right)$ and thus, by Lemma 1.1.9, $\lambda_{1} \neq \lambda_{2}$ implies $\operatorname{ker}\left(U\left(\lambda_{1}\right)\right) \neq \operatorname{ker}\left(U\left(\lambda_{2}\right)\right)$.

(d) We observe next, that if there is a $\lambda_{0} \in \Lambda$ such that $U\left(\lambda_{0}\right) \in$ Facn $(G)$ (cf. Notation 
2.3.1 above), then we have $U(\lambda) \in$ Fac $(G)$ for all $\lambda \in \Lambda$. To see this, let us form the direct product $\tilde{N}=\tilde{G} \times X(G)$. If $a=(b, \chi) \in \tilde{N}$ and $T \in \operatorname{Rep}(G)$, we set $(a T)(g) \equiv \chi(g) T\left(b^{-1} g b\right)$ $(g \in G)$, in which fashion we get a left action of $\tilde{N}^{2}$ on $\operatorname{Rep}(G)$. Similarly, we let $N$ act on the left on $\operatorname{Rep}(K)$. -We define $t: X(G) \rightarrow \mathcal{F}$ by $t(\chi) \equiv \chi \mid K$ and write $(a=(b, \chi) \in \widetilde{N}): j(a)=$ $(b, t(\chi)) \in N$. Let us observe, that since $N$ acts on $F$ as an abelian group, it preserves $\Sigma$ orbits and hence acts transitively on $\Lambda=F / \Sigma$. This being so we claim, that $(a U)(\lambda)=$ $U(j(a) \lambda)(\lambda \in \Lambda)$. In fact, since $U(\lambda)=$ ind $_{K \uparrow G} Z(\lambda)$ (cf. (b)) we have clearly $a U(\lambda)=$ $\operatorname{ind}_{K \uparrow G}(j(a) Z(\lambda))$. Putting $b=j(a)$ and $b \lambda=\varkappa$, the image of $d \tau_{\lambda}$, under the action of $b$ on $F$, is a multiple of $d \tau_{x}$. In this fashion, in the sense of unitary equivalence

$$
b Z(\lambda)=\int_{F}^{\oplus} b \varrho(\xi) \cdot d \tau_{\lambda}(\xi)=\int_{F}^{\oplus} \varrho(b \xi) \cdot d \tau_{\lambda}(\xi)=\int_{F}^{\oplus} \varrho(\xi) \cdot d \tau_{\varkappa}(\xi)=Z(x)
$$

Hence finally

$$
a U(\lambda)=\operatorname{ind}_{K \uparrow G}(j(a) Z(\lambda))=\operatorname{ind}_{K \uparrow G} Z(j(a) \lambda)=U(j(a) \lambda)
$$

proving our statement.-Assume now, that $\lambda_{0}$ is as at the start of (d). Given $\lambda \in \Lambda$, by virtue of what we have just seen, there is an $a \in \tilde{N}$, such that $U(\lambda)=a U\left(\lambda_{0}\right)$. On the other hand, letting $\tilde{N}$ act on $(B)=C^{*}(G)$ as in Lemma 1.1.2, there is an $a^{\prime} \in \tilde{N}$ with $\left(a U\left(\lambda_{0}\right)\right)(f) \equiv$ $U\left(\lambda_{0}\right)\left(a^{\prime} f\right)\left(f \in(\mathbb{S})\right.$. Hence if $U\left(\lambda_{0}\right) \in$ Facn $(G)$, we have also $U(\lambda) \in$ Facn $(G)$ for all $\lambda \in \Lambda$, as claimed above.

(e) From here we complete the proof of Lemma 2.3.6 as follows. Replacing in Lemma 2.3.4 $E \in \hat{L} / \widetilde{G}$ through $\bar{E} \in \hat{L} / \widetilde{G}$ (A.15) we observe, that $V=m \cdot \operatorname{ind}_{L \uparrow G} \Pi=m \cdot U$. On the other hand we showed loc. cit., that there is a unitary algebra $\mathcal{E}$, such that $\mathfrak{V}(\mathcal{E})=R(V)$ and $V(\mathcal{B}) \supset\left\{V_{x} ; x \in \mathcal{E}\right\}$. Therefore, if $\Phi$ is the natural trace on $[\mathcal{V}(\mathcal{E})]^{+}$(cf. [5], Definition 2, p. 88), $V$ is a representation with the trace $\Phi$. If $\Phi^{\prime}$ corresponds to $\Phi$ on $[R(U)]^{+}, U$ will then be a representation with the trace $\Phi^{\prime}$.- Since the decomposition $U=\int_{\Lambda}^{\oplus} U(\lambda) \cdot d v(\lambda)$ (cf. (+) in (b)) is, by (c), central, we have also (dropping the prime) $\Phi=\int_{\Lambda}^{\oplus} \Phi_{\lambda} d \nu(\lambda)$, where $\Phi_{\lambda}$ is a faithful, normal, semifinite trace on $[R(U(\lambda))]^{+}$such that, if $A \in[R(U)]^{+}$and $A=$ $\int_{\Lambda}^{\oplus} A(\lambda) \cdot d v(\lambda)$, we get $\Phi(A)=\int_{\Lambda} \Phi_{\lambda}(A(\lambda)) \cdot d v(\lambda)(\leqslant+\infty)$ (cf. [5], Théorème 2, (iii), p. 200). Let $a \in \mathcal{B}^{+}=\mathcal{B} \cap \mathfrak{B}^{+}$be such, that $0<\Phi(U(a))<+\infty$; for instance, take $b \in \mathcal{B}^{\prime}$ with $U\left(b^{t}\right) \neq 0$ and set $a=b^{t} \cdot\left(b^{t}\right)^{*}$. What we have just seen evidently implies, that there is a $\delta \in \Lambda$ satisfying $0<\Phi_{\delta}(U(\delta)(a))<+\infty$, and therefore $U(\delta) \in$ Facn $(G)$ (cf. [4], 6.7.2. Proposition, p. 127). Hence we can apply (d) above with $\lambda_{0}=\delta$ and conclude, that $U(\lambda) \in$ Facn $(G)$ for all $\lambda \in \Lambda$, and thus, in particular (cf. the end of (b)) $T=U\left(\lambda_{1}\right) \in$ Facn $(G)$.--Let us show finally, that there is a $\varphi \in \mathcal{B}^{+}=\mathcal{B} \cap \mathscr{G}^{+}$, such that $0<T(\varphi)$ and that $T(\varphi)$ is of a finite trace. By what we have just seen we can find $V \in$ Facn $(G), \alpha \in \tilde{N}$ and $a \in B^{+}$such that $T(f) \equiv$ 
$V(\alpha(f))(f \in(B)$ and that $V(a)$ is of a finite positive trace. Hence it suffices to verify, that for any $\beta \in \tilde{N}$ we have $\beta\left(\mathcal{B}^{+}\right) \subseteq \mathcal{B}^{+}$; in fact, we can take then $\varphi=\left(\alpha^{-1}\right)(a)$. Clearly, it is enough to show, that $\beta(\mathcal{B}) \subseteq \mathcal{B}$. Suppose, that $\beta=(b, \chi)$, and let us denote by $A(b)$ the automorphism of $\mathfrak{Q}$ corresponding to $b \in G$ (cf. Lemma 1.1.2). For a fixed $f \in B$ we set $F(x)=$ $\chi(x) \cdot A(b)\left(\left(f\left(b^{-1} x b\right)\right)(x \in G)\right.$. By observing, that $A(b) R\left(b^{-1} l b\right)=R(l) A(b)$ one verifies easily, that $x \mapsto F(x)$ belongs to $\mathcal{B}$. We denote by $\boldsymbol{F}$ the corresponding element of $(S)$ (cf. Lemma 2.1.1) and claim, that $F=\beta$ f. If $U \in \operatorname{Rep}(G)$, we have $U(\beta F)=\left(\chi\left(b^{-1} U\right)\right)(f)($ A.6, A.8). On the other hand, since $U(A(b) v) \equiv\left(b^{-1} U\right)(v)$ and $(\chi U)(v) \equiv U(v)(v \in \mathfrak{L})$

$$
\begin{aligned}
U(F) & =\int_{H} U(F(x)) U(x) \cdot d h=\int_{H} U\left(A(b)\left(f\left(b^{-1} x b\right)\right)\right)(\chi U)(x) \cdot d h \\
& =\int_{H}\left(\chi b^{-1} U\right)(f(x))\left(\chi b^{-1} U\right)(f(x)) \cdot d h=\left(\chi b^{-1} U\right)(f)=U(\beta f)
\end{aligned}
$$

completing the proof of our lemma.

Q.e.d.

We sum up the previous discussion in the following

Proposition 2. Suppose, that $G$ is a connected and simply connected Lie group, and $J$ some element of Prim $(G)$. There is a normal representation the kernel of which is $J$.

Proof. This follows at once from Lemma 2.3.6.

Q.e.d.

Remark 2.3.1. The following observation will be useful in Section 5 (for the reasonings employed below cf. [4] 6.6, p. 125). Suppose, that $V_{j} \in \mathrm{Fac}(G), \Phi_{j}$ is a normal trace on $\left[R\left(V_{3}\right)\right]^{+}$such that $\Phi_{1}\left(V_{1}(a)\right) \equiv \Phi_{2}\left(V_{2}(a)\right)\left(a \in B^{+}\right)$and that, for some $b \in \mathcal{B}^{+}$, we have $0<$ $\Phi_{2}\left(V_{2}(b)\right)<+\infty$. Then $V_{1}$ and $V_{2}$ are quasi-equivalent.-Let us start by noting that, if $\mathfrak{m}_{j}$ is the ideal of $\left(\mathfrak{S}\right.$ corresponding to $V_{j}$ and $\mathfrak{n}_{j}=\mathfrak{m}_{j}^{\frac{1}{2}}$, we have $\mathfrak{n}_{1} \cap \mathcal{B}=\mathfrak{n}_{2} \cap \mathcal{B}$ (cf. [4], 6.1.2, p. 112). In fact, $a \in\left(B S\right.$ belongs to $\mathfrak{n}_{1} \cap \mathcal{B}$ if and only if $a \in \mathcal{B}$ and $\Phi_{1}\left(V_{1}\left(a^{*} \cdot a\right)\right)<+\infty$; but then also $a \in \mathfrak{n}_{2} \cap \mathcal{B}$, and conversely. Let us put $\mathcal{B}_{1}=\mathfrak{n}_{j} \cap \mathcal{B}$. If $A_{j}$ is the unitary algebra corresponding to $\mathrm{m}_{j}^{\frac{1}{2}}$, the image of $\mathcal{B}_{1}$ in $A_{j}$ is dense $(j=1,2)$. In fact, if $A=A_{1}$ (say), $\mathfrak{H}$ is the completion of $A$ and $\mathfrak{h}$ the closure of the image of $\mathcal{B}_{1}$ in $\mathfrak{H}$, then $\mathfrak{h}$ is nonzero, and invariant under the left and right actions by elements of $\mathcal{B}$. Hence, since $V_{1} \in$ Fac $(G)$, we have $\mathfrak{h}=\mathfrak{S}$. Summing up, there is an isometry from the completion of $A_{1}$ onto that of $A_{2}$, making correspond to each other left actions of $B$. Thus $V_{1}$ is quasi-equivalent to $V_{2}$. Q.e.d.

Remark 2.3.2. Let $G$ be as above. We write $\mathcal{L}_{G}$ for its left regular representation on the 
Hilbert space $L^{2}(G)$ of all square integrable functions with respect to a right invariant Haar measure on $G$, and set $R\left(\mathcal{L}_{G}\right)=\mathcal{U}(G)$; then $\mathcal{U}(G)$ is the left ring of $G$. The purpose of the following observations is to sketch a proof, on the basis of our preceeding considerations, that $\mathcal{U}(G)$ is semifinite. We recall, that due to a theorem of J. Dixmier (cf. [6], Théorème, p. 423) the analogous statement is valid for any separable, locally compact and connected group.

Let $\mathcal{L}_{L}$ be the left regular representation of $L$; we have $\mathcal{L}_{G}=\operatorname{ind}{ }_{L \uparrow G} \mathcal{L}_{L}$. We note, incidentally, that $L=[G, G]$ is unimodular; in fact, if $L=\exp (\delta)(A .1)$, the radical of $\delta$ is nilpotent. Let $\mu$ be the Plancherel measure of $L$ and $\pi: \hat{L} \rightarrow \operatorname{Irr}(L)$ a Borel cross-section. Putting $\Pi=\int_{\hat{L}}^{\ominus} \pi(\zeta) \cdot d \mu(\zeta)$, we have $\mathcal{L}_{L} \approx \Pi$ (A.5), and hence, if $V=$ ind $_{L \uparrow G} \Pi$, also $\mathcal{L}_{G} \approx V$. In this fashion it is enough to establish, that $R(V)$ is semifinite. - $\tilde{G}$ acts on $\hat{L}$ as an abelian group, such that $S=\hat{L} / \tilde{G}$ is countably separated (cf. Lemma 1.1.3). Hence we can write $\mu=\int_{S} \mu_{s} \cdot d v(s)$ where, up to a set of $\nu$ measure zero, $\mu_{s}$ is $\sigma$-finite, is carried by $s \in S$ and, if $a \mu=\delta(a) \mu \quad(a \in \tilde{G})$, also $a \mu_{s}=\delta(a) \mu_{s}$. Therefore, in particular, $\mu_{s}$ is equivalent to a $\tilde{G}$ invariant Radon measure on $s$. Let us set $\Pi(s)=\int_{\hat{L}}^{\oplus} \pi(\zeta) \cdot d \mu_{s}(\zeta)$ and $V(s)=\operatorname{ind}_{L \uparrow G} \Pi(s)$. It is implied by Lemma 2.3.4 that, if $s$ does not belong to a set of $v$ measure zero, $V(s)$ is a representation with trace and thus, in particular, $R(V(s))$ is semifinite. Since $\Pi=$ $\int_{S}^{\oplus} \Pi(s) \cdot d v(s)$, we have also $V=\int_{S}^{\oplus} V(s) \cdot d v(s)$. The latter decomposition is central; in fact, to see this, it suffices to note, that $V(s) \mid L \approx \Pi(s)$, and that $\int_{S}^{\oplus} \Pi(s) \cdot d \nu(s)$ is certainly central. Thus finally, the semifiniteness of $R(V)$ is implied by [5], Corollaire 2, p. 206.

Q.e.d.

\section{§ 3}

The purpose of the next two sections is to show, that any normal representation, up to quasi-equivalence, is uniquely determined by its kernel (cf. Proposition 4, section 4). In the present section 3 we shall treat separately a special case of interest, where the desired conclusion is implied by standard results.

LEMMA 3.1. The following properties of $J \in \operatorname{Prim}(G)$ are equivalent: (1) If $V \in$ Fac $(G)$ and $\operatorname{ker}(V)=J$, then $V$ is of type $I,(2)$ There is an irreducible normal representation of kernel $J$.

Proof. $(1) \Rightarrow(2)$ By Proposition 2, there is a $U \in$ Facn $(G)$ with $\operatorname{ker}(U)=J$. Our assumption implies, that $U$ is of type $I$; hence, to satisfy (2) it is enough to take $W \in \operatorname{Irr}(G)$ such that $U \approx W(\mathrm{~A} .5)$.

$(2) \Rightarrow(1)$ Here we employ the following well-known assertion (implied by [4], 4.1.10. 
Corollaire). If $A$ is a $C^{*}$ algebra and $\pi \in \operatorname{Irr}(A)$ is normal, then $\operatorname{ker}(\pi)=\operatorname{ker}\left(\pi^{\prime}\right)\left(\pi^{\prime} \in \operatorname{Irr}(A)\right)$ implies $\pi \sim \pi^{\prime}$ (A.5). By virtue of this we obtain the desired conclusion using a reasoning due to J. Dixmier (cf. [3], Remarque, p. 100). Let us assume, that $V \in$ Fac $(G)$ is such, that $\operatorname{ker}(V)=J$. Suppose, that $V=\int_{X}^{\oplus} V(x) \cdot d \mu(x)$ is a decomposition of $V$ into a direct integral, over the standard measure space $(X, \mu)$, of irreducible representations. Then (cf. loc. cit.) $\operatorname{ker}(V(x)) \equiv \operatorname{ker}(V)=J$ almost everywhere with respect to $\mu$. By assumption, there is an irreducible normal representation $W$ with $\operatorname{ker}(W)=J$. What we said above implies, that $V(x) \sim W$ up to a set of $\mu$ measure zero. Hence $V \approx W$ and $V$ is of type $I . \quad$ Q.e.d.

We shall say, that $J \in \operatorname{Prim}(G)$ is of type $I$, if $J$ satisfies one of the two conditions of Lemma 3.1. Incidentally, from what we saw above is clear, that if $J$ is such, any two normal representations of kernel $J$ are quasi-equivalent.-Our next objective is a characterization of such ideals (ef. Proposition 3 below).

We observe, that if $E \in \hat{L} / G$ is fixed, and $\varrho \in F(E), G_{\varrho}$ (A.9) does not change; hence we may write $G_{\varrho} \equiv \mathfrak{U}(E)$. This being so we recall (cf. [22], Lemma 3.7, p. 484) that $K(E)(G(E))_{0}$ (cf. Notation 1.1.1) is an open subgroup of $\mathfrak{H}(E)$. Denoting its index by $n(E)(\leqslant+\infty)$, if $\varrho \in F(E), T(\varrho)=\operatorname{ind}_{K(E) \uparrow G} \varrho \in \mathrm{Fac}(G)$ (cf. Lemma 1.1.4, (i)) is of type $\mathrm{I}$ if and only if $n(E)<+\infty$. - Given $J \in \operatorname{Prim}(G)$ we set $n(J)=n(E(J))$ (cf. Notation 1.1.3).

If $G$ is solvable, the integer $n(J)$ can be described as follows. In this case we have a canonical bijection between points of Prim $(G)$ and generalized orbits (cf. [24], III.6, p. 85 and loc. cit. Theorem 1, or Section VI below resp.). Let $J \in \operatorname{Prim}(G)$ and $O$ be the corresponding generalized orbit. Let $\mathfrak{g}$ be the Lie algebra of $G$, and $g$ an arbitrary element of the projection of $O$ into $g^{\prime}$. Then $n(J)$ is equal to the index of the reduced stabilizer $\bar{G}_{g}$ (cf. [24], 3.3, p. 83) in $G_{g}$.

We assume now again, that $G$ is an arbitrary connected and simply connected Lie group. Given $J \in \operatorname{Prim}(G)$ we recall, that $A(J)=\{\varrho ; \varrho \in \mathfrak{A}, J(\varrho)=J\}$ (cf. Proposition 1). Observe, that $G$ acts on $\mathcal{A}(J)$ as an abelian group (cf. e.g. Lemma 1.1.6).

Proposition 3. $J \in \operatorname{Prim}(G)$ is of type $\mathrm{I}$ if and only if (1) $G$ acts transitively on $\mathcal{A}(J),(2) n(J)$ is finite.

Proof. Let us assume first, that $J$ is of type I. We form, as in Lemma 2.3.6, the representation

$$
T=\operatorname{ind}_{K(J) \uparrow G}\left(\int_{A(J)}^{\oplus} \varrho d \mu(\varrho)\right)=\int_{A(J)}^{\oplus} T(\varrho) \cdot d \mu(\varrho)
$$

We know (cf. loc. cit.) that $T \in \mathrm{Fac}(G)$ and $\operatorname{ker}(T)=J$. Since $T\left(\varrho_{1}\right) \approx T\left(\varrho_{2}\right)$ if and 
only if $G \varrho_{1}=G \varrho_{2}$ (cf. Lemma 1.1.4, (ii)) by a routine reasoning (cf. e.g. [22], Lemma 2.3.2, p. 550) we conclude, that if $T$ is of type $\mathrm{I}, \mathcal{A}(J)$ must be a $G$ orbit. But then, for any $\varrho \in \mathcal{A}(J), T \approx T(\varrho)$; hence $T(\varrho)$ is itself of type $\mathrm{I}$, implying $n(J)<+\infty$.-Conversely, if $J \in \operatorname{Prim}(G)$ satisfies the two conditions of our lemma, then $T(\varrho)$ is of type $I$ for $\varrho \in \mathcal{A}(J)$ and, as above, Facn $(G) \in T \approx T(\varrho)$. In other words, $T(\varrho)(\varrho \in \mathcal{A}(J))$ is normal and of type I which, by our definition, yields the desired conclusion.

Q.e.d.

We shall discuss the implications of assuming only, that $\mathcal{A}(J)$ is a $G$ orbit, in Proposition 5.4 .3 below.

\section{§4}

The main objective in this section is to show, that if $G$ is connected and simply connected, $V_{j} \in \operatorname{Facn}(G)$ and $\operatorname{ker}\left(V_{1}\right)=\operatorname{ker}\left(V_{2}\right)=J$ (say), then $V_{1} \approx V_{2}$ (cf. Proposition 4). This implies easily the analogous result for a not necessarily simply connected, connected group (cf. Theorem 1). By what we saw in section 3, we can assume, that $J$ is not of type $\mathrm{I}$.

4.1. We put $E=E(J)$, fix an element $\pi \in E$ and recall the following (for more details, cf. [22], start of Section 3, Chapter I, p. 480-481). Let $\pi^{e}$ be a projective extension of $\pi$ to $G_{\pi}$, giving rise to a continuous cocycle $\alpha\left(\alpha\left(g_{1} l_{1}, g_{2} l_{2}\right) \equiv \alpha\left(g_{1}, g_{2}\right) ; g_{1}, g_{2} \in G_{\pi} ; l_{1}, l_{2} \in L\right)$. We put $G_{\pi}^{e}=G^{e}$ and note, that $G^{e}$ can be realized as the set $\left\{(a, u) ; a \in G_{\pi}, u \in \mathbf{T}\right\}$ with the multiplication $(a, u)(b, v)=(a b, u v \alpha(a, b))$ and the product topology. The subset $\{(l, 1)$; $l \in L\}$, to be denoted again by $L$, is a closed, invariant subgroup of $G^{e}$. Let us set $\mathbf{M}_{\pi}=G^{e} / L$ and denote by $\Phi$ the canonical homomorphism $G^{e} \rightarrow \mathbf{M}_{\pi}$. Since $G$ is connected and simply connected, we have

$$
\mathbf{l} \longrightarrow \mathrm{T} \stackrel{\text { central }}{\longrightarrow} \mathbf{M}_{\pi} \longrightarrow Z \longrightarrow 1
$$

where $Z$ is direct product of a vector group with a free abelian group. Below we shall put $\Gamma=\mathbf{M}_{\pi}$.

Given a subgroup $H_{1}$ of a group $H$, we denote by $C\left(H_{1}\right)$ the centralizer of $H_{1}$ in $H$. Putting $\Gamma_{1}=C\left(\Gamma_{0}\right)$ and $U=C\left(\left(\Gamma_{0}\right)^{*}\right)(\mathrm{A} .10, \mathrm{~A} .12)$ we have $U=\Gamma_{1} \cdot \Gamma_{0}$ (cf. [22], proof of Lemma 6.5, pp. 502-503). Let us select a maximal abelian subgroup $H$ of $\Gamma_{1}$ and set $E=$ $\Gamma_{\mathbf{0}} \cdot H$.

LEMM 4 4.1.1. With the above notations we have (i) $E=C(H)$, (ii) $E$ is the direct product of $\Gamma_{0}$ and of a free abelian group $\Delta$.

Proof. Ad (i) We have evidently $E \subset C(H)$ and thus it is enough to establish the opposite inclusion.-If $\gamma \in C(H)$, since $H_{0}=\left(\Gamma_{0}\right)^{\star}$, we have $\gamma \in U$. Let us write 
$\gamma=\gamma_{1} \cdot \gamma_{0}\left(\gamma_{1} \in \Gamma_{1}, \gamma_{0} \in \Gamma_{0}\right)$. Since $H \subset \Gamma_{1}=C\left(\Gamma_{0}\right), \gamma_{1}$ commutes with $H$ and hence, since $H$ is maximal abelian in $\Gamma_{1}, \gamma_{1} \in H$ or $\gamma \in H \cdot \Gamma_{0}=E$.- Note that, in particular, $E$ is closed in $\Gamma$. Ad (ii) Since $H_{0}=H \cap \Gamma_{0}$, it suffices to show, that $H=H_{0} \times \Delta \cdot H$ being abelian, for this it is enough to establish, that $H /(H)_{0}$ is free abelian. We have

$$
H / H_{0}=H /\left(H \cap \Gamma_{0}\right)=H \Gamma_{0} / \Gamma_{0}=E / \Gamma_{0} \subset \Gamma / \Gamma_{0}
$$

whence the result follows by observing, that $\Gamma / \Gamma_{0}$ is free abelian.

Q.e.d.

CoRollary. $E$ is of type 1 .

In fact, by (ii) above it suffices to remark, that $\Gamma_{0}$ is a connected nilpotent Lie group.

In the following we shall associate with a given $E \in \hat{L} / \tilde{G}$ a closed subgroup $D$ of $G$, satisfying $K(E) \subset D \subset \mathfrak{U}(E) \subset G(E)$ (for $\mathfrak{U}(E)$ ef. III above). As the subsequent lemmas will show, $D$ shares all the useful properties of $K(E)$ and, in certain respects, it is more advantageous (cf. e.g. Lemma 4.1.3 and Remark 5.2 below). The reason for which, however, we prefer in our main statements $K(E)$ (cf. Proposition 1 and Theorem 2) is the lack of a possibility to make the choice of $D$ canonical.

Below we shall write $F, K, \mathfrak{U}$ and $G_{1}$ for $F(E), K(E), \mathfrak{U}(E)$ and $G(E)$ resp.-Let $\mathrm{T}$ be the central 1-torus in $G^{e}$. With the previous notations we set $D^{e}=\Phi^{-1}(E)$ and $D=$ $D^{e} / \mathbf{T} \subset G . D$ is a closed subgroup of $G$ such that $K \subset D \subset \mathfrak{U}$. To see this it is enough to recall, that $U^{*} \subset H \subset \Gamma_{1} \subset U$, and that $\mathfrak{U}=\Phi^{-1}(U) / \mathbf{T}$ (cf. [22], p. 483, bottom) and $K=$ $\Phi^{-1}\left(U^{*}\right) / \mathbf{T}$ (cf. loc. cit., proof of Lemma 3.6, p. 484).

LEMMA 4.1.2. Assume, that $\sigma$ is a factor representation of $D$ such that its restriction to $L$ is carried by E. Then $\sigma$ is of type $\mathrm{I}$.

Proof. Since $D \subset G_{1}$, the action of $D$ on $E$ is trivial, and thus $\sigma \mid L$ is quasi-equivalent to a $\pi^{\prime} \in E$. Replacing, if necessay, $\sigma$ by $a \sigma(a \in \tilde{G})$ we can assume, that $\pi^{\prime}=\pi$ ( $\pi$ as previously). Let us put $\Phi_{1}=\Phi \mid D^{e}$ and $\pi_{1}^{e}=\pi^{e} \mid D^{e}$. Denoting by $\mathcal{T}$ the l-torus in $\Gamma$, there is a $\mu \in$ Fac $(E)$ such that $\mu \mid \mathcal{J}$ is a multiple of the conjugate of the identity map of $\mathcal{T}$ onto itself, and that, with notations similar to those of Lemma 1.2.3, $\sigma=\left[\pi_{1}^{e} \otimes\left(\mu \circ \Phi_{1}\right)\right]^{\sim}$. Hence to complete our proof it is enough to recall, that $E$ is of type $I$ (cf. corollary of the previous lemma).

Q.e.d.

We set $F^{\prime}=\left\{\sigma ; \sigma \in \hat{D}, \sigma \mid L \approx \pi^{\prime}, \pi^{\prime}\right.$ any in $\left.E\right\}$

L Е м А 4.1.3. For any $\sigma$ in $F^{\prime}$, its stabilizer in $G$ is equal to $D$.

Proof. Since $[\tilde{G}, \tilde{G}]=L \subset D, D$ is an invariant subgroup of $\tilde{G}$. Replacing, if necessary, 8-742901 Acta mathematica 133. Imprimé le 7 Octobre 1974 
$\sigma$ by $b \sigma(b \in \tilde{G})$ we can assume from the beginning, that $\sigma \mid L \approx \pi$ (as previously), and then it is enough to show, that $a \sigma=\sigma$ (for some $a \in G$ ) implies $a \in D$.-If $a \sigma=\sigma$, then $a \pi=\pi$, or $a \in G_{1}$. In the following, whenever convenient, we shall write for $(g, 1),(e, u) \in G^{e}, g$ or $u$ resp. $\left(g \in G_{1}, u \in \mathbf{T}\right)$. There is a $\mu \in \operatorname{Irr}(E)$, such that $\mu \mid \mathbf{T}$ is a multiple of the conjugate of the identity map of $\mathbf{T}$ onto itself, and that $\sigma(d)=\pi^{e}(d) \otimes \mu(\Phi(d))(d \in D)$. Putting $\gamma=$ $\Phi((a, 1)) \in \Gamma$, we are going to show, that $\gamma \mu=\mu$. To this end it suffices to prove that, if we write $U=\pi^{e}(a) \otimes I$, we have $(a \sigma)(d) \equiv U^{*} \cdot\left(\pi^{e}(d) \otimes(\gamma \mu)(\Phi(d)) \cdot U(d \in D)\right.$. In fact, if again $\Phi_{1}=\Phi\left|D^{e}, \pi_{1}^{e}=\pi^{e}\right| D^{e}$, we obtain then

$$
\sigma=\left[\pi_{1}^{e} \otimes\left(\mu \circ \Phi_{1}\right)\right]^{\sim}, a \sigma=U^{*} \cdot\left[\pi_{1}^{e} \otimes\left((\gamma \cdot \mu) \circ \Phi_{1}\right)\right]^{\sim} \cdot U,
$$

whence, since $a \sigma \sim \sigma$, we conclude, that $\gamma \mu \sim \mu$ (A.5).--Let $d$ be fix in $D$. Then

$$
(a \sigma)(d)=\sigma\left(a^{-1} \cdot d a\right)=\pi^{e}\left(\left(a^{-1} \cdot d a, 1\right)\right) \otimes \mu\left(\Phi\left(\left(a^{-1} d a, 1\right)\right) .\right.
$$

Since $\left(a^{-1} d a, 1\right)=(a, 1)^{-1} \cdot(d, 1)(a, 1) \cdot u(u \in \mathbf{T})$, we obtain, that

$$
\begin{aligned}
(a \sigma)(d) & =\pi^{e}\left((a, 1)^{-1} \cdot(d, 1)(a, 1)\right) \otimes \mu\left(\Phi\left((a, 1)^{-1} \cdot(d, 1)(a, 1)\right)\right. \\
& =U^{*} \cdot\left(\pi^{e}(d) \otimes(\gamma \mu)(\Phi(d))\right) \cdot U,
\end{aligned}
$$

where $U=\pi^{e}(a) \otimes I$, as claimed above.-We show next, that $\gamma \mu=\mu$ implies that $\gamma \in E$. In fact, by $E=C(H)$ (Lemma 4.1.1, (i)) there is a $\chi \in X(H)$ such that $\mu \mid H \approx \chi$. Since $\gamma \mu=\mu$ we have also $\gamma \chi=\chi$. For a fixed $h \in H$, let us write $\gamma^{-1} h \gamma=u h(u \in \mathbf{T})$; we have to prove, that $u=1$. But (identifying $\mathbf{T}$ to the circle group) we get: $\chi(h)=(\gamma \chi)(h)=\chi\left(\gamma^{-1} h \gamma\right)=\bar{u} \chi(h)$, giving the desired conclusion.- Summing up, we have shown, that $a \sigma=\sigma$, implies that $a \in G_{1}$, and if $\gamma=\Phi((a, 1))$, then $\gamma \in E$. Hence $(a, 1) \in \Phi^{-1}(E)=D^{e}$ and $a \in D$, completing the proof of our lemma.

Q.e.d.

LEMMA 4.1.4. With the above notations $F^{\prime}$, as a subspace of $\hat{D}$, is locally compact and Hausdorff.

Proof. At many points this will be similar to that of Lemma 1.1.5. We show first as at loc. cit. (a), (b), that $F^{\prime}$ is locally closed in $\hat{D}$. Putting $\mathscr{D}=C^{*}(D)$ we conclude as in (c), loc. cit., that there are closed, two-sided ideals $\mathfrak{D} \supset I_{1} \supset I_{2}$ such that, if $M=I_{1} / I_{2}$, we have $\hat{M}=F^{\prime}$.-We prove next, that $M$ is of type $\mathrm{I}$ or, what amounts to the same, that if $\sigma^{\prime} \in \mathrm{Fac}\left(I_{1}\right)$ satisfies $\operatorname{ker}\left(\sigma^{\prime}\right) \supset I_{2}$, then $\sigma^{\prime}$ is of type I. We recall (cf. [4], 2.10.4, p. 52), that there is a unique $\sigma \in \mathrm{Fac}(D)$ such that $\sigma \mid I_{1}=\sigma^{\prime}$ and in this case $R(\sigma)=R\left(\sigma^{\prime}\right)$. This being so it is enough to show, that $\sigma \mid L$ is carried by $E$. In fact then, by Lemma 4.1.2, $\sigma$, and hence also $\sigma^{\prime}$, is of type $\mathrm{I}$. Let $\sigma=\int_{X}^{\oplus} \sigma(x) d \mu(x)$ be a decomposition into a direct integral of irreducible representations over the standard measure space $(X, \mu)$. There is a 
subset $n$ of $X$ with $\mu(\eta)=0$, such that if $x \in \eta, \operatorname{ker}(\sigma(x)) \equiv \operatorname{ker}(\sigma)$ (cf. [3], p. 100), hence also $\operatorname{ker}(\sigma(x)) \supset I_{2}$. There is also an $n_{1} \subset X, \mu\left(n_{1}\right)=0$ such that $\sigma(x) \mid I_{1} \neq 0$ if $x \in X-\boldsymbol{n}_{1}$. In fact, otherwise there is a nonzero projection $P$ with $P \sigma\left(I_{1}\right)=P \sigma^{\prime}\left(I_{1}\right)=0$. Since $F^{\prime}=$ $\left\{\delta ; \delta \in \hat{D}, \operatorname{ker}(\delta) \supset I_{1}, \delta \mid I_{2} \neq 0\right\}$ we conclude, that up to a set of measure zero, $\sigma(x) \in F^{\prime \prime}$; in which case also $\sigma(x) \mid L \approx \pi^{\prime} \in E$. Thus, on account of Lemma 1.1.7, $\sigma \mid L$ is carried by $E$. - We write $\mathcal{I}^{\prime}$ for the annihilator of $L$ in $X(D)$, and form the direct product $N^{\prime}=\tilde{G} \times \mathcal{I}^{\prime} \cdot N^{\prime}$ acts as a topological transformation group on $\hat{D}$ (cf. Corollary of Lemma 1.1.2). We claim, that $F^{\prime}$ is an $N^{\prime}$ orbit. To this end it suffices to show, that if $\alpha, \beta \in F^{\prime}$ are such, that $\alpha|L \approx \beta| L$, then there is a $\varphi \in \mathcal{I}$ with $\beta=\varphi \alpha$ (A.8). Let us write $\hat{E}^{\prime}$ for the subset of $\hat{E}$, which on $\mathcal{J}$ reduces to the conjugate of the identity map of $\mathcal{J}$. Putting, for $\eta \in \hat{E}^{\prime}, f(\eta)=\left[\pi_{1}^{e} \otimes\left(\eta \circ \Phi_{1}\right)\right]^{\sim}$ we can find $\gamma, \delta \in E^{\prime}$ such that $\alpha=f(\gamma), \beta=f(\delta)$. Also, one sees at once, that if $\mathcal{F}^{\prime \prime}$ is the annihilator of $\mathcal{J}$ in $X(E)$, there is an isomorphism $\tau: \mathcal{F}^{\prime} \rightarrow \mathcal{I}^{\prime \prime}$, such that $\varphi f(\eta)=f(\tau(\varphi) \eta)\left(\varphi \in \mathcal{F}^{\prime}\right)$. In this fashion it is enough to establish that, by multiplication, $\mathcal{F}^{\prime \prime}$ acts transitively on $\hat{E}^{\prime}$, By Lemma 4.1.1., (ii) we have $E=\Gamma_{0} \times \Delta$, and $\Gamma_{0}$ is of the form $\Gamma_{0}^{\prime} \times A$, where $A$ is a vector group, and $\Gamma_{0}^{\prime}$ central extension by the circle group of a vector group and, if $\operatorname{dim}(\Gamma)>1$, $\operatorname{dim}\left[\Gamma_{0}^{\prime}, \Gamma_{0}^{\prime}\right]=1$. Hence to obtain the desired conclusion it is enough to recall, that if $\chi \in X\left(\Gamma_{0}^{\prime}\right)$ is identically one on the center of $\Gamma_{0}^{\prime}$, and $\pi \in \hat{\Gamma}$, then $\varphi \pi=\pi$.-Summing up, $F^{\prime}=\hat{M}$ is locally compact and almost Hausdorff (since $M$ is of type $\mathrm{I}$ ) and $N^{\prime}$ acts tran. sitively on $\boldsymbol{F}^{\prime}$. In this fashion we can complete the proof of our lemma by reasoning as in (e) of the proof of Lemma 1.1.5.

Q.e.d.

LEMMA 4.1.5. (i) There is an equivalence relation $\Sigma^{\prime}$ on $F^{\prime}$, such that $\varrho_{1}$ is equivalent to $\varrho_{2}$ if and only if $\varrho_{1} \in \overline{G \varrho_{2}}$. (ii) $F^{\prime} / \Sigma^{\prime}$ is countably separated.

Proof. One can proceed in the same fashion, as in the proof of Lemma 1.1.6, and therefore we omit the details.

4.2. We continue to keep fixed a $J \in \operatorname{Prim}(G)$, which is not of type I, and set $E=E(J)$. $D$ will correspond to $E$ in the following as in 4.1.-Observe, that now $D \neq G$.

LEMMA 4.2.1. Suppose, that the normal representation $V$ of $G$ is of kernel $J$. There is a unitary representation $W$ of $D$, such that $V$ is quasi-equivalent to $\operatorname{ind}_{D \uparrow G} W$.

Proof. (a) Since $F^{\prime}=\hat{M}$, and $M$ is of type $I$ (cf. the proof of Lemma 1.1.4 above), we can find a Borel cross section $\sigma: F^{\prime} \rightarrow \operatorname{Irr}(D)$. Proceeding as in (a), proof of Lemma 1.2.1, we conclude, that there is a Borel measure $\lambda$ on $F^{\prime}$, which is quasi-invariant and ergodic with respect to $G$, such that $V \mid D=\int_{F}^{\oplus} \cdot \sigma^{\prime}(x) \cdot d \lambda(x)$, where $\sigma^{\prime}(x) \approx \sigma(x)$. Imitating the reasoning of (a), proof of Lemma 1.2.5, we can assume the following situation: (1) $\lambda$ is carried by 
the $\Sigma^{\prime}$ orbit $\mathcal{A} \subset F^{\prime},(2) \mathcal{A}$ is the underlying space of a connected abelian Lie group; there is a continuous homomorphism $\varphi: G \rightarrow \mathcal{A}$, with $\operatorname{ker}(\varphi)=D$, such that $\mathcal{A}^{\prime}=\varphi(G)$ implies $\overline{\mathcal{A}^{\prime}}=\mathcal{A}$, and $a \sigma^{\prime}(x)=\sigma^{\prime}(\varphi(a) x)(a \in G, x \in \mathcal{A})$. In the following we write $a x$ for $\varphi(a) x$.

(b) We have, by (a), $V \mid D=\int_{A}^{\oplus} \sigma^{\prime}(x) \cdot d \lambda(x)$, and the direct integral is clearly central. For $\psi \in X(\mathcal{A})$, let us put $V(\psi)=\int_{A}^{\oplus} \psi(x) \cdot I_{x} \cdot d \lambda(x)$; then $V(\psi) \in(R(V \mid D))^{\neq} \subset R(V)$. We define $\psi^{\prime} \in X(G)$ by $\psi^{\prime}(g) \equiv \overline{\psi(\varphi(g))}(g \in G)$. This being so we observe, that $V(g) V(\psi) V\left(g^{-1}\right)=$ $\psi^{\prime}(g) V(\psi)$.

(c) Let us denote by $C$ the annihilator of $D$ in $X(G)$. Putting $\mathcal{C}^{\prime}=\left\{\psi^{\prime} ; \psi \in X(A)\right\}$ we claim, that $C^{\prime}$ is dense in $C$. In fact, otherwise there is a $g \in G-D$ such that $1 \equiv \psi^{\prime}(g) \equiv$ $\overline{\psi(\varphi(g))}(\psi \in X(A))$, whence $\varphi(g)=e$ and $g \in \operatorname{ker}(\varphi)=D$, giving a contradiction.

(d) We write again $\left(\mathcal{S}=C^{*}(G)\right.$, and consider the homomorphism of Lemma 1.1.2, of $X(G)$ into Aut (G). Next we remark, that $(V(\psi))^{*} \cdot V(a) V(\psi) \equiv V\left(\psi^{\prime} a\right)$ for any $\psi \in X(\mathcal{A})$ and $a \in(G)$. In fact, fixing $\psi \in X(\mathcal{A})$, it is enough to show this for $a=f$, where $f$ is integrable with respect to a right invariant Haar measure $d g$. By (b) above we have $(V(\psi))^{*} \cdot V(g) V(\psi)=$ $\psi^{\prime}(g) V(g)$, and thus

$$
(V(\psi))^{*} \cdot V(f) \cdot V(\psi)=\int_{G} f(g)(V(\psi))^{*} \cdot V(g) \cdot V(\psi) \cdot d g=\int_{G} \psi^{\prime}(g) f(g) V(g) \cdot d g=V\left(\psi^{\prime} f\right)
$$

proving our assertion.

(e) Let us put $\mathbf{M}=R(V)$. Since $V \in \mathbf{F a c n}(G)$, in particular, $\mathbf{M}$ is a semifinite factor; we denote by $\Phi$ a faithful, normal, semifinite trace on $\mathbf{M}^{+}$. We claim, that for any $\chi \in \mathcal{C}$, $\Phi(V(\chi a)) \equiv \Phi(V(a))\left(a \in \mathbb{S}^{+}\right)$. In fact, since by $(\mathrm{d}),(V(\psi))^{*} \cdot V(a) \cdot V(\psi)=V\left(\psi^{\prime} a\right)(\psi \in X(\mathcal{A})$, $a \in G$ ) and since $V(\psi) \in \mathbf{M}$ (cf. (b)), our statement is certainly valid if $\chi \in \mathcal{C}^{\prime}$. By $\overline{\mathbf{C}}^{\prime}=\mathbf{C}$ (cf. (c)) and by the normalcy of $\Phi$ this yields $\Phi(V(\chi a)) \leqslant \Phi(V(a))$ and thus also $\Phi(V(\chi a)) \equiv$ $\Phi(V(a))$ for all $\chi \in \mathcal{C}$ and $a \in\left(\mathfrak{S}^{+}\right.$.

(f) For the following cf. [5], Ch. I, $\S 6,2$, pp. 85-89. Let us put $\mathrm{m}=\{A ; A \in \mathbf{M}$, $\left.\Phi\left(A^{*} \cdot A\right)<+\infty\right\} \cdot \mathfrak{m}$ can be endowed with the structure of a unitary algebra such that, for $X \in \mathfrak{m},(X, X)=\Phi\left(X \cdot X^{*}\right)$. We denote the Hilbert space, which is its completion, by $\mathfrak{h}$. Let us put $\mathfrak{n}=\left\{a ; a \in \mathbb{S}, \Phi\left(V\left(a^{*} \cdot a\right)\right)<+\infty\right\} ; \mathfrak{n}$ is a two-sided ideal in $\left(\mathfrak{S}\right.$. Writing $\mathfrak{h}^{\prime}=\{V(a) ; a \in \mathfrak{n}\} \subset \mathfrak{h}$, we have $\overline{\mathfrak{h}^{\prime}}=\mathfrak{h}$. In fact, by $V(\mathscr{S}) \mathfrak{h}^{\prime} \subset \mathfrak{h}^{\prime}, \mathfrak{h}^{\prime} V(\mathfrak{G}) \subset \mathfrak{h}^{\prime}$, if $P$ is the projection on $\overline{\mathfrak{h}^{\prime}}$, since $\mathbf{M}$ is a factor, $P$ is equal to zero or one. But the assumption, that $V$ is normal, clearly implies $P \neq 0$.-Let $g \mapsto L(g)(g \in G)$ be the left regular representation of $G$ on (S); we have $V(L(g) v)=$ $V(g) V(v)\left(v \in(\mathfrak{S})\right.$ and thus $L(g) \mathfrak{n} \subset \mathfrak{n}$. Hence there is a unitary representation $V^{\prime}$ of $G$ on $\mathfrak{h}$, uniquely determined by $V^{\prime}(g)(T)=V(g) T\left(T \in \mathfrak{h}^{\prime}\right) ; V^{\prime}$ is quasi-equivalent to $V$.-Also by (e), there is a unitary representation $W$ of $\mathcal{C}$ on $\mathfrak{h}$ such that, if $\psi \in \mathcal{C}, W(\psi)(V(v))=V(\psi v)$ $(v \in \mathfrak{n})$. One verifies easily, that $V^{\prime}(g) W(\psi) V^{\prime}\left(g^{-1}\right)=\overline{\psi(g)} W(\psi)(g \in G, \psi \in \mathcal{C})$. 
(g) Using the previous observations we complete the proof of our lemma as follows. We put $\bar{G}=G / D$ and, if $g \in G$, we denote its image in $\bar{G}$ by $\bar{g}$. Since $\bar{G}$ is the dual of $\mathrm{C}$, there is a spectral measure $\mathcal{D}$ on $\vec{G}$ such that $W(\psi)=\int_{\bar{G}} \psi(h) \cdot d \mathcal{D}(h)(\psi \in \mathcal{C})$. By the last relation of (f) we conclude, that if $E$ is some Borel subset of $\bar{G}$, we have $V^{\prime}(g) \mathcal{D}(E) V^{\prime}\left(g^{-1}\right)=$ $\mathcal{D}(E \bar{g})$. In other words, $\left(V^{\prime}, \mathcal{D}\right)$ constitutes a system of imprimitivity based on $G / D$, and hence (cf. [19], Theorem 6.6, p. 291) there is a $W \in \operatorname{Rep}(D)$, such that $V^{\prime}=\operatorname{ind}_{D \uparrow G} W$. Since (cf. (f)) $V \approx V^{\prime}, W$ satisfies the condition of our lemma.

Q.e.d.

For the notion of a multiplicity free representation of. [4], 5.4.5, p. 108 and 13.14, p. 250.-We shall say, that $W \in \operatorname{Rep}(D)$ is $G$ invariant, if $a W \sim W$ (A.5) for all $a \in G$.

Lемм 4 4.2.2. (i) $W$, as in Lemma 4.2.1, can be chosen $G$ invariant and multiplicity free, (ii) If $W$ and $W^{\prime}$ satisfy the conditions of (i) and $\operatorname{ind}_{D \uparrow G} W \approx \operatorname{ind}_{D \uparrow G} W^{\prime}$, then $W \sim W^{\prime}$.

Proof. Ad (i) Let $W$ be as in Lemma 4.2.1. Putting $W_{1}=\int_{G}^{\oplus} g W \cdot d g$, we have $a W_{1} \sim$ $W_{1}(a \in G)$ and ind ${ }_{D \uparrow G} W=(+\infty)\left(\operatorname{ind}_{D \uparrow G} W\right)$. Thus replacing $W$ by $W_{1}$, we can assume, that $W$ is $G$ invariant.-We claim, that in this case there is a $G$ invariant, multiplicity free representation $U$ of $D$ such that $W$ is a multiple of $U$. In fact, putting ind $d_{D \uparrow G} W=T \in \operatorname{Fac}_{(G)}$ we get, as in (a) of the proof of Lemma 4.2.1, since $G \neq D$,

$$
(+\infty) W=T \mid D=\int_{A}^{\oplus} \sigma^{\prime}(x) \cdot d \lambda(x)
$$

where $\lambda$ is a $G$ quasi-invariant and ergodic. On the other hand, since $F^{\prime}=\hat{M}$, and $M$ is of type I (cf. the proof of Lemma 4.1.4) there is a Borel measure $\mu$ on $F^{\prime}$, and a $\mu$-measurable function $x \mapsto n(x) \quad(x \in \mathcal{A})$ taking its values in the set of positive integers $(\leqslant+\infty)$ such that $W=\int_{F \cdot}^{\oplus} n(x) \cdot \sigma(x) \cdot d \mu(x)$ (cf. e.g. [4], 8.6.6. Théorème, p. 156); hence $\lambda$ is carried by $\mathcal{A}$, and there we can assume $\lambda=\mu$. Since $W$ is $G$ invariant and $\lambda$ ergodic, there is a positive integer $N(\leqslant+\infty)$ such that $n(x) \equiv N$ almost everywhere with respect to $\lambda$. We put $U=$ $\int_{A}^{\oplus} \sigma(x) \cdot d \lambda(x)$. Clearly $U$ is $G$ invariant, multiplicity free and $W=N \cdot U$. - In this fashion we can satisfy condition (i) of our lemma by replacing $W$ through $U$.-Ad (ii) Assume now, that $W$ and $W^{\prime}$ satisfy the conditions in (i) and that $\operatorname{ind}_{D \uparrow G} W \approx \operatorname{ind}_{D \uparrow G} W^{\prime}$. Then we have

$$
(+\infty) W=(\underset{D \uparrow G}{\operatorname{ind}} W)\left|D \approx\left(\underset{D \uparrow G}{\operatorname{ind}} W^{\prime}\right)\right| D=(+\infty) W^{\prime}
$$

and thus $(+\infty) W \sim(+\infty) W^{\prime}$. Since $W$ and $W^{\prime}$ are multiplicity free, we can conclude from here, that $W \sim W^{\prime}([4]$, 5.4.6. Proposition, p. 108).

Q.e.d.

We write $\tilde{N}$ for the direct product $\tilde{G} \times X(G)$ and consider again $N^{\prime}=\widetilde{G} \times \mathcal{F}^{\prime}$ as in the 
proof of Lemma 4.1.4. Putting, for $\chi \in X(G), \tau(\chi)=\chi \mid D$, and for $(a, \chi) \in \tilde{N}, j(a, \chi)=$ $(a, \tau(\chi)) \in N^{\prime}, j$ is a surjective homomorphism. In this fashion $\tilde{N}$ acts on $\hat{D}$, and $F^{\prime}$ is an $\tilde{N}$ orbit (cf. loc. cit.). Since $[\tilde{N}, \tilde{N}]=[\tilde{G}, \tilde{G}]=L \subset D \subset \tilde{N}_{\sigma}\left(\sigma \in F^{\prime}\right)$ we have $\tilde{N}_{\sigma} \equiv \Lambda$ (say), and $A=\tilde{N} / \Lambda$ is abelian. Let $\sigma$ be now fixed in $F^{\prime}$. We observe, that the map $a \Lambda \mapsto a \sigma(a \in \tilde{N})$ gives rise to an $\tilde{N}$ equivariant homeomorphism between $A$ and $F^{\prime}$ (cf. loc. cit. and (e), Lemma 1.1.5).--Let $\mathcal{A}$ be as in (a), Lemma 4.2.1, and assume that $\sigma$, as above, is in $\mathcal{A}$. Putting $\mathcal{E}=\{\alpha ; \alpha \in \tilde{N}, \alpha \mathcal{A} \subseteq \mathcal{A}\}$, we have also $\mathcal{E}=\{\alpha ; \alpha \in \tilde{N}, \alpha \sigma \in \mathcal{A}\}$. -We denote by $\hat{G}_{\text {norm }}$ the image of Facn $(\hat{G})$ in $\hat{G}$ (for the latter cf. [4], 18.6.2, p. 323). Setting again, if $V \in \operatorname{Rep}(G)$ and $\alpha=(b, \chi) \in \tilde{N},(\alpha V)=\chi(b V)$, since $\left(\alpha^{\prime} V\right)(v) \equiv V\left(\alpha^{-1}(v)\right) \quad\left(\alpha^{\prime}=(b, \bar{\chi}), v \in(S)\right.$, we get corresponding actions of $\tilde{N}$ on $\hat{G}_{\text {norm }}$ and Prim $(G)$. - Similarly, we can let $\tilde{N}$ act on $\operatorname{Rep}(D)$.

LEMMA 4.2.3. With the previous notations, we denote by $m$ the set of all $G$ quasi-orbits on $\mathcal{A}$, and write $G_{J}=\left\{l ; l \in \hat{G}_{\text {nurm }}\right.$, $\left.\operatorname{ker}(l)=J\right\}$. Then (i) $\mathcal{E}$ acts on $m$ and $\hat{G}_{J}$, (ii) There is an $\mathcal{E}$ equivariant injection $\eta: \hat{G}_{J} \rightarrow m$.

Proof. Ad (i) Given a Radon measure $\mu$ on $\mathcal{A}$ and $\alpha \in \mathcal{E}$, we define $\alpha \mu$ by $\int_{A} f(\alpha x) \cdot d \mu(x)=$ $\int_{A} f(x) \cdot d(\alpha \mu)(x)(f \in \mathcal{L}(\mathcal{A}))$. Since $G \subset \mathcal{E}$, and $\mathcal{E}$ acts on $\mathcal{A}$ as an abelian group, clearly $\mathcal{E}$ maps $m$ into itself.-We show next, that $\mathcal{E}$ leaves $\hat{G}_{J}$ invariant. To this end it is enough to establish, that if $V \in \operatorname{Facn}(G)$ is such, that $\operatorname{ker}(V)=J$, and if $\alpha \in \mathcal{E}$, then $\operatorname{ker}(\alpha V)=\operatorname{ker}(V)$. By Lemma 4.2.1, we can assume $V=\operatorname{ind}_{D \uparrow G} W$, whence $\alpha V=\operatorname{ind}_{D \uparrow G}(\alpha W)$. By virtue of Lemma 1.1.10, (i), it suffices to prove that ker $(\alpha W)=\operatorname{ker}(W)$. Let $M$ be as in the proof of Lemma 4.1.4, and let us denote by $T, T^{\prime}$ the representations of $M$ corresponding to $W$ and $\alpha W$ resp. Assuming, as we can by the previous lemma, that $W$ is $G$ invariant, the canonical measure $\lambda$ of $T$ on $\hat{M}=F^{\prime}$ is $G$ ergodic and is carried by $A \subset F^{\prime}$ where $A \in F^{\prime} / \Sigma^{\prime}$ depends on $J$ only (cf. [4], 8.6.8, p. 157). Hence the closed hull of $\lambda$ is equal to $\mathcal{A}$. The canonical measure of $T^{\prime}$ is just the action of $\alpha$ on $\lambda$; since $\alpha$ leaves $\mathcal{A}$ invariant the closed hull of this measure, too, coincides with $\mathcal{A}$. In this fashion $\operatorname{ker}(T)=\operatorname{ker}\left(T^{\prime}\right)$ (cf. loc. cit.) and hence also $\operatorname{ker}(W)=\operatorname{ker}(\alpha W)$, proving our statement.-Ad (ii) Let $l$ be some element of $\hat{G}_{\mathrm{J}}$. By the previous lemma, there is a $G$ invariant and multiplicity free $W \in \operatorname{Rep}(D)$, up to unitary equivalence uniquely determined by the condition, that the quasi-equivalence class of $\operatorname{ind}_{D \uparrow G} W$ be $l$. The canonical measure class of the representation, corresponding to $W$, of $M$ is a $G$ quasi-orbit on $\mathcal{A} \subset \hat{M}=F^{\prime}$ and is well determined by $l$; we write for it $\eta(l)(\in M)$. The map $l \mapsto \eta(l)$ from $\hat{G}_{J}$ into $m$ is clearly injective and, by what we saw in Ad (i) above, it is $\mathcal{E}$ equivariant.

Q.e.d.

4.2.4. For any element of $\hat{G}_{\text {norm }}$, its stabilizer in $\tilde{N}$ is closed.

Proof. We shall base this on the following two results, the first one of which is clas- 
sical. (1) Let $E$ be a countably separated Borel space, $G$ a separable locally compact group, acting on $E$ such that, for each fixed $x \in E$, the map $a \mapsto a x(a \in G)$ is Borel. Then $G_{x}$ (A.9) is closed. (2) $\hat{G}_{\text {norm }}$, as a subspace of the Borel space $\hat{G}$ is standard and thus, in particular, is countably separated. (This is implied by a recent result due to H. Halpern; cf. [15], Theorem 3.)-This being so to complete our proof it is enough to show, that for any $l$ fix in $\hat{G}_{\text {norm }}$, the map $\alpha \mapsto \alpha l(\alpha \in \tilde{N})$ is Borel. Let $V$ be some element of Fac (G) (cf. [14], p. 135), and $p$ the canonical projection from Fac $(G)$ onto $\hat{G}$. The map $\alpha \mapsto \alpha V$ is evidently Borel, hence so is $\alpha \mapsto \alpha l=p(\alpha V)(\alpha \in \tilde{N})$.

Q.e.d.

Proposition 4. Let $G$ be a connected and simply connected Lie group, and $V_{1}, V_{2}$ normal representations of $G$, such that $\operatorname{ker}\left(V_{1}\right)=\operatorname{ker}\left(V_{2}\right)$. Then $V_{1}$ is quasi-equivalent to $V_{2}$.

Proof. We assume, that $J$ and $\mathcal{A} \subset F^{\prime}$ are as before, and that $\operatorname{ker}\left(V_{j}\right)=J(j=1,2)$. -We start by observing, that there is a unique element $m \in m$ such that $\mathcal{E}_{m}=\mathcal{E}$ (A.9). In fact, with the notations of the remarks preceeding Lemma 4.2.3, putting $\Gamma=\mathcal{E} / \Lambda$ $(\subset A=\tilde{N} / \Lambda$ ), there is a $\Gamma$ equivariant homeomorphism between $\Gamma$ and $\mathcal{A}$. In this fashion we can derive our claim from the fact that, on $\Gamma$, any Borel measure, quasi-invariant with respect to $\Gamma$, is equivalent to the Haar measure (cf. [18], Lemma 7.3, p. 145).-With our previous notations our proposition states, that $\hat{G}_{J}$ contains only one element. Let $l \in \hat{G}_{J}$ and $\mu=\eta(l)$; by Lemma 4.2.3, (ii) it is enough to show, that $\mu=m$. By our previous remark, this will follow from $\mathcal{E}_{\mu}=\mathcal{E}$. By Lemma 4.2.4, $\mathcal{E}_{1}=\mathcal{E} \cap \tilde{N}_{1}$ is closed in $\mathcal{E}$. The $\mathcal{E}$ equivariance of $\eta$ implies $\mathcal{E}_{\mu}=\mathcal{E}_{1}$. On the other hand, evidently $G \subset \mathcal{E}_{\mu}, \Lambda \subset \mathcal{E}_{\mu}$ and $\overline{G \Lambda}=\mathcal{E}$. Thus finally $\overline{G \Lambda} \subseteq \mathcal{E}_{\mu} \subseteq \mathcal{E}$, providing $\mathcal{E}_{\mu}=\mathcal{E}$. $\quad$ Q.e.d.

Remark 4.2.1. Observe, that by what we have just seen, if $\lambda$ is an (evidently essentially uniquely determined) $G$ invariant Radon measure on $\mathcal{A}$, then $\int_{A}^{\oplus} \sigma(x) \cdot d \lambda(x) \in \operatorname{Rep}(D)$ induces in $G$ a normal representation of kernel $J$.

THEовем 1. Let $G$ be a connected Lie group. Then the map $l \mapsto \operatorname{ker}(l)$ from the set of all quasi-equivalence classes of normal representations into the space of primitive ideals is a bijection.

Proof. We start by recalling, that if $G$ is a locally compact group, $H$ a closed, invariant subgroup of $G, \Phi$ the canonical homomorphism from $G$ onto $G / H, V_{j} \in \operatorname{Rep}(G / H), V_{j}^{\prime}=$ $V_{j} \circ \Phi$, then $\operatorname{ker}\left(V_{1}\right)=\operatorname{ker}\left(V_{2}\right)$ is equivalent to $\operatorname{ker}\left(V_{1}^{\prime}\right)=\operatorname{ker}\left(V_{2}^{\prime}\right)$ - - Let now $G$ be as in our theorem, $G^{\prime}$ the universal covering of $G, \Phi$ the canonical homomorphism from $G^{\prime}$ onto $G$ and $\Gamma=\operatorname{ker}(\Phi)$. Given $V \in \operatorname{Rep}(G)$ we shall write $V^{\prime}=V \circ \Phi \in \operatorname{Rep}\left(G^{\prime}\right)$. We conclude from our starting remark, that given $J \in \operatorname{Prim}(G)$, there is a unique $J^{\prime} \in \operatorname{Prim}\left(G^{\prime}\right)$ such that, if $T \in \operatorname{Rep}(G), \operatorname{ker}(T)=J$, then $\operatorname{ker}\left(T^{\prime}\right)=J^{\prime}$, and conversely, if $U \in \operatorname{Rep}(G$ 
satisfies $\operatorname{ker}(U)=J^{\prime}$, then we have $U=V^{\prime}(V \in \operatorname{Rep}(G))$ with $\operatorname{ker}(V)=J$.- Let $d g$ and $d g^{\prime}$ be right invariant Haar measures on $G$ and $G^{\prime}$ resp., such that, if $d \gamma$ is the normalized invariant measure on $\Gamma$, we have $d g^{\prime}=d \gamma \cdot d g$. Let us put $\mathscr{G}=C^{*}(G)$ and $\mathfrak{S}^{\prime}=C^{*}\left(G^{\prime}\right)$. This being said we claim, that for any $T \in \operatorname{Rep}(G)$ we have $T(\mathcal{S})=T^{\prime}\left(\mathcal{S}^{\prime}\right)$. In fact, given $f \in \mathcal{L}\left(G^{\prime}\right)$, let us denote by $\tau(f)$ the element of $\mathcal{L}(G)$, corresponding to $x \mapsto \Sigma_{\gamma \in \Gamma} f(\gamma x)\left(x \in G^{\prime}\right)$; we have $T(\tau(f))=T^{\prime}(f)$. Since $\tau\left(\mathcal{L}\left(G^{\prime}\right)\right)=\mathcal{L}(G)$ this implies $T(\mathcal{L}(G))=T^{\prime}\left(\mathcal{L}\left(G^{\prime}\right)\right)$, and thus finally, since $T(\mathfrak{G}), T^{\prime}\left(\mathfrak{G}^{\prime}\right)$ are norm closed

$$
T(\mathbb{S})=\overline{T(\mathcal{L}(G))}=\overline{T^{\prime}\left(\mathcal{L}\left(G^{\prime}\right)\right)}=T^{\prime}\left(\mathcal{S}^{\prime}\right),
$$

proving our assertion. We conclude from this, that we have $V \in$ Facn $(G)$ if and only if $V^{\prime} \in \operatorname{Facn}\left(G^{\prime}\right)$. In fact to this end it is enough to recall (cf. [4], 6.7.2. Proposition, p. 127), that, for any locally compact group $G, V \in \mathrm{Facn}(G)$ is equivalent to the existence of a nonzero operator $A \in V(G)$ such that $A^{*} \cdot A$ is of finite trace.-Let now $J$ be an element of Prim $(G)$. Since $G^{\prime}$ is simply connected, by Proposition 2 there is a $W \in$ Facn $\left(G^{\prime}\right)$ with $\operatorname{ker}(W)=J^{\prime}$. What we said above implies, that we can find $V \in$ Facn $(G)$, such that $W=V^{\prime}$, and thus $\operatorname{ker}(V)=J$. Hence the map $l \mapsto \operatorname{ker}(l)$ from $\hat{G}_{\text {norm }}$ into Prim $(G)$ is surjective.If $V_{j} \in$ Facn $(G)$ and $\operatorname{ker}\left(V_{1}\right)=\operatorname{ker}\left(V_{2}\right)=J$, then $\operatorname{ker}\left(V_{1}^{\prime}\right)=\operatorname{ker}\left(V_{2}^{\prime}\right)$ and hence, by Proposition $4, V_{1}^{\prime} \approx V_{2}^{\prime}$. But then also $V_{1} \approx V_{2}$, completing the proof of our theorem. Q.e.d.

The following corollary has been known so far only if either $G$ is unimodular (implied by [4], 18.7.9, Corollaire, p. 326) or if $G$ is solvable ([22], Corollary 7.2, p. 594).

COROLLARY. Let $G$ be a connected Lie group. If $b$ is different from the unity, there is a normal representation of $G$, which on $b$ assumes a value different from the unit operator.

In fact, by the theorem of Gelfand and Raikov, there is a $U \in \operatorname{Irr}(G)$ such that $U(b) \neq I$. By Theorem 1 we can find a $T \in$ Facn $(G)$ with $\operatorname{ker}(T)=\operatorname{ker}(U)$. In this case, however, the kernels of these representations, viewed as homomorphisms of $G$ into unitary groups, too, must coincide, and hence $T(b)$ is different from the unit operator.

Remark 4.2.2. We wish to note here the following implication of the above results. Let us assume again, that $G$ is connected and simply connected, and let $J$ be some element of Prim $(G)$. By Proposition 2, there is a $T \in$ Facn $(G)$ with $\operatorname{ker}(T)=J$, and such that for some $\varphi \in B \cap\left(\mathfrak{S}^{+}=\mathcal{B}^{+}\right.$(cf. 2.1) $T(\varphi)$ is nonzero and of a finite trace. If $U \approx T, U(\varphi)$ is an operator of the same kind. But, by Proposition 4, $T$ is determined up to quasi-equivalence by its kernel. Hence we can conclude, that for any $V \in \operatorname{Facn}(G)$ there is a $\varphi \in \mathcal{B}^{+}$, such that $V(\varphi)$ is nonzero and of a finite trace.-Let $\Phi$ be a faithful, normal, semifinite trace on $R(V)$. Then the map $v \mapsto \Phi(V(v))(\leqslant+\infty)\left(v \in\left(\mathcal{S}^{+}\right)\right.$, up to a positive multiplicative 
constant, is uniquely determined by $J=\operatorname{ker}(V)$. We shall denote it by $\chi_{J}$, and call the character belonging to $J \in \operatorname{Prim}(G)$ (cf. [4], pp. 126-7). Let us observe, that by Remark 2.3.1 and by what we said above, if $\chi_{J_{1}}(v) \equiv \chi_{J_{2}}(v)\left(v \in \mathbf{B}^{+} ; J_{1}, J_{2}\right.$ fix in $\left.\operatorname{Prim}(G)\right)$, then $J_{1}=J_{2}$ - We recall finally, that if $G$ is solvable, by [22], Theorem 4 (p. 593), the Plancherel measure is carried by $\hat{G}_{\text {norm }}$, and there is a set $E \subset \hat{G}_{\text {norm }}$ of Plancherel measure zero, such that for any $V \in$ Facn $(G)$, the class of which belongs to $\hat{G}_{\text {norm }}-E$, there is a continuous positive definite function $\varphi$ of compact support, such that $V(\varphi)$ is nonzero and of a finite trace.

4.3. We recall (cf. Proposition 3), that $V \in$ Facn $(G)$ is of type $I$ if and only if, putting $J=\operatorname{ker}(V), \mathcal{A}(J)$ is $G$ transitive and $n(J)<+\infty$. The following proposition characterizes the situation arising upon dropping the second assumption on $J$.

Proposition 5. Let $G$ be a connected and simply connected Lie group. The following conditions bearing on the element $J$ of Prim $(G)$ are equivalent $(1) \mathcal{A}(J)$ is transitive with respect to $G$, (2) For any $\varrho \in \mathcal{A}(J)$, ind ${ }_{K(J) \uparrow G} \varrho$ is a normal representation.

Proof. $(1) \Rightarrow(2)$ We write $K=K(J)$.-Let us put, as before, $T(\varrho)=\operatorname{ind}_{K \uparrow G} \varrho(\varrho \in \mathcal{A}(J))$. If $\mu$ is a $G$ invariant Radon measure on $\mathcal{A}(J)$, by Lemma 2.3.6: $T=\int_{A(J)}^{\oplus} T(\varrho) \cdot d \mu(\varrho) \in$ Facn $(G)$. If $G$ acts transitively on $\mathcal{A}(J)$ we have, for any $\varrho \in \mathcal{A}(J), T(\varrho) \approx T$, and thus ind $\operatorname{lo}_{\uparrow} G \varrho$ is a normal representation.-(2) $\Rightarrow(1)$ We start by observing, that if $T(\varrho) \in$ Facn $(G)$ for one $\varrho \in \mathcal{A}(J)$, then the same holds true for all $\varrho$ in $\mathcal{A}(J)$. In fact, in this case even $T(\sigma) \in$ Facn $(G)$ for all $\sigma \in F(J)$ (cf. Notation 1.1.3). To see this we form again the direct product $\tilde{N}=$ $\tilde{G} \times X(G)$, and let it act, similarly as in Lemma 4.2.3, on $\operatorname{Rep}(G)$ and $\operatorname{Rep}(K)$. To obtain the desired conclusion it suffices to note, that $F(J)$ is an $\tilde{N}$ orbit (cf. (e), Lemma 1.1.5) and that $\alpha T(\varrho)=T(\alpha \varrho)(\varrho \in \mathfrak{U})$.-We are going to prove now, that $T(\varrho) \in$ Facn $(G)$ (for a single $\varrho \in \mathcal{A}(J))$ implies, that $G$ acts transitively on $\mathcal{A}(J)$, in the following fashion. Assume, that $\varrho_{1}, \varrho_{2} \in \mathcal{A}(J)$. By what we said above, we have then $T\left(\varrho_{1}\right), T\left(\varrho_{2}\right) \in$ Facn $(G)$ and also $\operatorname{ker}\left(T\left(\varrho_{1}\right)\right)=J\left(\varrho_{1}\right)=J=J\left(\varrho_{2}\right)=\operatorname{ker}\left(T\left(\varrho_{2}\right)\right)$. Hence, by Proposition 4, $T\left(\varrho_{1}\right) \approx T\left(\varrho_{2}\right)$, which, however, implies that $G \varrho_{1}=G \varrho_{2}$ (cf. Lemma 1.1.4, (ii)), or that $\mathcal{A}(J)$ is a $G$ orbit.

Q.e.d.

\section{$\$ 5$}

Let $G$ be again a connected and simply connected Lie group. If every normal representation of $G$ is of type I, by Proposition 2 and Lemma 3.1, $G$ itself must be of type I. Hence in order to form the character $\chi_{J}$ belonging to $J \in \operatorname{Prim}(G)$ (cf. Remark 4.2.2) we have to consider traces on factors which, in general, are not of type $I$. The purpose of this section is to find for $\chi_{J} \mid \boldsymbol{B}^{+}$an expression in terms of type $I$ traces (cf. Theorem 2 below). -We recall (cf. loc. cit.) that $\chi_{J_{1}}\left|B^{+}=\chi_{J_{2}}\right| \mathcal{B}^{+}$implies $J_{1}=J_{2}$. 8†-742901 Acta mathematica 133. Imprimé le 7 Octobre 1974 
LemMa 5.1. Assume, that $J$ and $D$ are as in 4.2. There is a closed subgroup $S$ of $G$, such that $D \subset S \subset G$ and $S / D$ is discrete, and a unitary representation $W$ of $D$ with the following properties (i) Putting $V=\operatorname{ind}_{D \uparrow G} W$, we have $V \in$ Facn $(G)$ and $\operatorname{ker}(V)=J$. (ii) $W$ is multiplicity free and $s W \sim W(s \in S)$, (iii) Writing $U=\operatorname{ind}_{D \uparrow s} W, R(V)$ is tensor product of $R(U)$ with the full ring of a Hilbert space.

Proof. (a) Let $\mathcal{A}$ be as in the proof of Proposition 4. By choosing an origin for the action of $\Gamma$ as loc. cit. we can assume, that $\mathcal{A}$ itself is a connected abelian Lie group. As in Remark 4.2.1 we form the representation $Z=\int_{A}^{\oplus} \sigma(x) \cdot d \lambda(x)$; by what we said loc. cit. $\operatorname{ind}_{D \uparrow G} Z \in$ Facn $(G)$ is of kernel $J$, and clearly $\lambda$ is just the Haar measure on $\mathcal{A}$. We have a homomorphism $\varphi: G \rightarrow \mathcal{A}$ such that $\operatorname{ker}(\varphi)=D, \varphi(G)=\mathcal{A}^{\prime}$ implies $\overline{A^{\prime}}=\mathcal{A}$, and $a \sigma(x)=$ $\sigma(\varphi(a) x)(a \in G, x \in \mathcal{A})$; we write $a x$ in place of $\varphi(a) x$ in the sequel.-As in (b), proof of Lemma 1.2.5, we observe, that there is a closed, connected subgroup $\mathcal{A}_{1}$ of $\mathcal{A}$, such that $\mathcal{A}^{\prime} \cdot \mathcal{A}_{1}=\mathcal{A}$, and $\mathcal{A}_{1} \cap \mathcal{A}^{\prime}=\mathcal{A}_{1}^{\prime}$ is countable and dense in $\mathcal{A}_{1}$. Let us put $S=\varphi^{-1}\left(\mathcal{A}_{1}^{\prime}\right)$. $S$ is closed, contains $D, S / D$ is discrete and $\mathfrak{B}=\mathcal{A} / \mathcal{A}_{1}=G / S$. Let $\mu$ and $h$ be Haar measures on $A$ and $B$ resp. such that $d \lambda=d \mu \cdot d h$. For $p \in \mathfrak{B}$, we write $\mu_{p}$ for the translate of $\mu$ carried by the coset $p$; then $\lambda=\int_{B}^{\oplus} \mu_{p} \cdot d h(p)$. Putting

$$
T(p)=\int_{A}^{\oplus} \sigma(x) \cdot d \mu_{p}(x)
$$

we have (cf. [17], Theorem 2.11, p. 204)

$$
\int_{\mathfrak{B}}^{\oplus} T(p) \cdot d h(p)=\int_{A}^{\oplus} \sigma(x) \cdot d \mu(x)=Z .
$$

Since clearly $a T(p)=T(a p)(a \in G, p \in \mathfrak{B})$, setting $W=T(0)$, where 0 is now the neutral element of $\mathfrak{B}$, we conclude (cf. [16], Theorem 10.1, p. 123) that

$$
\operatorname{ind}_{D \uparrow G} Z=\int_{\mathfrak{B}}^{\oplus}\left(\operatorname{ind}_{D \uparrow G} T(p)\right) \cdot d \nu(p) \approx \operatorname{ind}_{D \uparrow G} W .
$$

Hence, putting $V=\operatorname{ind}_{D \uparrow G} W$, we get $V \in \operatorname{Facn}(G)$ and $\operatorname{ker}(V)=J$. Since $W$ is multiplicity free and clearly $s W \sim W(s \in S$ ), it satisfies conditions (i)-(iii) of our lemma. Hence it remains only to verify condition (iii).

(b) Putting $U=\operatorname{ind}_{D \uparrow S} W$, we obtain $V=\operatorname{ind}_{S \uparrow G} U$ and $V \mid S=\int_{G / S}^{\Theta_{S}} h U \cdot d h$.

(A.11). We claim, that the last decomposition is central. To this end it is enough to show the same for $\int_{G / S}^{\oplus} h(U \mid D) \cdot d h$. By $s W \sim W(s \in S)$ we have $U \mid D=\int_{S_{/ D}}^{\oplus_{D}} W \cdot d \sigma \sim W$; hence the desired conclusion follows by observing, that clearly $\int_{G / S}^{\oplus} h W \cdot d h(=Z)$ is central.

(c) The subsequent analysis, in view of applications in the next lemmas, is somewhat more detailed, than needed to complete the proof of our lemma. In order to realize $V=$ 
ind $_{S \uparrow G} U$, we construct a Borel cross section $\eta: \mathfrak{B} \rightarrow G$ and an open subset $O \subset \mathfrak{B}$, the complement of which is of Haar measure zero, such that $\eta \mid O$ be continuous, as follows. Since $\mathfrak{B}$ is a connected abelian Lie group, there are one-parameter subgroups $\left\{g_{k}(t) ; t \in \mathbf{R}, 1 \leqslant k \leqslant n\right\}$ $\left\{g_{1}^{\prime}(\tau) ; \tau \in \mathbf{T}, 1 \leqslant l \leqslant m\right\} \quad\left(\mathbf{T}=\right.$ circle group) of $\mathfrak{B}$, such that putting $t=\left(t_{1}, t_{2}, \ldots, t_{n}\right) \in \mathbf{R}^{n}, \tau=$ $\left(\tau_{1}, \tau_{2}, \ldots, \tau_{m}\right) \in \mathbf{T}^{m}$, the $\operatorname{map}(t, \tau) \mapsto g_{1}\left(t_{1}\right) g_{2}\left(t_{2}\right) \ldots g_{n}\left(t_{n}\right) g_{1}^{\prime}\left(\tau_{1}\right) g_{2}\left(\tau_{2}\right) \ldots g_{m}^{\prime}\left(\tau_{m}\right)$ is a homeomorphism. We denote by $\omega$ and $\varphi$ the canonical homomorphisms $G \rightarrow \mathfrak{B}$ and $\mathbf{R} \rightarrow \mathbf{T}$ resp. Let $\left\{e_{k}(t) ; t \in \mathbf{R}, 1 \leqslant k \leqslant n\right\},\left\{e_{l}^{\prime}(t) ; t \in \mathbf{R}, 1 \leqslant l \leqslant m\right\}$ be one-parameter subgroups of $G$, such that $\omega\left(e_{k}(t)\right) \equiv g_{k}(t) \quad(1 \leqslant k \leqslant n)$ and $\omega\left(e_{l}^{\prime}(t)\right) \equiv g_{l}^{\prime}(\varphi(t))(1 \leqslant l \leqslant m)$. We identify $\mathbf{T}$ to $[0,1]$ modulo 1 , and define $\psi: \mathbf{T} \rightarrow[0,1]$ by $\psi(\tau)=\tau$ if $0<\tau<1$, and $\psi(\tau)=0$ otherwise. Let $\eta: \mathfrak{B} \rightarrow G$ be such that

$$
\eta(t, \tau)=e_{1}\left(t_{1}\right) e_{2}\left(r_{2}\right) \ldots e_{n}\left(t_{n}\right) e_{1}^{\prime}\left(\psi\left(\tau_{1}\right)\right) e_{2}^{\prime}\left(\psi\left(\tau_{2}\right)\right) \ldots e_{m}^{\prime}\left(\psi\left(\tau_{m}\right)\right)
$$

Clearly, $\eta$ is Borel and $\omega \circ \eta=$ identity. We set

$$
\mathcal{O}=\left\{(t, \tau) ; 0<\tau_{k}<1, \quad 1 \leqslant k \leqslant m\right\}
$$

Then $O$ is open in $\mathfrak{B}$ such that $h(\mathfrak{B}-O)=0$, and $\eta \mid O$ is continuous. For later use observe, that $d h \mid \mathcal{O}=d t \cdot d \tau\left(d t=d t_{1} d t_{2} \ldots d t_{n}, d \tau=d \tau_{1} d \tau_{2} \ldots d \tau_{m}\right) .(2)$ Let us put $T=\eta(\mathfrak{B})$; then any $a \in G$ can be written uniquely as $s t(s \in S, t \in T)$. Given $a \in G$ and $h \in \mathfrak{B}$ we write $h \bar{a}=h \omega(a)$; hence if $t \in T, \eta(h) \cdot t=\gamma(h, t) \cdot \eta(h \bar{t})(\gamma(h, t) \in S)$. Observe, that if $s \in S, t \in T$, then

$$
\eta(h) s \cdot t=\left[\eta(h) \cdot s(\eta(h))^{-1} \cdot \gamma(h, t)\right] \eta(h \bar{t}) .
$$

For notational convenience, we shall write in the following sometimes $h$ in place of $\eta(h) \in T$ $(\subset G)$ and thus, in particular, hā for $\eta(h \omega(a))(a \in G)$. (3) Let us set $\mu(h, t) \equiv U(\gamma(h, t))(h \in \mathfrak{B}$, $t \in T)$. From what we have just seen we conclude, that $V=\operatorname{ind}_{S \uparrow G} U$ can be realized on $H(V)=H(U) \otimes L_{h}^{2}(\mathcal{B})$ by

$$
(V(s t) f)(h) \equiv\left(h^{-1} U\right)(s) \mu(h, t) f(h \bar{t}) \quad(w \in S, t \in T ; f \in H(V))
$$

(d) The following reasoning is inspired by [19], pp. 288-290.-By (b) the decomposition $V \mid S=\int_{\mathfrak{B}}^{\oplus}\left(h^{-1} U\right) \cdot d h$ is central. Therefore, if $A \in(R(V))^{\prime}$, we can write by virtue of it: $A=\int_{0} A(h) \cdot d h$, where $A(h) \in(R(U))^{\prime}$ almost everywhere. Hence if $t \in T$ is fixed and $f \in H(V)$, we have $(A V(t) f)(h) \equiv \mu(h, t) \cdot A(h) f(h \bar{t})$ on the one hand; on the other, this is identical to $(V(t) A f)(h) \equiv \mu(h, t) A(h \bar{t}) f(h \bar{t})$ for almost all $h \in \mathfrak{B}$ In this fashion $A(h \bar{t}) \equiv A(h)$ and, in view of the aribtrariness of $t \in T$, there is a $A \in(R(U))^{\prime}$ such that $A(h) \equiv A$. In other words $(R(V))^{\prime}=(R(U))^{\prime} \otimes I$ and hence $R(V) \stackrel{=}{=} R(U) \otimes B\left(L_{h}^{2}(\mathfrak{B})\right.$ ) (A.13) completing the proof of Lemma 5.1.

$$
\text { Q.e.d. }
$$

Let $d x, d s$ be right invariant Haar measures on $G$ and $S$ resp., such that $d x=d s \cdot d h$. 9-742901 Acta mathematica 133. Imprimé le 4 Octobre 1974 
We write $\mathbb{B}=C^{*}(G), \subseteq=C^{*}(S)$ and consider as in 2.1, the variety $\mathbb{B} \subset(\mathbb{S}$. We observe, that $B \mid S \subset B_{S} \subset \subseteq$ (cf. Remark 2.1.2) and that $B^{+} \mid S \subset B_{S}^{+} \subset \bigodot^{+}$.- Since $V \in$ Facn $(G), R(V)$ is a semifinite factor and hence, by (iii) of the above lemma, so is $R(U)$.

LEMMA 5.2. Assume, that $V$ and $U$ are as in Lemma 5.1. Let $\Psi$ be a faithful, normal, semifinite trace on $[R(U)]^{+}$. There is a trace $\Phi$ of the same kind on $[R(V)]^{+}$, uniquely determined by

$$
\Phi(V(\varphi))=\int_{G / S} \Psi((h U)(\varphi \mid S)) \cdot d h \quad\left(\varphi \in \mathcal{B}^{+}\right)
$$

Proof. Uniqueness is clear, since there is a $\varphi \in B^{+}$, such that $0<\Phi(V(\varphi))<+\infty$ (cf. Remark 4.2.2).-We recall (cf. (c. 2), proof of the previous lemma), that $h(\in \mathfrak{B})$ can stand also for $\eta(h) \in G$.

(a) Given $\varphi \in \mathbb{B}$ and $h, t$ fix in $\mathfrak{B}$, let us put $F(x) \equiv\left(h^{-1} U\right)\left(\varphi\left(h^{-1} x t\right)\right) U(x)(x \in S)$. One verifies at once, that $F(l x) \equiv \dot{F}(x) \quad(l \in L, x \in S)$. We set $\Sigma=S / L$, and denote by $d l$, $d \sigma$ Haar measures on $L$ and $\Sigma$ resp., such that $d s=d l \cdot d \sigma$ By what we have just said we can form $\int_{\Sigma}\left(h^{-1} U\right)\left(\varphi\left(h^{-1} x t\right)\right) U(x) \cdot d \sigma \equiv K_{\psi}(h, t)$ (say). We observe, that the map $(h, t) \mapsto$ $K_{\varphi}(h, t)$ is continuous from $O \times O$ into $B(H(U))$, the latter being taken in the uniform topology (cf. loc. cit. (c.1)).-We denote by $\vartheta$ the linear variety in $H(V)=H(U) \otimes L_{h}^{2}(B)$ composed of all those continuous maps from $\mathfrak{B}$ into $H(U)$, which are of a compact support contained in $O$. We are going to show that, for any $f \in V,(V(\varphi) f)(h)=\int_{\bigotimes} K_{\varphi}(h, t) f(t) \cdot d t$. In fact, let us suppose first that $\varphi \in \mathcal{B}_{0}(\sim \mathcal{L}(G)$; cf. Remark 2.1.1). Writing $d h$ also for $\eta_{*}(d h)$ on $T=\eta(\mathfrak{B})$, we have $\int_{G} \varphi(g) \cdot d g=\int_{s \times T} \varphi(s t) \cdot d s \cdot d t$. In this fashion, by (c.3) in the proof of Lemma 5.1, we obtain

$$
\begin{aligned}
(V(\varphi) f)(h) & =\int_{S \times T} \varphi(s t)(V(s t) f)(h) \cdot d s \cdot d t=\int_{S \times T} \varphi(s t)\left(h^{-1} U\right)(s) \mu(h, t) f(h t) \cdot d s \cdot d t \\
& =\int_{T}\left(\int_{S} \varphi(s t) U\left(h s h^{-1}\right) \cdot d s\right) \mu(h, t) f(h t) d t
\end{aligned}
$$

Writing $d\left(g_{0} \cdot g\right)=\Delta\left(g_{0}\right) d g$, since $[G, G]=L \subset S$, we have also $d\left(h s h^{-1}\right)=\Delta(h) d s(h \in G)$. Hence the substitution $s \rightarrow h^{-1} s h$ yields

$$
(V(\varphi) f)(h)=(\Delta(h))^{-1}\left(\int_{T}\left(\int_{S} \varphi\left(h^{-1} s h t\right) U(s) \cdot d s\right) \mu(h, t) f(h \bar{t}) \cdot d t\right.
$$

Recalling, that $h t=\gamma(h, t) \cdot h \bar{t}$ (loc. cit. (c. 2)) and $\mu(h, t) \equiv U(\gamma(h, t))$ (loc.cit (c.3)), we obtain through the further substitution $s \rightarrow s(\gamma(h, t))^{-1}$ 


$$
\begin{aligned}
(V(\varphi) f)(h) & =(\Delta(h))^{-1}\left(\int_{T}\left(\int_{S} \varphi\left(h^{-1} s h \bar{t}\right) U(s) \cdot d s\right) f(h \bar{t}) \cdot d t\right) \\
& =(\Delta(h))^{-1}\left(\int_{\mathfrak{B}}\left(\int_{S} \varphi\left(h^{-1} s t\right) U(s) \cdot d s\right) f(t) \cdot d t\right)=\int_{\mathfrak{B}} H(h, t) f(t) \cdot d_{t}
\end{aligned}
$$

where, noting that $d\left(h l h^{-1}\right)=\Delta(h) \cdot d l$, with notations as at the start of 2.1 , we have

$$
\begin{aligned}
H(h, t) & \equiv(\Delta(h))^{-1}\left(\int_{S} \varphi\left(h^{-1} s t\right) U(s) \cdot d s\right) \equiv(\Delta(h))^{-1}\left(\int_{\Sigma}\left(\int_{L} \varphi\left(h^{-1} l s t\right) U(l) \cdot d l\right) U(s)\right) \cdot d \sigma \\
& \left.\equiv \int_{\Sigma}\left(\int_{L} \varphi\left(l h^{-1} s t\right)\left(h^{-1} U\right)(l) \cdot d l\right) U(s)\right) d \sigma \equiv \int_{\Sigma}\left(h^{-1} U\right)\left(\mathcal{I}(\varphi)\left(h^{-1} s t\right)\right) U(s) \cdot d \sigma \equiv K_{p}(h, t) .
\end{aligned}
$$

If $\varphi \in B$ is arbitrary, we can find a sequence $\left\{\varphi_{n}\right\} \subset B_{0}$ such that $\operatorname{supp}\left(\varphi_{n}\right)$ is carried by a fixed compact subset $C$ of $G / L$ and $\varphi-\varphi_{n} \rightarrow 0$ uniformly (cf. loc. cit.). This implies, by what we saw above, at once

$$
(V(\varphi) f)(h) \equiv \int_{\mathfrak{B}} K_{\varphi}(h, t) f(t) \cdot d t \quad(f \in \mathcal{Y}),
$$

where $K_{p}(h, t) \equiv \int_{\Sigma}\left(h^{-1} U\right)\left(\varphi\left(h^{-1} s t\right)\right) U(s) \cdot d \sigma$, as claimed before.

Q.e.d.

(b) From now on we shall assume, that $\varphi \in B^{+}$. Let $e \in H(U)$ be fixed. We assert, that the function $(h, t) \mapsto\left(K_{\varphi}(h, t) e, e\right)$ is positive definite and continuous on $\mathcal{O} \times \mathcal{O}$. To see this, we select a $\lambda \in \mathcal{L}(O)(\mathrm{A} .7)$, set $f=e \otimes \lambda \in H(V)$ and observe, that

$$
(V(\varphi) f, f)=\int_{\mathfrak{B} \times \mathfrak{B}}\left(K_{\varphi}(h, t) e, e\right) \overline{\lambda(h)} \lambda(t) \cdot d h \cdot d t \geqslant 0,
$$

which implies the desired conclusion.--Note that, in particular, $K_{\varphi}(h, h) \geqslant 0(h \in O)$.

(c) We recall, that writing $B$ for the full ring of $L_{h}^{2}(\mathfrak{B})$, we have $R(V)=R(U) \otimes B$ (cf. Lemma 5.1, (iii)). Hence, since $V \in$ Facn $(G)$ along with $R(V), R(U)$ is a semifinite factor. Let $\Psi$ be a faithful, normal, semifinite trace on $R(U)$. We are going to show, that there is a trace $\Phi$ of the same kind on $R(V)$, such that

$$
\Phi(V(\varphi))=\int_{\mathfrak{B}} \Psi\left(K_{\varphi}(h, h)\right) \cdot d h \quad\left(\leqslant+\infty ; \varphi \in \mathcal{B}^{+}\right) .
$$

We start by recalling (cf. [5], Corollaire, p. 85), that there is a family $\left\{e_{j} ; j \in I\right\} \subset H(U)$ with $\Psi(A)=\Sigma_{j \in I}\left(A e_{j}, e_{j}\right)\left(A \in[R(U)]^{+}\right)$. We can assume $S \neq G$. Let $\left\{\lambda_{n} ; n=1,2, \ldots\right\} \subset \mathcal{L}(O)$ be a complete orthonormal system in $L_{h}^{2}(\mathfrak{B})$, and $\psi_{j, n}=e_{j} \otimes \psi_{n}$. We denote by $\Phi$ the faithful, normal, semifinite trace defined on $[R(V)]^{+}$by $\Phi(A)=\Sigma_{j, n}\left(A \psi_{j, n}, \psi_{j, n}\right)$ and show below, that it meets our condition. We write $I=(0,1)$, and recall (cf. (c. 1), Lemma 5.) that 
$\mathfrak{B} \supset O$ is homeomorphic to $R^{n} \times \mathbf{I}^{m}$ such that $d h \mid O=d t \cdot d \tau$. For $j \in I$ fixed, let us put $K_{j}(h, k) \equiv\left(K_{q}(h, k) e_{j}, e_{j}\right)$. Since (cf. (b) above) $(h, k) \mapsto K_{j}(h, k)$ is positive definite and continuous on $O \times O$, by the theorem of Mercer

$$
\sum_{n=1}^{\infty} \int_{o \times 0} K_{j}(h, k) \overline{\lambda_{n}(h)} \lambda_{n}(k) \cdot d h \cdot d k=\int_{0} K_{j}(h, h) \cdot d h \leqslant+\infty .
$$

Hence we conclude that

$$
\begin{aligned}
\Phi(V(\varphi)) & =\sum_{n, j}\left(V(\varphi) \psi_{j, n}, \psi_{j, n}\right)=\sum_{j}\left(\sum_{n=1}^{\infty} \int_{\mathfrak{B}} K_{f}(h, k) \overline{\lambda_{n}(h)} \lambda_{n}(k) \cdot d h \cdot d k\right) \\
& =\sum_{j} \int_{\mathfrak{B}} K_{j}(h, h) \cdot d h=\int_{\mathfrak{B}}\left(\sum_{j} K_{j}(h, h) \cdot d h\right)
\end{aligned}
$$

Since $K_{j}(h, h) \equiv\left(K_{\varphi}(h, h) e_{j}, e_{j}\right)$, we have $\sum_{j} K_{j}(h, h)=\Psi\left(K_{\varphi}(h, h)\right)$ whence $\Phi(V(\varphi))=$ $\int_{\mathfrak{B}} \Psi\left(K_{\varphi}(h, h)\right) \cdot d h \quad(\leqslant+\infty)$.

(d) We observe next, that if $\varphi \in \mathcal{B}$, then $\varphi \mid S \in B_{S} \subset \subseteq=C^{*}(S)$ and $K_{\varphi}(h, h)=\left(h^{-1} U\right)(\varphi \mid S)$. In fact, since (cf. (a))

$$
K_{\varphi}(h, t)=\int_{\Sigma}\left(h^{-1} U\right)\left(\varphi\left(h^{-1} s t\right)\right) U(s) \cdot d \sigma
$$

we have (cf. Remark 2.1.2)

$$
K_{\varphi}(h, h)=\int_{\Sigma}\left(h^{-1} U\right)\left(\varphi\left(h^{-1} s h\right)\right) U(s) \cdot d \sigma=\int_{\Sigma}\left(h^{-1} U\right)(\varphi(s))\left(h^{-1} U\right)(s) \cdot d \sigma=\left(h^{-1} U\right)(\varphi \mid S)
$$

Hence, by the last relation of (c) we obtain finally

$$
\Phi(V(\varphi))=\int_{\mathfrak{B}} \Psi((h U)(\varphi \mid S) \cdot d h
$$

completing the proof of Lemma 5.2.

Q.e.d.

Below we shall write $\operatorname{Tr}($ ) for the faithful, normal, semifinite trace, with the standard normalization, on the full ring of a Hilbert space.

Remark 5.1. We shall use later the following byproduct of the previous proof. Assume, that $M$ and $N$ are closed, but not necessarily connected subgroups of $G$ such that $M \supset N \supset L$. We suppose also, that . (ind $\left.{ }_{w \uparrow M} \alpha\right)=\beta \in \operatorname{Irr}(M)$, and that $\beta \mid N=\int_{M / N}^{\oplus_{N}} p \alpha \cdot d p$ (A.11) is central. Then for any $g \in \boldsymbol{B}_{M}^{+}$

$$
\operatorname{Tr}(\beta(g))=\int_{M / N} \operatorname{Tr}((p \alpha)(g \mid N)) \cdot d p \quad(\leqslant+\infty)
$$


We write $\chi_{J}(v)=\Phi(V(v))\left(v \in\left(S^{+} ; J=\operatorname{ker}(V)\right)\right.$ (cf. Remark 4.2.2). Below $D, \mathcal{A}$ and $\lambda$ are as in $(a)$, proof of Lemma 5.1.

Lemma 5.3. Assuming suitable normalization of $\lambda$, we have

$$
\chi_{J}(\varphi)=\int_{A} \operatorname{Tr}(\sigma(\varphi \mid D)) \cdot d \lambda(\sigma) \quad\left(\varphi \in \mathcal{B}^{+}\right) .
$$

Proof. (a) We write again $\mathfrak{D}=C^{*}(D)$ and $\subseteq=C^{*}(S)$. Since $S / D$ is discrete (cf. Lemma 5.1 , we can identify $\mathfrak{D}$ to a closed subalgebra of $S$.-Let us denote by $\mathcal{C}$ the annihilator of $D$ in $X(S)$. C is compact, and we write $d \chi$ for the normalized invariant measure on $C$. We consider, as in Lemma 1.1.2, the representation $\chi \mapsto A(\chi)$ of $\mathcal{C}$ in Aut ((S); we recall, that $T(A(\chi) v)=(\chi T)(v)(T \in \operatorname{Rep}(S), v \in \Subset, \chi \in C)$. Let us put $P=\int_{\mathrm{c}} A(\chi) \cdot d_{\chi} . P: \subseteq \rightarrow \mathfrak{D}$ is continuous, such that $P \mid \mathfrak{D}$ is the identity map; moreover $P\left(S^{+}\right)=\mathfrak{D}^{+}$and, if $\varphi \in \mathfrak{B}_{S}$, $P \varphi=\varphi \mid D \in B_{D}$.

(b) Assuming, that $U$ and $\Psi^{*}$ are as in Lemma 5.2, we show next, that $\Psi(U(v)) \equiv$ $\Psi^{\prime}(U(P v))\left(v \in \widetilde{S}^{+}\right)$. In fact, since $U=\operatorname{ind}_{D \uparrow S} W$, we have $\chi U \sim U(\chi \in \mathcal{C})$, hence

$$
\Psi\left(U(A(\chi) v) \equiv c(\chi) \Psi(U(v)) \quad\left(v \in \Im^{+}\right) .\right.
$$

Obviously $c\left(\chi_{1}\right) c\left(\chi_{2}\right)=c\left(\chi_{1} \cdot \chi_{2}\right)$ and $c(\chi)>0$ on $C$ and thus, by the compactness of $\mathcal{C}, c(\chi) \equiv 1$ and therefore also $\Psi\left(U(A(\chi) v) \equiv \Psi(U(v))\left(v \in \Im^{+}, \chi \in \mathcal{C}\right)\right.$. Fixing $v \in \mathfrak{S}^{+}$, if $e$ is arbitrary in $H(U)$, we have $(U(A(\chi) v) e, e) \geqslant 0$. Hence (cf. (c), Lemma 5.2)

$\Psi(U(v))=\int_{C} \Psi(U(A(\chi) v)) \cdot d \chi=\sum_{j \in I} \int_{C}\left(U(A(\chi) v) e_{j}, e_{j}\right) \cdot d \chi=\sum_{i \in I}\left(U(P v) e_{j}, e_{j}\right)=\Psi(U(P v))$

proving our assertion.-Note, that in particular, if $f \in \mathcal{B}_{S}^{+}$we have (cf. (a)) $\Psi(U(f))=$ $\Psi(U(f \mid D))$. Hence, by Lemma 5.2, we conclude, that

$$
\chi_{J}(\varphi)=\Phi(V(\varphi))=\int_{\mathfrak{B}} \Psi\left((h U)(\varphi \mid D) \cdot d h \quad(\leqslant+\infty) \quad\left(\varphi \in \mathcal{B}^{+}\right) .\right.
$$

(c) Let us put $\mathfrak{m}^{+}=\left\{v ; v \in \mathfrak{D}^{+}, 0 \leqslant \Psi(U(v))<+\infty\right\}$, and denote by $\mathfrak{R}$ the weak closure of all finite linear combinations of $\left\{U(v) ; v \in \mathfrak{m}^{+}\right\}$. We claim, that $\mathfrak{R}=R(U \mid D)$. In fact, let $P$ be the largest projection of $\Re$. Since $\Re$ is a two-sided ideal in $R(U \mid D)$, it is enough to show, that $P$ is the unit operator. We have evidently $s \cdot \mathrm{m}^{+} \subset \mathfrak{m}^{+}(s \in S)$ and thus $P$ belongs to the center of $R(U)$. Hence, by $U \in \mathrm{Fac}(S)$, it suffices to prove $P \neq 0$, or that $\mathrm{m}^{+}$contains an element $v \in \mathfrak{D}^{+}$such that $\Psi(U(v))>0$. By Remark 4.2.2 there is a $\varphi \in \mathcal{B}^{+}$with $0<\Psi(V(\varphi))<+\infty$ and therefore, by the last relation of (b) above, we can find a $h \in G$ satisfying $0<\Psi((h U)(\varphi \mid D))<+\infty$. Setting $v=h^{-1} \cdot(\varphi \mid D) \in \mathfrak{D}^{+}$, we obtain in this fashion, 
that $0<\Psi(U(v))<+\infty$. - Let us put $M=R(U \mid D)$ and $Z=\Psi \mid M$. From what we have just seen we conclude, that $Z$ is a semifinite trace on $M$.

(d) Since $D$ is invariant in $S$, there is a representation $s \mapsto \omega(s)$ of $S$ in $\operatorname{Aut}(M)$ such that $\omega(s)(A) \equiv U(s) A U\left(s^{-1}\right)(A \in M)$. Evidently $Z(\omega(s)(A)) \equiv Z(A)\left(A \in M^{+}\right)$. Observe, that also $\omega(s)(U(v)) \equiv U(s \cdot v)\left(v \in \mathfrak{D}^{+}\right)$. -We recall, that $U \mid D \approx W$ (cf. (b), Lemma 5.1). Putting $N=R(W)$, let $i: M \rightarrow N$ be a ${ }^{*}$ isomorphism such that $i(U(v)) \equiv W(v)(v \in D)$. There is a faithful, normal, semifinite trace $Z^{\prime}$ on $N^{+}$satisfying $Z^{\prime}(W(v)) \equiv Z(U(v))\left(v \in \mathfrak{D}^{+}\right)$. If we define $\omega^{\prime}(s) \in$ Aut $(N)$ by $\omega^{\prime}(s)(i(A)) \equiv i(\omega(s)(A)) \quad(A \in M)$, we have also $Z^{\prime}\left(\omega^{\prime}(s)(A)\right) \equiv$ $Z^{\prime}(A) \quad\left(s \in S ; A \in N^{+}\right)$.

(e) With the notations of (a), proof of Lemma 5.1, $W=\int_{A_{1}}^{\ominus} \sigma(x) \cdot d \mu(x)(\sigma(x) \in \operatorname{Irr}(D))$, where $\mathcal{A}_{1}$ is the closure of the image of $S$ in $\mathcal{A}$, and $\mu$ is an $S$ invariant Radon measure on $\mathcal{A}_{1}$. Since this decomposition of $W$ is central, we can write at the same time $Z^{\prime}=\int_{A_{1}}^{\oplus} Z_{x} \cdot d \mu(x)$, where $Z_{x}$ is faithful and normal on the full ring of the representation space of $\sigma(x)$ (cf. [5], Théorème 2, (iii), p. 200). By replacing $\mu$ through an equivalent measure $\mu^{\prime}$ we can achieve, that $Z_{x}$ has the standard normalization. In fact, let $e$ be an abelian projection in $N$, the central cover of which is equal to the unit operator. Writing $e=\int_{A_{1}}^{\oplus} e(x) \cdot d \mu(x)$, $a(x) \equiv Z_{x}(e(x))$ is $\mu$ measurable and positive almost everywhere; hence it suffices to define $\mu^{\prime}$ by $d \mu^{\prime}(x)=a(x) \cdot d \mu(x)$.- This being so we claim, that $\mu^{\prime}$ is a constant multiple of $\mu$. To see this it is enough to prove, that $\mu^{\prime}$ is $S$ invariant. This follows at once by observing, that for all $s \in S$ and $A \in N^{+}$, if $A=\int_{A_{1}}^{\oplus} A(x) \cdot d \mu^{\prime}(x)$, we have

$$
\int_{A_{1}} \operatorname{Tr}(A(x)) \cdot d \mu^{\prime}(x)=Z^{\prime}(A)=Z^{\prime}\left(\omega^{\prime}(s)(A)\right)=\int_{A_{1}} \operatorname{Tr}\left(A\left(s^{-1} \cdot x\right)\right) \cdot d \mu^{\prime}(x) .
$$

Summing up, assuming, that $\mu$ is suitably normalized, we have shown

$$
\Psi(U(\varphi \mid D))=Z^{\prime}(W(\varphi \mid D))=\int_{A_{1}} \operatorname{Tr}(\sigma(\varphi \mid D)) \cdot d \mu(\sigma) \leqslant+\infty \quad\left(\varphi \in \mathcal{B}^{+}\right) .
$$

(f) Let $h \in G$ and $p \in \mathscr{B}=G / S$ its canonical image. We write again (cf. loc. cit) $\mu_{p}$ for the measure on $\mathcal{A}$, which is the translate, carried by the coset $p$, of $\mu$. By what we have just seen, we conclude, that $\Psi((h U)(\varphi \mid D))=\int_{A} \operatorname{Tr}(\sigma(\varphi \mid D)) \cdot d \mu_{p}(\sigma)$. On the other hand, if $\lambda$ is an appropriately normalized $G$ invariant Radon measure on $\mathcal{A}$, we have $\lambda=\int_{\mathscr{H}} \mu_{p} \cdot d h(p)$ (cf. loc. cit.). Hence finally, by the end of (b) above

$$
\chi_{J}(\varphi)=\int_{\mathfrak{B}} \Psi((h U)(\varphi \mid D)) \cdot d h=\int_{\mathfrak{B}}\left(\int_{\mathcal{A}} \operatorname{Tr}(\sigma(\varphi \mid D)) \cdot d \mu_{p}(\sigma)\right) d h(p)=\int_{\mathcal{A}} \operatorname{Tr}(\sigma(\varphi \mid D)) \cdot d \lambda(\sigma),
$$

for all $\varphi \in B^{+}$, completing the proof of Lemma 5.3.

Q.e.d. 
We set $E=E(J) \in \hat{L} / \widetilde{G}, K=K(J)$ (cf. Notation 1.1.3) and form again $F=\{\varrho ; \varrho \in \hat{K}$, $\varrho \mid L \in E\}$ (cf. Lemma 1.1.5) and $F^{\prime}=\{\sigma ; \sigma \in \hat{D}, \sigma \mid L \approx \pi, \pi$ any in $E\}$ (cf. Lemma 4.1.4). We observe, that given $\sigma \in F^{\prime}$, there is a $\varrho \in F$ such that $\sigma \mid K \approx \varrho$. In fact, reasoning as in (a), proof of Lemma 1.2.1, we can write $\sigma \mid K=\int_{F}^{\oplus} \varrho^{\prime}(\zeta) \cdot d \tau(\zeta)$, where $\tau$ is quasi-invariant and ergodic with respect to $D$. But $D$ acts trivially on $F$ since $G_{Q} \equiv \mathfrak{U}(\varrho \in F)$ and $D \subset \mathfrak{U}$ (cf. the remarks before Lemma 4.1.2). Hence $\tau$ is concentrated in one point of $F$, proving our assertion. Since $\varrho$ is uniquely determined by $\sigma$, we can define $p: F^{\prime} \rightarrow F$ by $p(\sigma)=\varrho$ $(\sigma \mid K \approx \varrho)$.

LEMMA 5.4. With notations as above, the map $p: F^{\prime} \rightarrow F$ is $G$ equivariant, continuous and open.

Proof. The $G$ equivariance is clear.-As in (e), proof of Lemma 1.1.5, we denote by $\mathcal{F}$ the annihilator of $L$ in $X(K)$, and form the direct product $N=\widetilde{G} \times \mathcal{F}$. Let $\varrho$ be fix in $F$. We recall (cf. loc. cit.) that the map $i: N / N_{\varrho} \rightarrow F$ defined by $i\left(a N_{\varrho}\right)=a \varrho(a \in N)$ is a homeomorphism between $N / N_{\varrho}$ and $F \subset \hat{K}$.-As in the proof of Lemma 4.1.4, we write $\mathcal{I}^{\prime}$ for the annihilator of $L$ in $X(D)$ and set $N^{\prime}=\widetilde{G} \times \mathcal{F}^{\prime}$. Let $\sigma$ be fix in $F^{\prime}$. Then the map $i^{\prime}$ : $N^{\prime} \mid N_{\sigma}^{\prime} \rightarrow F^{\prime}$ defined by $i^{\prime}\left(a N_{\sigma}^{\prime}\right)=a \sigma\left(a \in N^{\prime}\right)$ is a homeomorphism between $N^{\prime} / N_{\sigma}^{\prime}$ and $F^{\prime} \subset \hat{D}$ (cf. loc. cit.). For $\varphi \in \mathcal{F}^{\prime}$ let us set $\gamma(\varphi)=\varphi \mid K \in \mathcal{F}$, and define the homomorphism $h: N^{\prime} \rightarrow N$ by $h((a, \varphi))=(a, \gamma(\varphi))$. Let $\sigma \in F^{\prime}$ be arbitrary and assume, that above $\varrho=p(\sigma)$. We put $A^{\prime}=N^{\prime} / N_{\sigma}, A=N / N_{\varrho} ;$ these are connected abelian Lie groups. If $a \in N^{\prime}$, we have $p(a \sigma)=$ $h(a) p(\sigma)=h(a) \varrho$, and thus there is a continuous surjective homomorphism $q: A^{\prime} \rightarrow A$, such that $q\left(a N_{\varrho}^{\prime}\right)=h(a) N_{Q}\left(a \in N^{\prime}\right)$. Hence $q$ is open. Observing, that the diagram

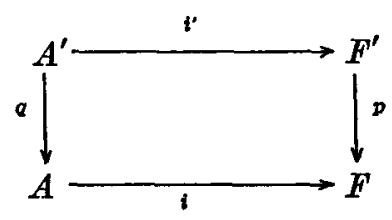

is commutative, we conclude finally, that $p$, too, is open.

Q.e.d.

We set $G_{1}=G(E)$ and write $M=K\left(G_{1}\right)_{0} \subset D$. $M$ is open in $D$; in fact, $M_{0}=D_{0}=\left(G_{1}\right)_{0}$. We denote by $\mathcal{E}$ the annihilator of $M$ in $X(D)$. We recall, that $G_{\varrho} \equiv \mathfrak{U}(\varrho \in F)$, and that $M \subset D \subset \mathfrak{u}$.

LEMMA 5.5. Let $\sigma$ be fix in $F^{\prime}$ and set $\varrho=p(\sigma)$. Then (i) The map $\varphi \mapsto \varphi \sigma(\varphi \in \mathcal{E})$ is a bijection between $\mathcal{E}$ and $p^{-1}(\varrho)$, (ii) $p^{-1}(\varrho)$ is the closure of $\mathfrak{U} \sigma$ in $F^{\prime}$. 
Proof. We shall use the notations of 4.1 assuming that $\pi$, at the start of loc.cit. is such, that $\sigma \mid L \approx \pi$.-We recall (cf. the proof of Lemma 4.1.4) that (1) Denoting by $\hat{E}^{\prime}$ the subset of $\hat{E}$ composed of all those elements, the restriction of which to $\mathcal{J}$ is a multiple of the conjugate of the identity map of $\mathcal{J}$ onto itself and, setting $f(\eta)=\left[\pi_{1}^{e} \otimes\left(\eta \circ \Phi_{1}\right)\right]^{-}\left(\eta \in \hat{E}^{\prime}\right.$; $\left.\pi_{1}^{e}=\pi^{e}\left|D, \Phi_{1}=\Phi\right| D^{e}\right)$, the map $\eta \mapsto f(\eta)$ is a bijection between $\hat{E}^{\prime}$ and the set of all $\sigma \in F^{\prime}$ with $\sigma \mid L \approx \pi$, (2) Writing $\mathcal{F}^{\prime \prime}$ for the annihilator of $\mathcal{J}$ in $X(E)$, there is an isomorphism $\tau: \mathcal{F}^{\prime} \rightarrow \mathfrak{I}^{\prime \prime}$ such that $\varphi f(\eta)=f(\tau(\varphi) \eta)\left(\eta \in \hat{E}^{\prime}\right)$. - Let us denote by $\mathcal{G}$ the collection of all those elements of $X\left(U^{*}\right)$, which on $\mathcal{T}$ restrict to the conjugate of the identity map $\mathfrak{J} \rightarrow \mathfrak{J}$. Since evidently $U^{\#} \subset E^{*}$, given $\eta \in \mathscr{E}^{\prime}$, there is a $\varphi \in \mathcal{G}$ with $\eta \mid U^{*} \approx \varphi$; we put $\varphi=\lambda(\eta)$. Setting $\pi_{2}^{e}=\pi^{e}\left|K, \Phi_{2}=\Phi\right| K^{e}$ and, for $\psi \in \mathcal{G}, g(\psi)=\left[\pi_{2}^{e}\left(\psi \circ \Phi_{2}\right)\right]^{\sim}\left([]^{\sim}\right.$ interpreted similarly as above), clearly the map $\psi \mapsto g(\psi)$ is an injection of $\mathcal{G}$ into $F$. Since $f(\eta) \mid K \approx g(\lambda(\eta))$, we have $p(f(\eta))=g(\lambda(\eta))$, or

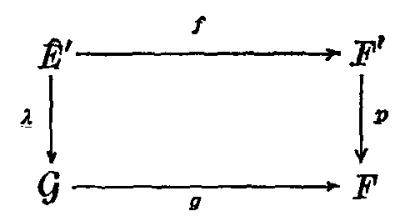

Let us denote by $\mathcal{E}_{1}$ the annihilator of $\Gamma_{0} U^{*}$ in $X(E)$; one sees at once, that $\tau(\mathcal{E})=\mathcal{E}_{1}$. We write, as in the proof of Lemma 4.1.1, $H=\left(\Gamma_{0}\right)^{*} \times \Delta$, and denote by $\Delta_{1}$ the subgroup of $\Delta$ such that $U^{*}=\left(\Gamma_{0}\right)^{*} \times \Delta_{1}$. Let $\mathcal{E}_{2}$ be the annihilator of $\Delta_{1}$ in $X(\Delta)$. The map $p \mapsto \varphi \mid \Delta$ $\left(\varphi \in \mathcal{E}_{1}\right)$ is an isomorphism from $\mathcal{E}_{1}$ onto $\mathcal{E}_{2}$. We conclude from this that, if $\tau \in \hat{E}^{\prime}$ and $\omega=$ $\lambda(\tau)$, the map $\varphi \mapsto \varphi \tau\left(\varphi \in \mathcal{E}_{1}\right)$ is a bijection between $\mathcal{E}_{1}$ and $\lambda^{-1}(\omega)$. In fact, by Lemma 4.1.1, $E=\Gamma_{0} \times \Delta$, and hence we can write $\tau$ as outer Kronecker product $\pi_{0} \times \chi\left(\pi_{0} \in \hat{\Gamma}_{0}, \chi \in X(\Delta)\right)$.This being said let us show, that $p^{-1}(\varrho)$ is an $\mathcal{E}$ orbit. In fact, if $\sigma^{\prime}, \sigma^{\prime \prime} \in F^{\prime}$ are such, that $p\left(\sigma^{\prime}\right)=p\left(\sigma^{\prime \prime}\right)=\underline{g}$ then, in particular, $\sigma^{\prime}\left|L \approx \pi, \sigma^{\prime \prime}\right| L \approx \pi$, and thus we can find $\eta^{\prime}, \eta^{\prime \prime} \in \hat{E}^{\prime}$ with $\sigma^{\prime}=f\left(\eta^{\prime}\right), \quad \sigma^{\prime \prime}=f\left(\eta^{\prime \prime}\right)$. Hence $g\left(\lambda\left(\eta^{\prime}\right)\right)=p\left(f\left(\eta^{\prime}\right)\right)=p\left(f\left(\eta^{\prime \prime}\right)\right)=g\left(\lambda\left(\eta^{\prime \prime}\right)\right.$ providing $\lambda\left(\eta^{\prime}\right)=$ $\lambda\left(\eta^{\prime \prime}\right)$. Let $\psi \in \mathcal{E}_{1}$ be such, that $\eta^{\prime \prime}=\psi \eta^{\prime}$, and assume $\psi=\tau(\varphi)(\varphi \in \mathcal{E})$. Then $\varphi \sigma^{\prime}=\varphi f\left(\eta^{\prime}\right)=$ $f\left(\tau(\varphi) \eta^{\prime}\right)=f\left(\eta^{\prime \prime}\right)=\sigma^{\prime \prime}$, proving our assertion. If $\varphi \sigma=\sigma$ for some $\varphi \in \mathcal{E}$, and $\sigma=f(\eta)$, we have $f(\tau(\varphi) \eta)=\varphi f(\eta)=\varphi \sigma=\sigma=f(\eta)$, whence $\tau(\varphi) \eta=\eta, \tau(\varphi)=1$ and $\varphi=1$. Summing up, we have completed to prove, that the map $\varphi \mapsto \varphi \sigma(\varphi \in \mathcal{E})$ is a bijection between $\mathcal{E}$ and $p^{-1}(\varrho)(\varrho=p(\varrho))$, which is statement (i) of our lemma.-We have clearly $\mathfrak{H}_{\sigma} \subset p^{-1}(\varrho)$ and, since $p: F^{\prime} \rightarrow F$ is continuous, also $\overline{\mathfrak{I} \sigma} \subset p^{-1}(\varrho)$. In this fashion, to establish (ii) it is enough to show that, always with the notations of 4.1 , putting $\vartheta=\Phi^{-1}\left(\Gamma_{1}\right) / \mathbf{T}$ we have $\bar{\vartheta} \sigma \supseteq p^{-1}(\varrho)$. Assume, that $\sigma=f(\eta)\left(\eta \in \hat{E}^{\prime}\right)$. By the proof of Lemma 4.1.3, if $a \in \vartheta$ and $\gamma=\Phi((a, 1))$, we have $a \sigma=$ $f(\gamma \eta)(\mathrm{A} .5)$. On the other hand, for each $\gamma \in \Gamma_{1}$ there is a $\varphi_{\gamma} \in \mathcal{E}_{1}$ such that $\gamma \eta=\varphi_{\gamma} \eta$. Hence, to complete our proof, it suffices to show, that $\left\{\varphi_{\gamma} ; \gamma \in \Gamma_{1}\right\}$ is dense in $\mathcal{E}_{1}$. If not, there is 
an $e \in E-\left(U^{*} \cdot \Gamma_{0}\right)$, satisfying $\varphi_{\gamma}(e) \equiv 1\left(\gamma \in \Gamma_{1}\right)$, which is equivalent to say, that $e$ commutes with $\Gamma_{1}$. Since $E=H \cdot \Gamma_{0}$, we can put $e=h \cdot \gamma_{0}\left(h \in H, \gamma_{0} \in \Gamma_{0}\right)$. Then $h$ commutes with $\Gamma_{1}$ and hence, by $H \subset \Gamma_{1}=C\left(\Gamma_{0}\right)$, also with $\Gamma_{0}$. In this fashion, $h$ is in the center of $\Gamma_{0} \cdot \Gamma_{1}=U$ and $e \in U^{\#} \cdot \Gamma_{0}$, giving a contradiction.

Q.e.d.

Remark 5.2. Using the previous lemma, one can easily show the following. Let $J \in \operatorname{Prim}(G)$ and $\mathcal{A} \in F^{\prime} / \Sigma^{\prime}$ be as e.g. in Lemma 5.3. Then $J$ is of type $I$ (cf. $\S 3$ ) if and only if $G$ acts transitively on $\mathcal{A}$. (One should compare this with Proposition 3 ).

For $\mathcal{A}(J)$ below, ef. Proposition 1; for $K(J)$, cf. Notation 1.1.3.

Theorem 2. Let $J$ be an arbitrary element of Prim $(G)$, If $\mu$ is a properly normalized $g$ invariant Radon measure on $\mathcal{A}(J)$ (cf. Lemma 2.3.5), we have

$$
\chi_{J}(\varphi)=\int_{A^{(J)}} \operatorname{Tr}\left(\varrho(\varphi \mid K(J)) \cdot d \mu(\varrho) \quad\left(\varphi \in \mathcal{B}^{+}\right) .\right.
$$

Proof. Below we shall write mostly $\mathcal{A}=\mathcal{A}(J), K=K(J)$; on the other hand we replace, in Lemma 5.3, $\mathcal{A}$ through $\mathcal{A}^{\prime}$. We recall, that by loc. cit.

$$
\chi_{J}(\varphi)=\int_{\mathcal{A}^{\prime}} \operatorname{Tr}(\sigma(\varphi \mid D)) \cdot d \lambda(\sigma) \quad\left(\varphi \in \mathcal{B}^{+}\right),
$$

where $\lambda$ is a $G$ invariant Radon measure on $\mathcal{A}^{\prime} \in F^{\prime} / \Sigma^{\prime}$ (cf. Lemma 4.1.5).

(a) We claim, that $p\left(\mathcal{A}^{\prime}\right)=\mathcal{A}(J)$. To this end let us show first, that $p\left(\mathcal{A}^{\prime}\right) \in F / \Sigma$ or, that $p\left(\mathcal{A}^{\prime}\right)$ is of the form $\overline{G_{\varrho}}$ (cf. Lemma 1.1.6). If $\sigma \in \mathcal{A}^{\prime}$, we have $\mathcal{A}^{\prime}=\overline{G \sigma}$; hence, putting $\varrho=p(\sigma), \overline{G_{\varrho}} \supset p\left(\mathcal{A}^{\prime}\right)$. On the other hand we conclude from Lemma 5.5, that $\mathcal{A}^{\prime}$ is the complete inverse image of $p\left(A^{\prime}\right)$. Since, by Lemma 5.4, $p$ is an open map $F^{\prime} \rightarrow F$, proving $\overline{G_{\varrho}}=p\left(\mathcal{A}^{\prime}\right)$, and hence $p\left(\mathcal{A}^{\prime}\right) \in F / \Sigma$. To show, that $p(\mathcal{A})=\mathcal{A}(J)$ we recall (cf. the proof of Lemma 4.1.4), that if $V \in \mathrm{Fac}(G)$ satisfies $\operatorname{ker}(V)=J$, then $V \mid D=\int_{A^{\prime}}^{\oplus} \sigma^{\prime}(x) \cdot d \tau(x)\left(\sigma^{\prime}(x) \approx x\right)$, and thus $V \mid K(J)=\int_{p\left(A^{\prime}\right)}^{\oplus} \varrho^{\prime}(y) \cdot d \xi(y)\left(\varrho^{\prime}(y) \approx y\right)$, whence the desired conclusion follows by Proposition 1, (iii).

(b) Let $\mathcal{E}$ and $M$ be as in Lemma 5.5. We denote by $d \psi$ the normalized Haar measure on $\mathcal{E}$ and observe, that there is a $G$ invariant Radon measure $\mu$ on $\mathcal{A}=\mathcal{A}(J)$ such that, for all $\left.f \in \mathcal{L}\left(\mathcal{A}^{\prime}\right), \int_{A^{\prime}} f(\sigma) \cdot d \lambda(\sigma)=\int_{A}\left(\int_{\varepsilon} f(\psi \sigma) \cdot d \psi\right)\right) d \mu(\varrho)$. Reasoning, as in (b), proof of Lemma 5.3, we note, that $\int_{\varepsilon} \operatorname{Tr}((\psi \sigma)(\varphi \mid D)) \cdot d \psi=\operatorname{Tr}(\sigma(\varphi \mid M))$. Hence we conclude that $\chi_{J}(\varphi)=$ $\int_{A} \operatorname{Tr}(\beta(\varphi \mid M)) \cdot d \mu(\varrho)\left(\varphi \in \mathcal{B}^{+}\right)$where, for each $\varrho \in \mathcal{A}, \beta \in \hat{M}$ is subject to the condition, that $\beta \mid K \approx \varrho .-W i t h$ the notations as just employed, we shall have completed the proof of our theorem by showing, that $\operatorname{Tr}(\beta(\varphi \mid M)) \equiv \operatorname{Tr}(\varrho(\varphi \mid K))\left(\varphi \in \mathcal{B}^{+}\right)$.

(c) We claim, that there is a closed subgroup $M_{1}$ of $G$, with $K \subset M_{1} \subset M$, and an 
$\alpha \in \hat{M}_{1}$ such that (i) $\beta=$ ind $_{M_{1} \uparrow M} \alpha$, (ii) If $\mathfrak{H}$ is the annihilator of $K$ in $X\left(M_{1}\right), p \alpha=\chi_{p} \alpha(p \in M)$ and the map $p \mapsto \chi_{\mathfrak{p}}$ gives rise to an isomorphism between $M / M_{1}$ and $\mathfrak{5}$. In fact (1) With notations as in 4.1, let us put $A=U^{*} \cdot \Gamma_{0}, \pi_{3}^{e}=\pi^{e}\left|M, \Phi_{3}=\Phi\right| M^{e}$. We have then $M=$ $\Phi^{-1}(A) / \mathbf{T}$, and analogously as at loc. cit., with an appropriately chosen $\tau \in \hat{A}, \beta=$ $\left[\pi_{3}^{e} \otimes\left(\tau \circ \Phi_{3}\right)\right]^{\sim}$. (2) $A$ is of the form $\Gamma_{0} \times \Lambda$, where $\Lambda$ is discrete abelian, and $\Gamma_{0}$ is central extension of a vector group with the circle group. Therefore, if $A_{1}$ is a maximal abelian subgroup of $A$, there is a $\eta \in X\left(A_{1}\right)$ such that $\tau=\operatorname{ind}_{A_{4} \uparrow A} \eta$. We have furthermore for any $\gamma \in A, \gamma \tau=\varphi_{\gamma} \tau$, and the map $\gamma \mapsto \varphi_{\gamma}$ gives rise to an isomorphism between $A / A_{1}$ and the annihilator of $A_{1}$ in $X(A)$. (3) We recall, that if $H$ is any locally compact group, $H_{1}$ a closed subgroup of $H$ and $V_{1} \in \operatorname{Rep}\left(H_{1}\right), V \in \operatorname{Rep}(H)$, then $\left(\operatorname{ind}_{H_{1} \uparrow H} V_{1}\right) \otimes V=$ $\operatorname{ind}_{H_{1} \uparrow H}\left(V_{1} \otimes\left(V \mid H_{1}\right)\right)$. Therefore putting $M_{1}=\Phi^{-1}\left(A_{1}\right) / \mathbf{T}, \pi_{4}^{e}=\pi^{e}\left|M_{1}, \quad \Phi_{4}=\Phi\right| M^{e}$ and $\alpha=\left[x_{4}^{e} \otimes\left(\eta \circ \Phi_{4}\right)\right]^{-}$, we obtain $\beta=\operatorname{ind}_{M_{1} \uparrow M} \alpha$, proving (i). (4) As in the proof of Lemma 4.1.3 we see, that if $a \in M_{1}$ and $\gamma=\Phi((a, 1)) \in A_{1}$, then $a \alpha=\left[\pi_{4}^{e} \otimes\left(\gamma \eta \circ \Phi_{4}\right)\right]^{2}$. From this, by what we saw in (2), we conclude that, for $p \in M, p \alpha=\chi_{p} \alpha$, and the map $p \mapsto \chi_{p}$ gives rise to an isomorphism between $M / M_{1}$ and $\mathfrak{S} \subset X(M)$, proving (ii).

(d) Let us put $f=\varphi \mid M \in \mathcal{B}_{M}^{+}$. By Remark 5.1, if $d v$ is a suitably normalizedinvariant measure on $M / M_{1}$, we have

$$
\operatorname{Tr}(\beta(f))=\int_{M / M_{1}} \operatorname{Tr}\left((p \alpha)\left(f \mid M_{1}\right)\right) \cdot d v(p)
$$

Therefore, denoting by $d \psi$ the image of $d v$ on $\mathfrak{H}$, we obtain

$$
\operatorname{Tr}(\beta(f))=\int_{f} \operatorname{Tr}\left((\psi \alpha)\left(f \mid M_{1}\right)\right) \cdot d \psi .
$$

(e) We set $g=f \mid M \in \mathcal{B}_{M}^{+}$. By Remark 2.1.2 if $d w$ is a properly chosen invariant measure on $M_{1} / L, \alpha(g)=\int_{M_{1} / L} \alpha(g(x)) \alpha(x) \cdot d w(x)$. To simplify notations in the following, given $\psi \in \mathfrak{S}\left(\subset X\left(M_{1}\right)\right)$ we denote in the same fashion the corresponding element of $X\left(M_{1} / K\right)$. Let $d u$ be the measure, dual to $d \psi$, on $M_{1} / K$, and let $d y$ be the invariant measure on $K / L$ satisfying $d w=d u \cdot d y$. If $e$ is a fixed element of $H(\alpha)$, the function $G(u)=\int_{K_{L} L}(\alpha(g(x y)) \alpha(x y) e, e) \cdot d y$ is continuous and of a compact support on $M_{1} / K$, and we have

Therefore

$$
((\psi \alpha)(g) e, e)=\int_{M_{\mathfrak{1} / K}} \psi(u) G(u) \cdot d u \geqslant 0 \quad(\psi \in \mathfrak{S}) .
$$

$$
\int_{\mathfrak{G}}((\psi \alpha)(g) e, e) \cdot d \psi=\int_{\mathfrak{v}}\left(\int_{M_{1} / Z} \psi(u) G(u) \cdot d u\right) \cdot d \psi=\int_{K^{\prime} / L}(\alpha(g(y)) \alpha(y) e, e) \cdot d y=(\alpha(g \mid K) e, e) .
$$


(f) Let $\left\{e_{j}\right\}$ be a complece orthonormal system in $H(\alpha)$. Given, that $g=f \mid M_{1}$, we obtain by virtue of (e) from the last equation of (d)

$$
\operatorname{Tr}(\beta(f))=\sum_{j} \int_{g}\left((\psi \alpha)(g) e_{j}, e_{j}\right) \cdot d \psi=\sum_{j}\left(\alpha(f \mid K) e_{j}, e_{j}\right)=\operatorname{Tr}(\varrho(f \mid K))
$$

since clearly $\alpha \mid K=Q$.- Substituting $f=\varphi \mid M\left(\varphi \in B^{+}\right)$we obtain the last relation of (b), and hence the proof of Theorem 2 is complete.

Q.e.d.

\section{§ 6}

In this last section we shall assume, that $G$ is a connected, simply connected and solvable Lie group with the Lie algebra $\mathfrak{g}$. For more about the background of the following summary ef. [24], Introduction, p. 74 and 3, p. 80.-Given $g \in \mathfrak{g}^{\prime}$ (=dual of the underlying space of $\mathfrak{g}$ ) we denote by $\bar{G}_{g}$ the reduced stabilizer of $g$ (cf. loc. cit. 3.3.a), p. 83). Putting $\left(G_{g}\right)_{0}=\exp \left(\mathfrak{g}_{g}\right)$ (A.1, A.10) there is a $\chi_{g} \in X\left(\left(G_{g}\right)_{0}\right)$ such that $d \chi_{g}=i\left(g \mid \mathfrak{g}_{g}\right)$. We set $\stackrel{A}{G_{g}}=\left\{\chi ; \chi \in X\left(\bar{G}_{g}\right), \chi \mid\left(G_{g}\right)_{0} \equiv \chi_{g}\right\}, R=\cup_{g \in g} \cdot \stackrel{A}{G}_{g}$ and recall (cf. loc. cit. 3.6, p. 84), that one can define an equivalence relation $\subseteq$ on $R$ as follows. Let $\tilde{G}$ and $L=[\tilde{G}, \tilde{G}]=(G, G]$ be as in I.1. Writing $L=\exp (\mathfrak{D})$, we have $\mathfrak{D}=[\mathfrak{g}, \mathfrak{g}]$. Given $E \in \hat{L} / \tilde{G}$, we denote by $\Omega$ its complete inverse image, via the Kirillov isomorphism between $\delta^{\prime} / L$ and $\hat{L}$ (loc. cit. 3.1.a)), in $g^{\prime}$. We have $K(E)=\bar{G}_{g} L$ (cf. Notation I.1.1), where $g$ is arbitrary in $\Omega$. Putting $K=K(E)$ we set $\stackrel{\ominus}{K}=\left\{\chi ; \chi \in X(K), \chi \mid K_{0} \equiv 1\right\}$. Then $\mathfrak{B}(\Omega)=\bigcup_{g \in \Omega} \stackrel{A}{G}_{g}$ can be endowed with the structure of a bundle over $\Omega$, with $\stackrel{\ominus}{K}$ as structure group (loc. cit. 3.5.a), p. 84). This being so there is an equivalence relation $\subseteq$ on $R$, such that $p_{1} \equiv p_{2}(\Im)$ if and only if there is an $E \in \hat{L} / \tilde{G}$ with $\Omega$ (as above) containing both $p_{1}$ and $p_{2}$ and $\overline{G p_{1}}=\overline{G p_{2}}$, the closure being taken in $\mathfrak{B}(\Omega)$.-We put $\mathbf{S}=\boldsymbol{R} / \mathfrak{S}$ and call its elements the generalized orbits of G.-Given $O \in \Theta$, we can associate with it a unitary equivalence class $F(O)$ of factor representations of $G$ in the following steps. Assume, that again $\mathfrak{U}=\bigcup_{E \in \hat{L} / \tilde{G}} F(E)$ (cf. Lemma 1.1.6). (1) We recall (loc. cit. 3.3.c), p. 83), that there is a map $\lambda: R \rightarrow \mathfrak{A}$ such that $\lambda(p) \mid L=\varepsilon(p)(p \in \mathbb{R})$ where, if $p=(g, \chi), \varepsilon(p)$ is the image of $g \mid \delta$ in $\hat{L}$, and $F(p)=\operatorname{ind}_{K \uparrow G} \lambda(p) \in \operatorname{Fac}(G)(K=K(E)$, if $p \in \Omega$ as above) is unitarily equivalent to a holomorphically induced monomial representation (that is a unitary representation of the form ind $(\mathfrak{h}, p)$; cf. [22], Theorem 1 , p.512) (2) There is an, essentially uniquely determined positive $G$ invariant Radon measure $d v$ on $O$ (cf. [24], 3.6, p. 85), (3) If $p \mapsto T(p) \in F(p)(p \in O)$ is Borel measurable, $F(O)$ is defined as the unitary equivalence class of $\int_{O}^{\oplus} T(p) \cdot d v(p)$.- Let us define the map $l: \mathrm{S} \rightarrow \hat{G}$ by $l(O)=$ quasi-equivalence class of $F(O)(O \in \mathbf{S})$, and let us set $J(O)=\operatorname{ker}(l(O))$. The principal result of [24] (cf. Theorem 1, p. 114) states, that $O \rightarrow J(O)$ is a bijection between $\mathbf{S}$ and Prim $(G)$. Below (cf. Theorem 3) we show, that $O \rightarrow l(O)$ is a bijection between $\mathbf{S}$ 
and $\hat{G}_{\text {norm }}$ (for the latter cf. 4.2) whence the previous result, by virtue of Theorem 1 , follows easily. In fact, let us put $\delta(l)=\operatorname{ker}(l)(l \in \hat{G})$; we have

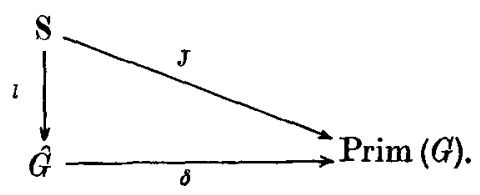

To obtain the stated conclusion, it suffices to recall, that by loc. cit. $\delta \mid \hat{G}_{\text {norm }}$ is a bijection between $\hat{G}_{\text {norm }}$ and Prim $(G)$.

THEOREM 3. With notations as above, the map $l: \mathbf{S} \rightarrow \hat{G}$ is a bijection between the set $\mathbf{S}$ of all generalized orbits and $\stackrel{n}{\text { norm }}_{\text {. }}$.

Proof. Let us choose a primitive ideal $J \in \operatorname{Prim}(G)$. Writing $K=K(J)$ (cf. Notation 1.1.3) and $\mathcal{A}=\mathcal{A}(J) \subset \hat{K}$ (cf. Proposition 1) we recall (cf. Lemma 2.3.6), that if $\varrho: \mathcal{A} \rightarrow \operatorname{Irr}(K)$ ) is a Borel cross section and $\mu$ a $G$ invariant Radon measure on $\mathcal{A}$ (cf. Lemma 2.3.5), then

$$
T=\operatorname{ind}_{K \uparrow G}\left(\int_{A}^{\oplus} \varrho(\xi) \cdot d \mu(\xi)\right) \quad(\in \operatorname{Fac}(G))
$$

is a normal representation of kernel $J$. We are going to construct an $O \in \mathrm{S}$, such that $T$ belongs to the quasi-equivalence class $l(\mathcal{O})$, as follows. We set $E=E(J) \in \hat{L} / \tilde{G}$ (cf. Lemma 1.1.8) and form the corresponding $\Omega \subset g^{\prime}$ as above. Putting $F=F(E)$ (cf. Notation 1.1.2) and writing $\lambda$ for $\lambda \mid \mathfrak{B}(\Omega)$ we recall that (1) $\lambda$ is a continuous, open map from $\mathfrak{B}(\Omega)$ onto $F$ (cf. [24], Lemma 6, p. 91), (2) We set $G_{1}=G(E)$ (cf. Notation 1.1.1) and form, as in Lemma 5.5, $M=K\left(G_{1}\right)_{0}$. Then, for $g \in \Omega$ arbitrary and $f=g \mid \mathfrak{D}$, we have $M=\bar{G}_{g}\left(G_{f}\right)_{0} L$, and $\lambda\left(p_{1}\right)=$ $\lambda\left(p_{2}\right)$ if and only if $M p_{1}=M p_{2},(3) \lambda$ is equivariant with respect to $\tilde{G} \times \stackrel{\ominus}{K}$ (loc. cit. 3.3.c), p. 82)-We show next, that $\lambda$ gives rise to a bijection between $\mathfrak{B}(\Omega) / \varsigma$ and $F / \Sigma$ (cf. Lemma 1.1.6). To this end, it is enough to see, that $\lambda(\overline{G p)}=\overline{G \lambda(p)}$. But since $\overline{G p}$ is $M$ invariant, and $\lambda$ open, we have $\lambda(\overline{G p)} \supset \overline{G \lambda(p)}$, whence the stated conclusion is clear.-Let $\mathcal{O} \in \mathrm{S}$ be such, that $\lambda(\mathcal{O})=\mathcal{A}$ (as above), and $d v$ a positive, $G$ invariant Radon measure on $O$. If $v^{*}$ is a finite measure equivalent to $v$, then $\lambda_{*}\left(v^{*}\right)$ is equivalent to $\mu$. In fact, there is a closed, connected subgroup $\mathcal{G} \subset \tilde{G} \times \stackrel{\ominus}{K}$, such that $M \subset \mathcal{G}$ and $O=\mathcal{G p}$ (cf. [22], (d) in the proof of Proposition 7.1, p. 541). Moreover, $O$ is homeomorphic to $\mathcal{G} / \mathcal{G}_{p}$ and $v$ is image of a $\mathcal{G}$ invariant measure on $\mathcal{G} / \mathcal{G}_{p}$ (cf. loc. cit. Proposition 1.1, p. 515). Hence it suffices to observe, that $\mathcal{A}$ is homeomorphic to $\mathcal{G} / \mathcal{G}_{p} M$ and $\mu$ is the image of a $\mathcal{G}$ invariant meas. ure--Observe, that from what we have just seen we can conclude, that $v=\int_{A} v_{\xi} \cdot d \mu(\zeta)$, where $v_{\zeta}$ is carried by $\lambda^{-1}(\zeta)(\zeta \in A)$. Let $T$ be as at the start of our proof. Setting $Z(\zeta)=$ 
$\operatorname{ind}_{K \uparrow G} \varrho(\zeta)(\zeta \in \mathcal{A})$ we have $T=\int_{A}^{\oplus} Z(\zeta) \cdot d \mu(\zeta)$. Let us put $T(p)=Z(\lambda(p))(p \in O)$; observe, that $T(p) \in F(p)$, and therefore $T^{\prime}=\int_{O}^{\oplus} T(p) \cdot d v(p)$ belongs to $F(O)$. We claim, that $T^{\prime} \approx T$. In fact, since $\int_{0}^{\oplus} T(p) \cdot d v_{\zeta}(p) \approx Z(\zeta)$, by $v=\int_{A} v_{\zeta} \cdot d \mu(\zeta)$ we obtain (cf. [17], Theorem 2.11, p. 204)

$$
T^{\prime}=\int_{0}^{\oplus} T(p) \cdot d v(p)=\int_{A}^{\oplus}\left(\int_{0}^{\oplus} T(p) \cdot d v_{\zeta}(p)\right) d \mu(\zeta) \approx \int_{A}^{\oplus} Z(\zeta) \cdot d \mu(\zeta)=T
$$

proving our assertion. Summing up, given $J \in \operatorname{Prim}(G)$, and $T \in \mathrm{Facn}(G)$ with $\operatorname{ker}(T)=J$, we have found an $O \in \mathbf{S}$, such that the quasi-equivalence class of $T$ is equal to $l(O)$. Since, by Proposition 4 , the assumptions $T_{1} \in \mathrm{Facn}(G)$ and $\operatorname{ker}\left(T_{1}\right)=J$ imply $T_{1} \approx T$, we can conclude, that $l(\mathbf{S}) \supset \hat{G}_{\text {norm }}$.-If, in our previous reasoning, in place of $\mathcal{A} \in \mathfrak{A} / \Sigma$ we start with $O \in \mathbf{S}$, we shall obtain, that $l(O) \in \hat{G}_{\text {norm }}$, and hence $l(\mathbf{S})=\hat{G}_{\text {norm }}$-In this fashion, to complete the proof of our theorem, it suffices to show, that $l\left(O_{1}\right)=l\left(O_{2}\right) \mathrm{im}$. plies $O_{1}=O_{2}$. Let us put $\lambda\left(O_{k}\right)=\mathcal{A}_{k} \in \mathfrak{Q} / \Sigma$, and suppose, that $\mathcal{A}_{k}=\mathcal{A}\left(J_{k}\right)\left(J_{k} \in \operatorname{Prim}(G)\right.$, $k=1,2)$. If $T_{k} \in$ Facn $(G)$ is such, that $\operatorname{ker}\left(T_{k}\right)=J_{k}(k=1,2)$ then, by what we saw above, $T_{k}$ is of the quasi-equivalence class $l\left(O_{k}\right)(k=1,2)$. Therefore, by assumption, $T_{1} \approx T_{2}$, and hence $J_{1}=\operatorname{ker}\left(T_{1}\right)=\operatorname{ker}\left(T_{2}\right)=J_{2}$, and thus also $\mathcal{A}_{1}=\mathcal{A}_{2}$ and $O_{1}=O_{2}$. Q.e.d.

\section{Appendix: Some general assumptions and notational conventions}

(1) Given a Lie algebra $g$ (defined always over the real field and finite dimensional), $\exp (\mathfrak{g})$ will denote a connected and simply connected Lie group with the Lie algebra $\mathfrak{g}$. If $G=\exp (\mathfrak{g})$ and $\mathfrak{h}$ is some subalgebra of $\mathfrak{g}$, exp (h) will stand for the connected subgroup, determined by $\mathfrak{h}$, of $G$.

(2) All unitary spaces considered are assumed separable. If $W$ is a unitary representation of a group or a * representation of a $C^{*}$ algebra, $H(W)$ denotes its representation space and $R(W)$ the weak closure of the collection of all linear combinations with complex coefficients of operators of $W$.-When quoting the literature, we shall often leave to the reader to translate results concerning representations of $C^{*}$ algebras in the group representation context.

(3) If $W$ is a * representation of a $C^{*}$ algebra, it will be assumed to be nondegenerate, and thus $R(W)$ always contains the unit operator.

(4) All locally compact groups and $C^{*}$ algebras are supposed to be separable. If $G$ is such a group or $A$ is a $C^{*}$ algebra, we shall write $\operatorname{Rep}(G), \operatorname{Irr}(G)$ and Fac $(G)(\operatorname{Rep}(A)$, $\operatorname{Irr}(A)$ and $\operatorname{Fac}(A)$ resp.) for the set of all conerete representations, irreducible representations and factor representations resp.-The few exceptions, when we use these symbols in the sense of [4] to designate the related Borel spaces, will be specially noted. 
(5) Whenever convenient, we shall use the same symbol for a concrete representation and its unitary equivalence class. Thus if $T_{1}$ and $T_{2}$ are concrete representations, $T_{1}=T_{2}$ indicates, in general, only their unitary equivalence (unless specified otherwise by the context). Should the necessity arise to emphasize, that we are claiming only unitary equivalence, we shall write $T_{1} \sim T_{2}$.-Quasi-equivalence of $T_{1}$ and of $T_{2}$ will be denoted by $T_{1} \approx T_{2}$.-Analogous conventions will be observed for * representations of $C^{*}$ algebras.

(6) If $G$ is a group and $H$ an invariant subgroup of $G$, given $\pi \in \operatorname{Rep}(H)$ (cf. (4) above) and $a \in G, a \pi$ stands for the representation of $H$ defined by $(a \pi)(h) \equiv \pi\left(a^{-1} h a\right)(h \in H)$.

(7) If $T$ is a locally compact space, $\mathcal{L}(T)$ denotes the family of all continuous complexvalued functions with a compact support on $T$.

(8) Given a group $G$, we write $X(G)$ for the group of all continuous homomorphisms of $G$ into the circle group.-For $\pi \in \operatorname{Rep}(G)$ and $\chi \in X(G)$, $\chi \pi$ stands for the tensor product of $\pi$ with the one dimensional representation $\chi$.

(9) If the group $G$ acts on the set $X$ as a transformation group, given $p \in X$, we shall denote by $G_{p}$ or by $\operatorname{Stab}_{p}(G)$ the stabilizer of $p$ in $G$.

(10) Given a topological group $G$, we write $G_{0}$ for the connected component of the identity.

(11) We shall often use the following relation. Assume, that $G$ is a locally compact separable group, $K$ a closed invariant subgroup of $G$ and $T \in \operatorname{Rep}(K)$. Then, in the sense of unitary equivalence

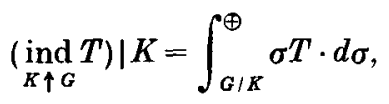

where $d \sigma$ is a Haar measure on $G / K, a: G / K \rightarrow G$ an arbitrary Borel cross section and $(\sigma T)(k) \equiv T\left((a(\sigma))^{-1} k a(\sigma)\right)(\sigma \in G / K)$.

(12) Given a group $G, G^{*}$ denotes its center. Same for algebras.

(13) Given a Hilbert space $H, B(H)$ stands for the ring of all bounded operators and $C(H)$ for the collection of all completely continuous operators.

(14) We use always the same symbol for a unitary representation of a group and for the corresponding representation of the group $C^{*}$ algebra.

(15) For a unitary representation $U, \bar{U}$ denotes its conjugate.

\section{References}

[1]. Auslander, L. \& Kostant, B., Polarization and unitary representations of solvable groups. Invent. Math., 14 (1971), 255-354.

[2]. Chevaldey, C., Théorie des groupes de Lie, vol. II, Aroupes algébriques. Act. Sci. Ind., $n^{\circ} 1152$, Hermann, Paris, 1951. 
[3]. Dixmier, J., Sur les C* algèbres. Bull. Soc. Math. France, 88 (1960), 95-112.

[4]. - Les $C^{*}$ algèbres et leurs représentations. Gauthier-Villars, Paris, 1964.

[5]. - Les algèbres d'opérateurs dans l'espace hilbertien, $2^{\text {nd }}$ edition. Gauthier-Villars, Paris, 1969.

[6]. - Sur la représentation régulière d'un groupe localement compact connexe. Ann. Sci. École Norm. Sup., 2 (1969), 423-436.

[7]. Duflo, M., Caractères des groupes et des algèbres de Lie résolubles, Ann. Sci. École Norm. Sup., 3 (1970), 23-74.

[8]. Efrros, E. G., A decomposition theory for representations of $\mathrm{C}^{*}$ algebras. Trans. Amer. Math. Soc., 107 (1963), 83-106.

[9]. Fell, J. M. G., The structure of algebras of operator fields. Acta Math., 106 (1961), 233280.

[10]. GLImm, J., Locally compact transformation groups. Trans. Amer. Math. Soc., 101 (1961), $124-138$.

[11]. Godfment, R., Sur la théorie des représentations unitaires. Ann. of Math., 53 (1951), 68-124.

[12]. - - Mémoire sur la théorie des caractères dans les groupes localement compacts unimodulaires. J. Math. Pures Appl., 30 (1951), 1-110.

[13]. - Théorie des caractères II. Définition et propriétés générales des caractères. Ann. of Math., 59 (1954), 63-85.

[14]. Guichardet, A., Caractères des algèbres de Banach involutives. Ann. Inst. Fourier, 13 (1962), I-81.

[15]. HalPERn, H., Quasi-equivalence classes of normal representations for a separable C* algebra. To appear.

[16]. Mackey, G. W., Induced representations of locally compact groups I. Ann. of Math., 55 (1952), 101-139.

[17]. — Induced representations of locally compact groups II. Ann. of Math., 58 (1953), 193-222.

[18]. —- Borel structure in groups and their duals. Trans. Amer. Math. Soc., 85 (1957), 134165.

[19]. — Unitary representations of group extensions. Acta Math., 99 (1958), 265-311.

[20]. - - Induced representations and normal subgroups, Proc. of the International Symposium on linear spaces, Jerusalem, 1960, 319-326.

[21]. Pukanszky, L., Characters of algebraic solvable groups. J. Functional Analysis, 3 (1969), 435-491.

[22]. - Unitary representations of solvable Lie groups, Ann. Sci. École Norm. Sup., 4 (1971), $457-608$.

[23]. - Action of algebraic groups of automorphisms on the dual of a class of type I groups. Ann. Sci. École Norm. Sup., 5 (1972), 379-396.

[24]. - The primitive ideal space of solvable Lie groups. Invent. Math., 22 (1973), 74-118.

[25]. - Characters of connected Lie groups. To appear in the Bull. Amer. Math. Soc. 